\title{
A Diagrammatic Notation for Visualizing Epistemic Entities and Relations
}

\author{
Kye Palider \\ Department of Philosophy, \\ University of Toronto \\ kye.palider@mail.utoronto.ca \\ Julia Da Silva \\ Department of English Language \& Literatures, \\ University of British Columbia \\ julia777@student.ubc.ca \\ Jessica Rapson \\ Munk School, \\ University of Toronto \\ jess.rapson@mail.utoronto.ca

\section{Yifang Zhang} \\ Department of Computer Science, \\ University of Toronto \\ jerus.zhang@mail.utoronto.ca
}

\author{
Paul Patton \\ IHPST, \\ University of Toronto \\ paul.patton@mail.utoronto.ca \\ Torin Doppelt \\ Independent Scholar \\ torin.doppelt@gmail.com \\ Ameer Sarwar \\ Neuroscience, \\ University of Oxford \\ ameer.sarwar@linacre.ox.ac.uk
}

Amna Zulfiqar

Department of Medicine,

University of Toronto

amna.zulfiqar@mail.utoronto.ca

\author{
Hakob Barseghyan \\ Victoria College, \\ University of Toronto \\ hakob.barseghyan@utoronto.ca \\ Nichole Levesley \\ IHPST, \\ University of Toronto \\ nichole.levesley@mail.utoronto.ca \\ Jamie Shaw \\ IHPST, \\ University of Toronto \\ jamieco.shaw@utoronto.ca
}

\section{Keywords}

diagrammatic notation, knowledge visualization, synchronic diagram, diachronic diagram, epistemic element, epistemic stance, epistemic agent, epistemic entity, epistemic relation

\begin{abstract}
This paper presents a diagrammatic notation for visualizing epistemic entities and relations. The notation was created during the Visualizing Worldviews project funded by the University of Toronto's Jackman Humanities Institute and has been further developed by the scholars participating in the university's Research Opportunity Program. Since any systematic diagrammatic notation should be based on a solid ontology of the respective domain, we first outline the current state of the scientonomic ontology. We then proceed to providing diagrammatic tools for visualizing the epistemic entities and relations of this ontology. These basic diagramming techniques allow us to construct diagrams of various types for both synchronic and diachronic visualizations. The paper concludes by highlighting some future research directions. As the notation presented here is de facto accepted and used in scientonomy, the paper suggests no modifications.
\end{abstract}




\section{Introduction}

Scientonomic scholarship has heavily relied on diagrams since the inception of the community. The diagramming techniques of The Laws of Scientific Change (Barseghyan, 2015) have been used in virtually every paper published by scientonomy community. Yet, until 2019 these diagramming techniques had not been systematically studied nor their pros and cons formally discussed. The time was then ripe to scrutinize these diagramming techniques in a systematic fashion and develop them into a full-fledged diagrammatic notation that would be capable of visualizing any epistemic entity or relation. These techniques were discussed in detail, analyzed, further developed, and systematized into a proper diagrammatic notation in May 2019, during the Visualizing Worldviews project funded by Jackman Humanities Institute as part of their Scholars-in-Residence program - a program that provides advanced undergraduate students at the University of Toronto with an intensive research opportunity. The notation was a product of the collaborative effort of an interdisciplinary team of researchers that included Hakob Barseghyan, Julia Da Silva, Torin Doppelt, Nichole Levesley, Kye Palider, Paul Patton, Jessica Rapson, Ameer Sarwar, Jamie Shaw, Yifang Zhang, and Amna Zulfiqar. The team worked intensely for the whole month of May 2019 on developing the notation and applying it to a wide range of belief systems from various fields of inquiry and time periods, including medieval Islamic medicine, modern European magic, late-eighteenth century French chemistry, nineteenth century views on localization of function in the cerebral cortex, twentieth century Aryan physics, and twentieth century scientific methodologies.

This notation has seen further development and use in scholarship since May of 2019. In the 2020 online edition of the Scholars-in-Residence program, Ori Gilboa, Faizan Malik, Lucie Perillat, Cameron Scott, Nicholas Slawnych, and Kevin Zhu joined Jamie Shaw, Paul Patton, Kye Palider, and Hakob Barseghyan in applying the notation to some high-profile present-day debates on intelligent design, gender, climate change, and race. Since Fall 2019, the notation has been elaborated by the scholars participating in the University of Toronto's Research Opportunity Program. Cyrus Al-Zayadi, Josh Allen, Zoë Golay, Carlin Henikoff, Nichole Levesley, Tessa Ng, Alexander Offord, Sanghoon Oh, Cameron Scott, and Neo Yin worked with Kye Palider, Paul Patton, and Hakob Barseghyan on applying the notation to a broad variety of topics from ancient, medieval, and early modern science. There projects explored many aspects of the Aristotelian-medieval optics, physics, cosmology, alchemy, astrology, ethics, and theology, among other fields. The diagrammatic notation has displayed its flexibility in its application to a variety of belief systems and debates throughout history. By extracting various aspects of these belief systems and debates, these applications clearly illustrate the logical relations between concepts, showcase flaws and/or ambiguities in arguments, aid in the identification of important agents in the given community, and facilitate the comparison and contrasting of opposing views. Among other things, these efforts highlight the advantages of diagrammatic representation over pure text in locating gaps and inconsistencies in the historical record. They show how a relatively basic notation can produce diagrams with a variety of purposes and specialties.

The purpose of this paper is to formally introduce this currently practiced diagrammatic notation to the broader community, provide a guide on its use, and discuss its state as a diagrammatic language. As such, rather than this paper being a suggestion of new additions to our diagrammatic notation, it is instead a presentation of its current state of development. We discuss how the notation provides visualization tools for various epistemic entities and relations posited in theoretical scientonomy. This diagrammatic notation is best viewed as an ongoing project, as we modify and refine it in response to changes in theoretical scientonomy, developments of diagramming techniques in other notations, and applications to a growing range of episodes.

The paper is structured as follows. It begins with an overview of the scientonomic ontology of epistemic entities and relations. It then provides basic visualization techniques for each of these entities and relations. With these basic techniques at hand, it then presents a variety of diagram types that can visualize belief systems synchronically and diachronically. After presenting the current state of the diagrammatic notation, the paper outlines some directions for further research. 


\section{Epistemic Entities and Relations}

For a set of diagrams to qualify as a system, it must be based on a solid theoretical foundation stating what types of entities, relations, actions, processes, etc. populate the respective domain and, therefore, what types of entities, relations, actions, processes etc. need visuographic depiction. Without such a shared theoretical foundation, we would have nothing more than a loosely connected set of visualization techniques. Thus, it has to be based on the ontology of the domain in question. Specifically, this means that our notation should reflect the ontology of epistemic elements, stances, agents, and relations between them currently accepted in theoretical scientonomy. For instance, if the ontology of theoretical scientonomy says that a theory is an answer to a question, then there should exist a diagrammatic way of depicting that relation. Similarly, if the ontology of theoretical scientonomy tells us that a theory can be accepted, used, and/or pursued, our notation should provide visual means for depicting a theory's acceptance, use, and/or pursuit. It is also clear that, as the theoretical ontology of scientonomy evolves, so should do any diagrammatic notation based on it. If, for instance, a new type of entity or relation is accepted into the scientonomic ontology, the diagrammatic notation should be amended accordingly to reflect this addition. This situation is not unique to visualizing belief systems. In fact, any systematic notation based on a solid theoretical foundation faces the challenge of keeping itself up to date with the developments in its theoretical basis; consider, for instance, the developments of diagrammatic notations in physics (Kaiser, 2005) or The Systems Biology Graphical Notation (Le Novère et al., 2009).

This brings us to our first task: identify every entity and relation currently accepted as part of the scientonomic ontology. Central to the scientonomic ontology is the idea of an epistemic agent taking an epistemic stance towards an epistemic element (Barseghyan, 2018; Patton, 2019). As such, any historical record concerning the content of a belief system is a statement of a certain agent - individual or communal - taking a certain stance (e.g., accepting, using, pursuing, employing) towards a certain epistemic element (e.g., a theory, method, question). Naturally, there are several types of agents, elements, and stances. In addition, a variety of relations can obtain between epistemic elements, between epistemic elements and epistemic agents, as well as among different epistemic agents. We shall start, therefore, with a quick outline of these entities and relations. The outcome of this exercise is a comprehensive laundry list of entities and relations that need to have their corresponding diagrammatic representations.

According to the current scientonomic ontology, the two fundamental types of epistemic elements that populate belief systems are questions and theories, where questions are understood as topics of inquiry and theories are understood as sets of propositions (Rawleigh, 2018; Barseghyan, 2018):
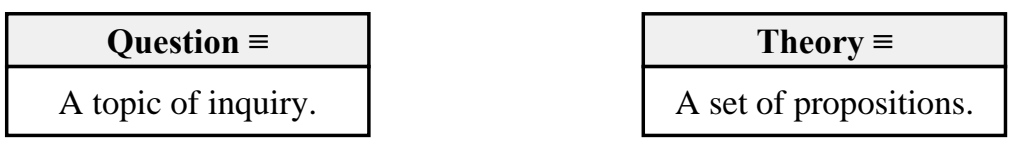

The latter category includes both individual propositions such as "The ocean surface temperature measured at $15.1^{\circ}$ north latitude and $30.6^{\circ}$ west longitude in the Atlantic Ocean at 9:00 AM Eastern Standard Time on June 12,2004 was $28.2^{\circ}$ Celsius" and general propositions such as "The burning of fossil fuels by human beings leads to global warming". Thus, the class of theory includes not only elaborate theories from climatology, physics, biology, sociology, or economics, but also individual data points obtained as a result of observations and experiments. Theories can be of three types - definitions, descriptive theories, and normative theories (Barseghyan, 2018): 


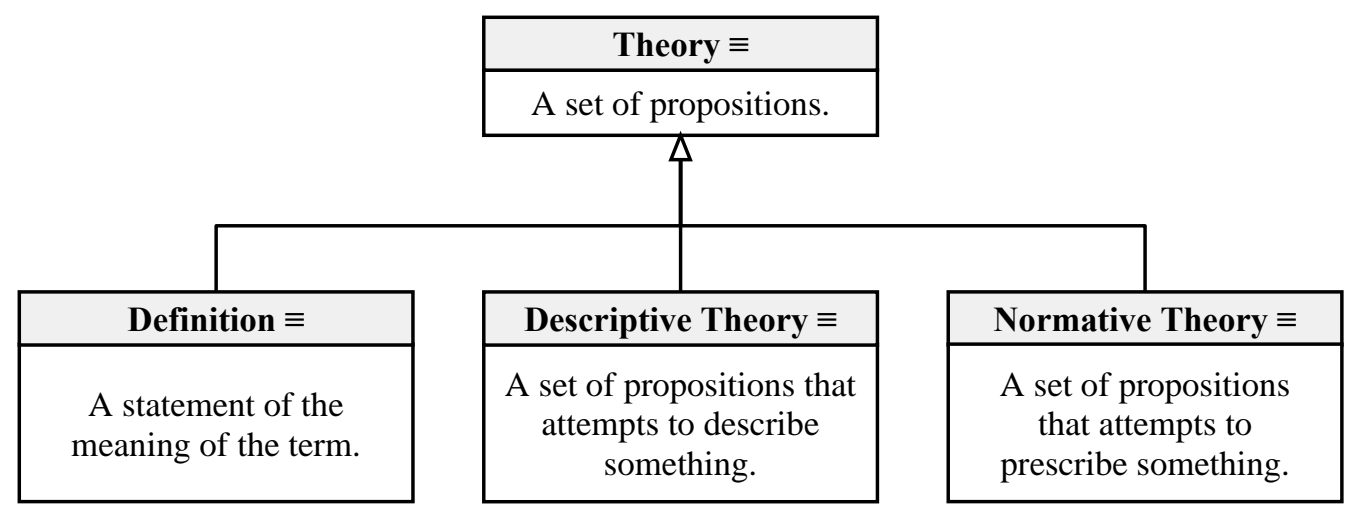

These different types of theories are answers to different types of questions:

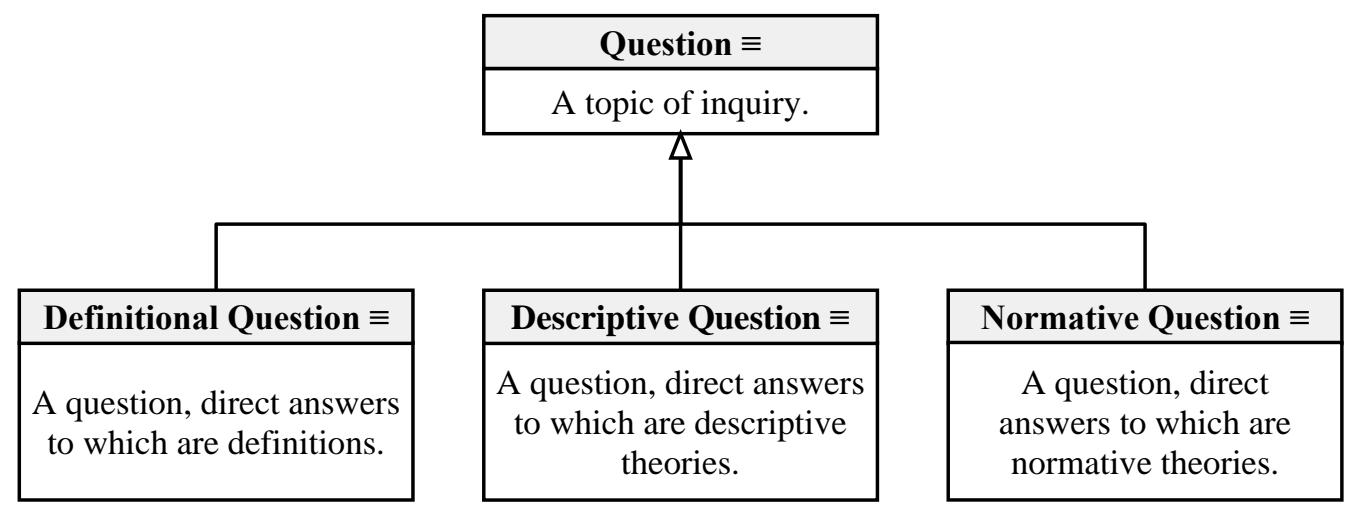

It is also accepted, that methods of theory evaluation are a special subtype of normative theories, along with ethical norms, aesthetic norms, etc. (Barseghyan, 2018):

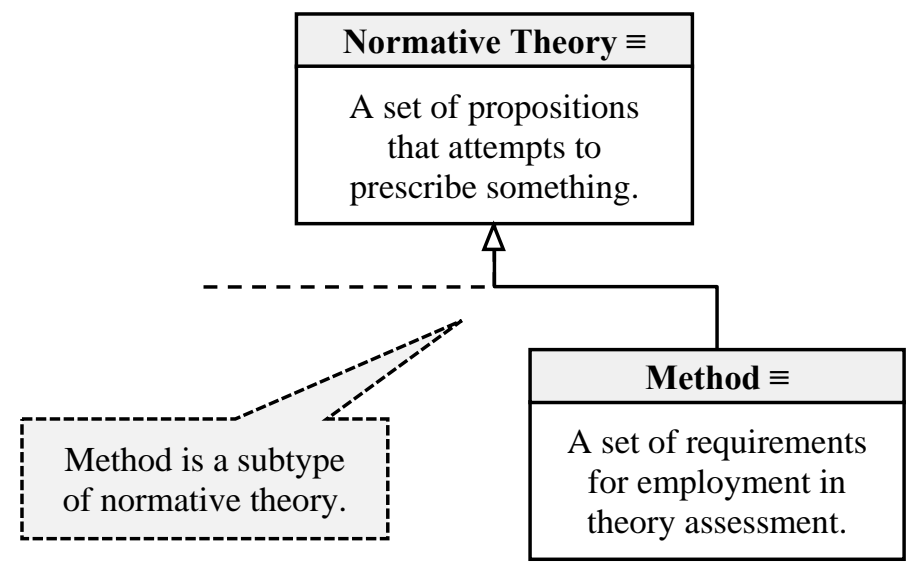

Epistemic elements stand in a variety of relations with one another that require diagrammatic depiction. We will first consider some relations that can exist between theories. These include:

- implication: a theory can logically follow from some other theory or theories (the opposite is nonimplication);

- biconditional: two theories can be equivalent from the perspective of their epistemic content (the opposite is exclusive disjunction);

- consistency: two theories can be logically consistent with one another (the opposite is inconsistency)

- compatibility: two theories can be epistemically compatible, i.e., they can coexist in the same mosaic (the opposite is incompatibility; see Barseghyan, 2015, pp. 152-164; Fraser \& Sarwar, 2018). 
More broadly, sets of theories are typically enlisted to form logical arguments. For example, theories may act as premises of a deductive argument with another theory as its conclusion. Theories may thus function as reasons for the acceptance of other theories (Palider, 2019). Understanding the complex relations among theories that exist when they are called upon to function as parts of logical arguments for other theories can be greatly facilitated by their diagrammatic depiction.

Additionally, there are certain relations that may hold between specific subtypes of theory. Definitions, for example, normally comprise a system, or a taxonomy, by standing in two types of relations with one another:

- generalization/specification: a concept can be a subtype of a more general concept, or a supertype of a more specific concept (e.g., method is a subtype of theory, while theory is a supertype of method);

- $\quad$ use: a concept can be used to define another concept (e.g., the concepts of acceptance and employment are used to define the concept of mosaic).

The latter relation can also hold between a definition and a theory of any other type, as definitions are used in the formulations of all theories. For instance, the current formulation of the second law of scientific change uses, among others, the definition of method, theory acceptance, and assessment outcome.

A relation specific to normative theories such as methods is that of implementation: a method can be a more

The scientonomic notion of method should not be confused with a common usage of the term in scientific practice. The methods section of a scientific paper, for example, describes procedures and tools involved in the conduct of a scientific experiment; these procedures and tools being its "methods". When we speak of the concrete implementation of a method, we are, rather, speaking of the specific criteria that a theory should satisfy to be accepted by an agent. concrete implementation of the requirements of a more abstract method. For instance, the requirement to test drugs in double-blind trials is one concrete implementation of a more abstract requirement to avoid experimenter bias in the testing of drugs (Barseghyan, 2015, pp. 7-8, 151-152). This implementation relation will also be a target for visualization.

In addition, there are two fundamental relations that hold between theories and questions (Rawleigh, 2018; Barseghyan, 2018; Barseghyan \& Levesley, 2021):

- a theory is an attempt to answer a question, while each question can have many attempted answers (e.g., the propositions "God exists" and "God does not exist" are both attempts to answer the question "Does God exist?");

- a question can presuppose one or many theories (e.g., the question "What is the distance between the center of the universe and the sphere of the stars?" presupposes such theories as "The universe has a center" and "The sphere of the stars exists").

As both of these relations are fundamental in an analysis of a belief system, we must have a way of diagrammatically depicting them.

According to the scientonomic ontology, epistemic agents can take epistemic stances towards epistemic elements. The typology of these stances varies for different epistemic elements. Thus, according to the scientonomic ontology of epistemic stances, questions can be accepted (the opposite is unaccepted), while theories can be accepted, used, and pursued (the opposites are unaccepted, unused, and neglected, respectively). In addition, normative theories, such as ethical norms, aesthetic norms, or methods, can also be employed.

An epistemic stance is, in essence, a temporal relationship between an agent and an element. Thus, these relations are normally captured in historical records that indicate which agent took which stance towards which element. For instance, the historical record "General relativity is currently accepted by the physics community" involves the agent (the physics community), the element (general relativity) and the stance (acceptance). Or consider the record "The Aristotelian method of intuition schooled by experience was employed by natural philosophers since circa 1300 till circa 1700". This record too indicates the agent (natural philosophers), the element (the method of intuition), and the stance taken towards that element (employment). In addition, historical records state the time or the period when a certain stance was taken by an agent towards an element (e.g., "currently", "from circa 1300 till circa 1700"). Epistemic stances can be taken explicitly or implicitly (Barseghyan, 2018). While in many cases an agent's stance towards a certain element is clearly stated and can be reconstructed 
from the written record, at other times the agent's stance can only be deduced indirectly. Ideally, we need to have a diagrammatic way of expressing the difference between these two situations.

It is important to keep in mind that the available historical data is often imprecise. Consider for instance a historical record "Newton's theory became accepted in England circa 1700" or "French chemists accepted the theory of caloric sometime between 1785 and 1789". In addition, sometimes historical records reveal that at a certain date an agent had a certain stance towards some theory, method, or question, but they are silent on when exactly that stance was first taken by that agent or when the agent ceased to take that stance. In other words, sometimes we are lacking historical data on the exact period when the stance was taken. Our notation should be able to clearly convey such uncertainties.

Central to the scientonomic ontology is the notion of an epistemic agent. An epistemic agent is an agent capable of taking epistemic stances towards epistemic elements based on a semantic understanding of the element and its alternatives. The current scientonomic ontology accepts the existence of two fundamental types of epistemic agents - individual agents and communal agents (Patton, 2019). While the former category includes individuals such as Aristotle, Aquinas, Descartes, or Einstein, the latter category includes communal entities that are equally capable of taking epistemic stances, such as the Intergovernmental Panel on Climate Change, the contemporary physics community, or the community of $18^{\text {th }}$ century French chemists. This means that both communal and individual agents can have mosaics or, more generally, can take epistemic stances towards epistemic elements.

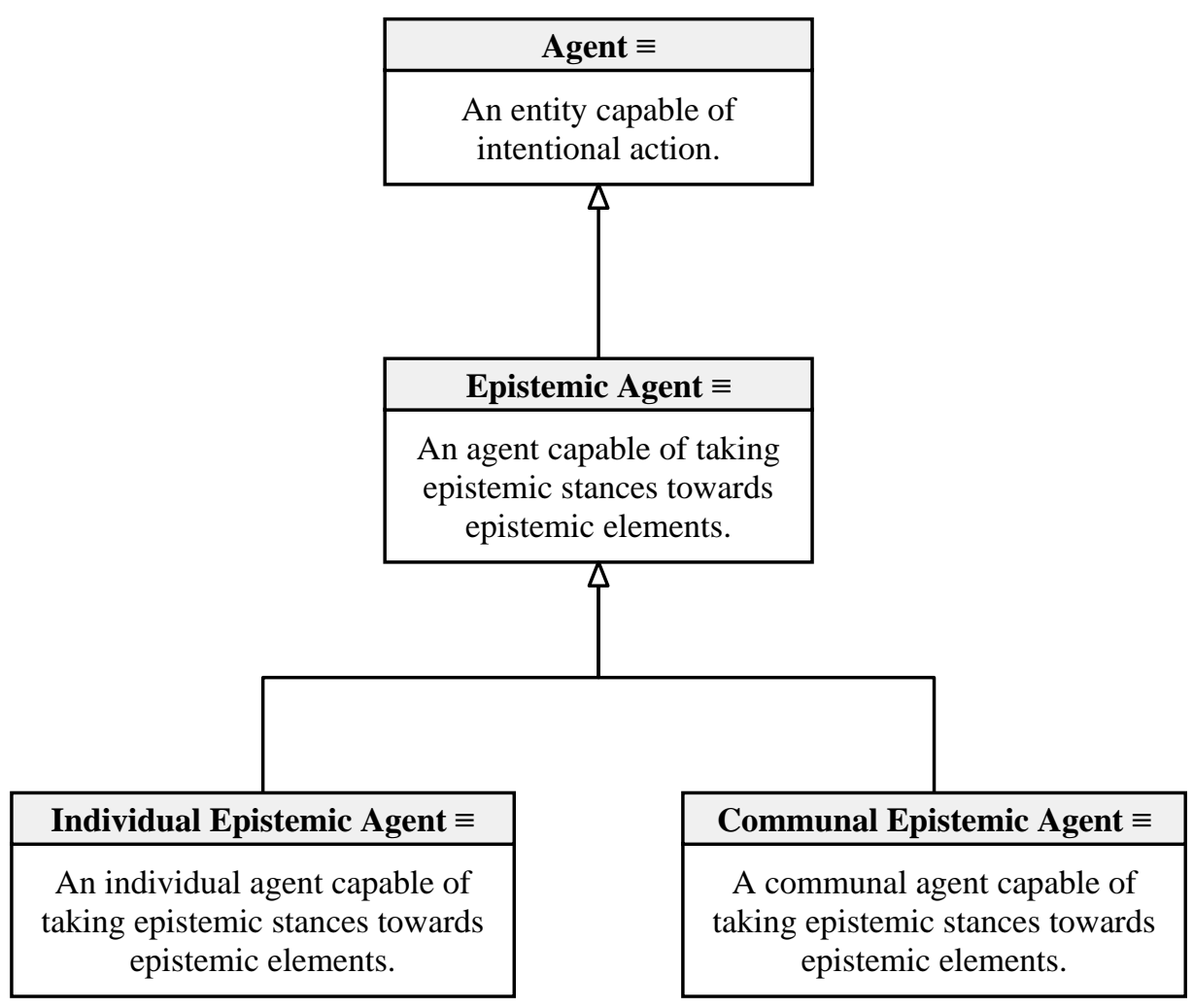

Epistemic communities may involve a division of epistemic labor between agents with differing areas of special expertise. Communal agents can thus consist of other communal and individual agents, i.e., a community can be a subcommunity of another community, just as an individual can be a member of a community. Depicting these relations might be helpful for illustrating the organizational hierarchy of communities, subcommunities, and individuals. 
Another relation that can obtain between agents is that of authority delegation (Overgaard \& Loiselle, 2016; Patton, 2019):

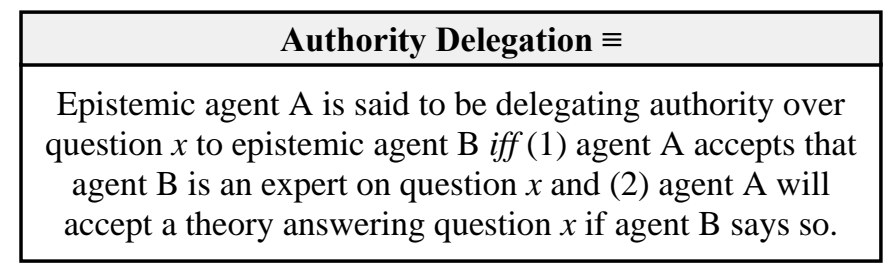

When an agent delegates authority over a certain topic to several expert-agents, this delegation is often hierarchical in nature, i.e., these experts are often delegated different degrees of authority over the topic (Loiselle, 2017). When analyzing an epistemic situation, it is often important to extract the intricate network of authority delegation relations between the respective agents. Thus, it is important to provide a means to depict these relations diagrammatically.

Epistemic elements can be grouped into collections in a variety of ways, depending on the stances taken towards them the by an agent in question. One such grouping is an agent's mosaic, which is defined as a set of all epistemic elements accepted and/or employed by an epistemic agent (Barseghyan, 2018). Thus, an agent's mosaic includes all of their accepted questions, accepted theories, and employed norms. For example, the contemporary physics mosaic will include not only the descriptive propositions of theories such as general relativity, but also all the questions to which these propositions attempt to answer, as well as all the norms employed by this community, including but not limited to their methods of theory evaluation. Most of the relations that obtain between theories can also obtain between mosaics. These include the relations of equivalence (biconditional), consistency, and compatibility. Besides these relationships, it would be helpful to visualize the overlaps between two or more mosaics.

Mosaics of communal agents can split and merge. For instance, the schism between the Church of England and the Roman Catholic Church in 1534 can be interpreted as a mosaic split, where one theological mosaic resulted in two theological mosaics. Similarly, the acceptance of Newton's natural philosophy in France circa 1740 can be said to have led to the merging of the French and British natural philosophy mosaics. By visualizing such splits and merges, we can portray an important aspect of the process of intellectual change.

Note that while the current scientonomic ontology doesn't explicitly define grouping of elements other than mosaics, in principle one can conceive of a number of alternative ways to group epistemic elements, including but not limited to the set of all the theories pursued by an agent, the set of all the theories used by the agent, the set of all the norms employed by the agent and so on. It would be advisable to have a standardized way of depicting such groupings.

Finally, our diagrammatic notation will also require techniques for visualizing more generic entities and relations. For example, historians often find it important to trace epistemic influences between agents, such as "Beeckman influenced Descartes" or "Descartes influenced Newton". Therefore, it might be useful to have a diagrammatic way of depicting such influences. Historians, also often speak, metaphorically, of belief system $A$ having influenced belief system $B$. While this is merely shorthand for a complex tangle of causal influences exerted by agents (sometimes via tools such as written texts) upon one another, it would also be useful to visualize such influences. Also, many visualizations will most likely require visual elements of non-epistemic nature, such as callouts, highlighting, grouping, or captions. Whenever necessary, such visualization techniques will be introduced. Yet, they will be kept to a minimum to avoid both clutter and any considerable discrepancy with the scientonomic ontology. 


\section{Visualizing Epistemic Entities and Relations}

With the above laundry list of epistemic entities and relations at hand, we can now proceed to their visualization. The structure of this section will - for the most part - reflect the structure of the preceding section to ensure that visualization tools are provided for each epistemic entity and relation.

When devising visualization techniques, it is important to keep in mind that the resulting diagrams must be accessible to as many people with visual disabilities as possible. Such accessibility considerations impose a number of limitations on diagrammatic techniques that one can use to depict belief systems. Specifically, these considerations suggest that none of the following should be used to convey any semantic content:

- line width: the thickness of a line should make no semantic difference;

- color: no semantic content is to be covered by differences in color, including low-contrast shades;

- fill pattern: there should be no semantic difference between solid-fill and patterned-fill elements;

- font: text font, size, and style (bold, italic, underline, etc.) should be irrelevant.

Thus, our diagrams should not convey any semantic content by means of line widths, colors, shades, fonts, or fill patterns. Instead, they will contain high-contrast accessible visualizations. Note, however, that it is acceptable to use colors, fonts, and patterns for aesthetic and highlighting reasons provided that they do not perform any semantic function (see section Highlighting and Callouts below).

\section{Epistemic Elements}

In scientonomy, all epistemic elements are usually depicted in rectangles (with or without a title). This practice is borrowed from Universal Modeling Language (UML), a diagrammatic language devised by software engineers to represent program elements, in which both objects and classes of objects are represented by rectangles (Seidl et al., 2012, p. 53).

Title and Body

If the title of the element in question needs to be shown, the rectangle can come with a title box. Here is a typical depiction of a theory with a title (Barseghyan, 2015, p. 223):

\begin{tabular}{|c|}
\hline Dynamic Substantive Methods \\
\hline $\begin{array}{c}\text { All substantive methods are } \\
\text { necessarily dynamic. }\end{array}$ \\
\hline
\end{tabular}

Note that, unlike formulations in the body, titles do not end with a period.

For aesthetic reasons, the title in the box should be capitalized and should - whenever possible - not exceed one line. In other words, the height of the title should not be resized. Thus, the following is not advisable:

\begin{tabular}{|c|}
\hline $\begin{array}{c}\text { Dynamic Substantive } \\
\text { Methods }\end{array}$ \\
\hline $\begin{array}{c}\text { All substantive methods } \\
\text { are necessarily dynamic. }\end{array}$ \\
\hline
\end{tabular}

Instead, the rectangle of the element should be resized horizontally to ensure that the whole title fits in one line.

At times, it might be necessary to omit the title and simply state the formulation of the element. Consider the following depiction:

All substantive methods are necessarily dynamic. 
Alternatively, it might be necessary to omit the exact formulation in a diagram and include only its title. In such a case, the title should be capitalized but not bolded. One consideration against bolding the titles in this usage is that this may result in excessive amount of bold text on diagrams involving a large number of such elements (e.g., taxonomies). Thus, the usage we suggest is illustrated below:

Dynamic Substantive

Methods Theorem

In addition to its aesthetic virtues, this depiction is notably more compact than the bolded version. It is thus especially handy in larger diagrams that show interconnections between many different epistemic elements where space is of the essence. In this depiction, it is acceptable and even advisable for the title to run multiple lines in order to maintain an aesthetically pleasant ratio of the rectangles' width and height.

\section{Implicit \& Explicit}

It will sometimes be necessary to indicate that certain epistemic elements were stated explicitly while others were

In some early scientonomic works, all theories were depicted with solid outline rectangles and all methods with dashed outline rectangles (Barseghyan, 2015, pp. 56, 135). This convention has since been superseded.

1930's, the following assumption was implicitly accepted by the physics community as part of classical physics only tacitly assumed. In such diagrams, implicit elements are to be depicted in dashed rectangles, and explicit elements with solid lines. For example, before Einstein formulated his criticism of quantum mechanics in the

(Fine, 2017; Barseghyan, 2018):

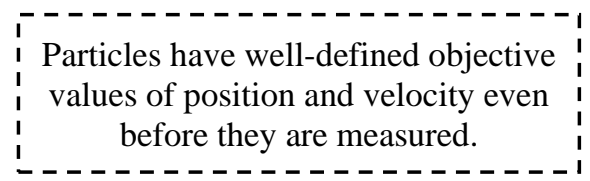

In addition to descriptive theories, it is also sometimes the case that normative theories, and in particular employed norms, are often left implicit. Among other things, depictions which distinguish explicit from implicit theories may be useful for showing how certain tacit assumptions became explicit over time. In those cases, when historians are unclear as to whether a stance towards a certain element was taken implicitly or explicitly, we recommend using solid rectangles. The rationale behind this choice is that normally it takes a considerable effort on the part of a historian to show that a certain element was accepted implicitly. If a historian merely says that a certain agent accepted a certain theory, then we can naturally assume that it is explicit acceptance that the historian has in mind.

Importantly, the explicitness/implicitness is relative not only to the agent in question but also to the body of textual evidence on which a particular diagram is based. It might so happen that in reality the agent explicitly stated a certain theory, but the given body of text does not make this clear. When presenting the agent's belief system in a diagrammatic form, it is important to stay true to what is found in the respective body of text. Therefore, one should not make any assumptions about the explicitness/implicitness of a certain theory in the agent's belief system that go beyond the texts under scrutiny. While it is possible that the respective agents did state these premises explicitly, one should refrain from making such assumptions and should try to stay true to the texts at hand. Thus, our recommendation is to portray these assumptions as tacit, i.e., in dashed rectangles.

Indicating tacit premises becomes especially important when reconstructing specific arguments of some agent. Often, the source material does not provide spell out all the premises necessary to reconstruct a valid argument. In such cases, implicit premises may be inferred, if it would make sense for the agent in question to accept such premises. To some degree, this is of course a matter of subjective interpretation by the writer of the source materials and the creator of the diagram. Diagrammatically noting that certain premises are implicit while others are explicit can make it easier to critique an interpretation. Erroneous interpretations can be more readily identified 
and corrected. For example, consider the implicit premises assumed to be accepted by the Aryan physics community during the reign of the Nazi regime in Germany (Rapson \& Shaw, forthcoming):

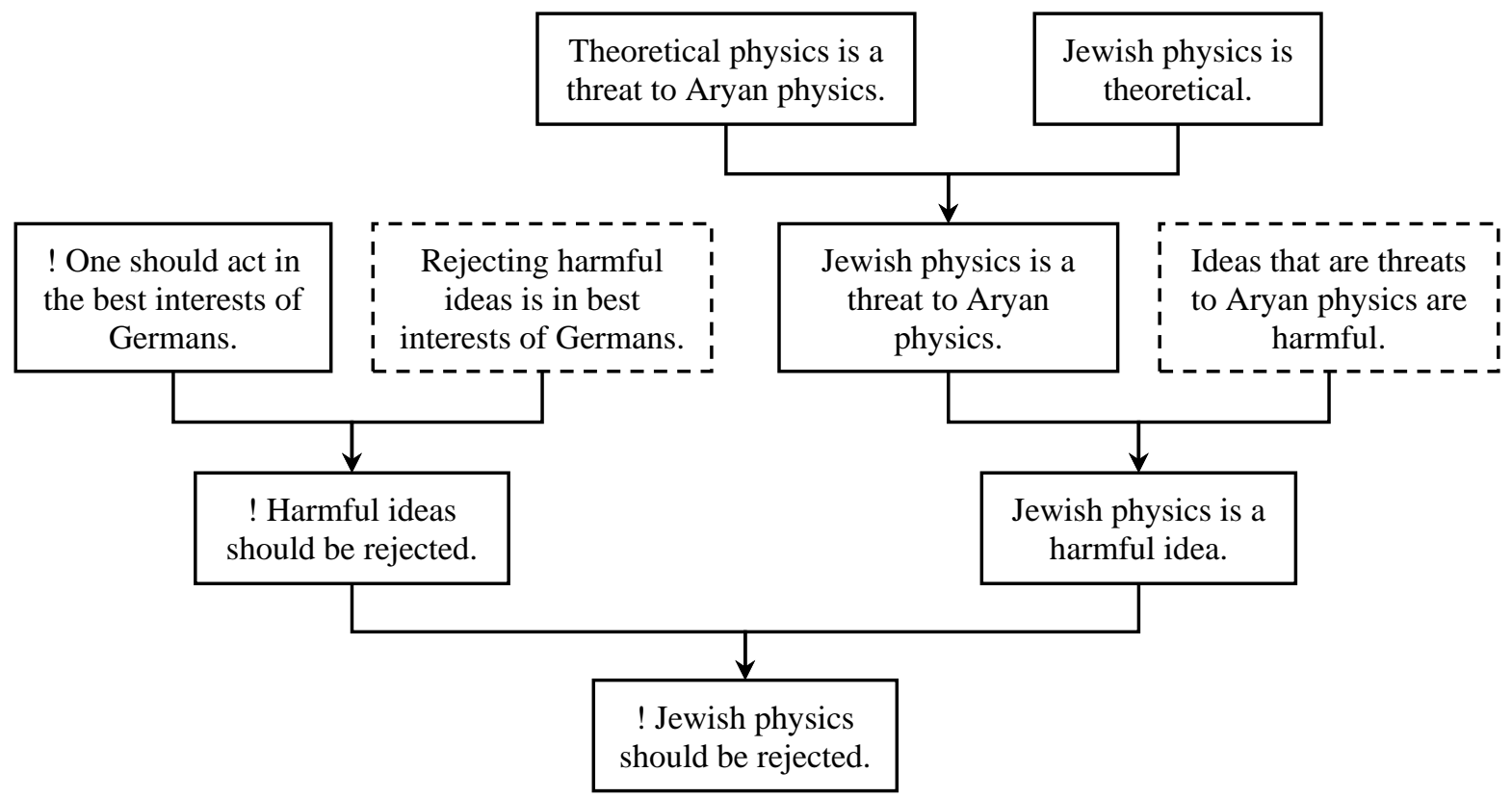

These implicit premises are needed to ensure the validity of the argument. Among other things, their explicit formulation raises important questions of their justification by the respective agent and invites future historical research.

One may wonder if there might be cases when it would be allowed to ignore the distinction between explicit and implicit and portray all elements in solid rectangles. We believe that it would always be useful to indicate which premises come directly from the source, and which are assumed by the writer. The requirement of consistency dictates that we should stick to a standard usage of solid/dashed rectangles to avoid unnecessary confusion.

Theories

According to the current scientonomic ontology, theories come in three subtypes: definitions, descriptive theories, and normative theories. Scientonomic diagrams have been explicitly specifying definitions by means of the triple bar symbol ( $\equiv)$, which is often used in mathematical equations to indicate that the symbol on the left-hand side of the equation is being defined (Lambek \& Scott, 1986, p. ix.). Here are some typical scientonomic definitions (Barseghyan, 2018, p. 15):

\begin{tabular}{|c|}
\hline Theory $\equiv$ \\
\hline $\begin{array}{r}\text { A set of propositions that attempt to } \\
\text { describe something. }\end{array}$ \\
\hline
\end{tabular}

\begin{tabular}{|c|}
\hline Method $\equiv$ \\
\hline $\begin{array}{c}\text { A set of requirements for } \\
\text { employment in theory assessment. }\end{array}$ \\
\hline
\end{tabular}

We suggest continuing this usage and include the triple bar symbol in the titles of all definitions. The only exception from this rule is those diagrams which include only concepts depicted in the title-only mode without their respective definitions. On such diagrams (see section A Taxonomy of Diagrams below), the triple bar symbol can be omitted to avoid unnecessary clutter.

Now, the question is whether we need to indicate the other subtypes of theories: do descriptive and normative theories also need a visual indication that they belong to their respective classes? We suggest using an exclamation mark (!) to indicate normative theories. This would be in tune with the traditional use of this symbol in deontic 
logic, in which it expresses "the imperative factor (or directive factor)" (Hilpinen \& McNamara, 2013, p. 23). Thus, a typical normative proposition would be depicted as follows:

\section{! Not Kill}

One ought not to kill

human beings.

The exclamation mark is also preserved in title-only depictions:

\section{! Not Kill}

When a normative proposition is depicted without a title, the exclamation mark should be added prior to the formulation (note, however, that in the full title-and-body depiction, the exclamation mark is present only in the title):

\section{! One ought not to kill} human beings.

This allows for normative propositions to be always easily detectable. We suggest sticking to this rule and only skipping the exclamation mark on those diagrams which depict exclusively normative theories. On such diagrams, it may be useful to skip the exclamation marks to avoid unnecessary clutter.

As a special type of normative theories, methods play a central role in scientonomy. Thus, one may wonder if it might be necessary to differentiate them from other normative theories with some special symbol. We believe that such a differentiation is not necessary for methods usually include word "method" in their title anyway. In addition, methods are rarely portrayed on diagrams which include other normative theories. The exclamation mark that distinguishes all normative propositions should therefore be sufficient. A typical method would be depicted as follows:

\section{! Blind Trial Method}

A hypothesis about a drug's efficacy is acceptable if the drug's effect has been confirmed in a blind trial.

While we believe that norms need to be visually indicated, it appears unnecessary to do the same for descriptive theories. For one, they are the vast majority of propositions in most belief systems, and we believe that indicating every single one of them with a special symbol would only add to the clutter. In addition, a visual indication of the other two types of theories - definitions and norms - should be sufficient to differentiate descriptive propositions.

\section{Infallibility}

In some cases, it might be necessary to show that a certain theory was thought to be infallible, i.e., it was accepted as a dogma, as something that is established beyond any reasonable doubt, etc. Consider for instance the typical medieval attitude towards "God exists". Not only was this theory accepted but it was also believed to be infallible. Scientonomically speaking, what we are dealing with here is the acceptance of two propositions - the first-order proposition "God exists" and the second-order proposition ""God exists" is an infallible truth". The following two diagrams are equivalent depictions of this epistemic situation: 


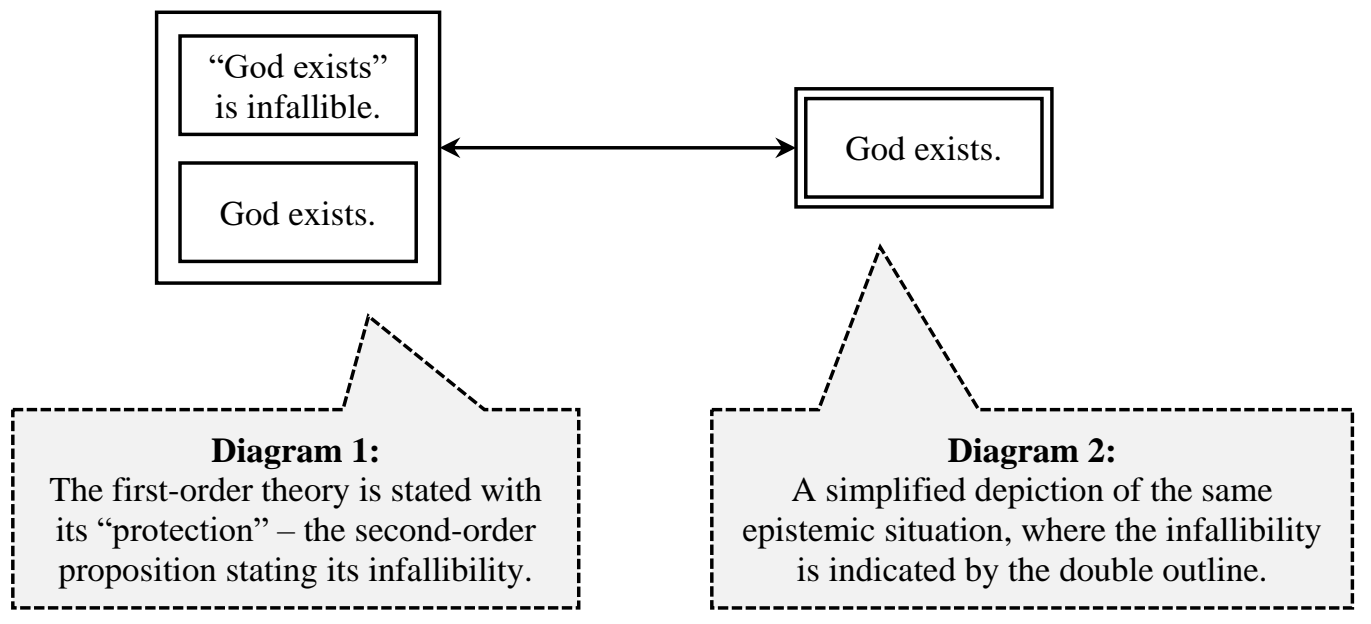

While both of these depictions are legitimate, the double-outline approach can greatly reduce the clutter by omitting the depiction of second-order theories stating "Proposition X is infallible".

It is also the case that in some epistemic situations a first-order proposition is stated explicitly while the second-order proposition of its infallibility is only implicitly assumed. To depict such situations, the internal outline should be solid, while the external outline should be dashed to indicate the implicitness of the secondorder proposition:

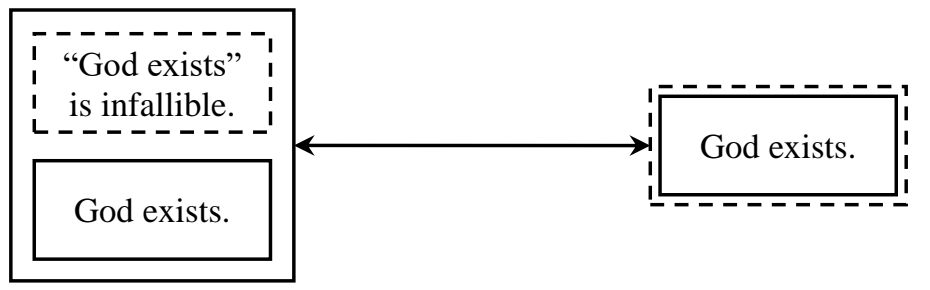

It is worth emphasizing that the right and left sides of this diagram are equivalent: any proposition $X$ in a doubleoutlined rectangle is to be understood as equivalent to a conjunction of two propositions - " $X$ " and " $X$ is infallible".

\section{Theory-Theory Relations}

While epistemic elements are depicted using rectangles, all epistemic relations are depicted by some kind of a connector between those rectangles with or without an arrowhead. For the sake of consistency, the connectors depicting epistemic relations are always elbowed, i.e., they follow the horizontal and/or vertical axes, and bend, when needed, at a right angle.

There are several logical relations between theories that have been visualized in scientonomy. These include the relations of implication, biconditional (equivalence), and compatibility together with their opposites. In The Laws of Scientific Change, a set of visualizations for basic logical relations is given (Barseghyan, 2015, p. 251). We have simplified this set of visualizations somewhat, to remove those never used, and propose the following set of connectors for basic theory-theory relations: 

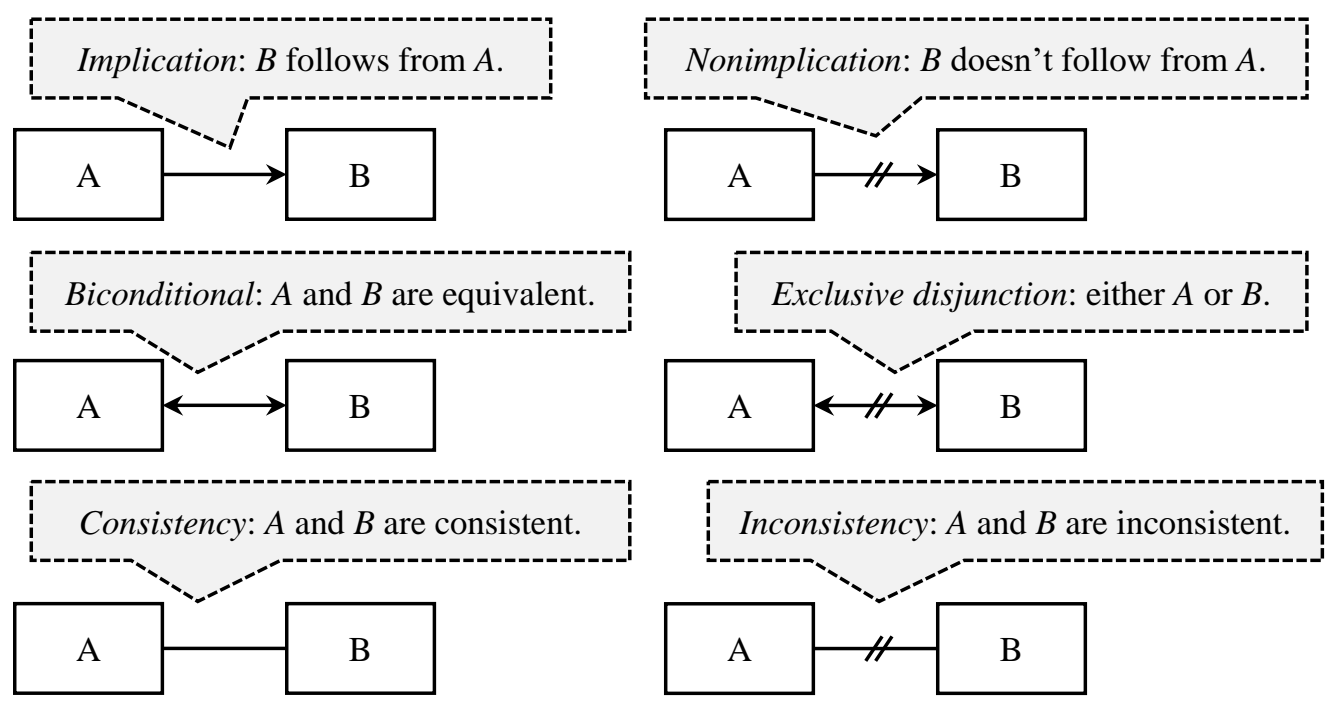

It is worth noting that the same solid arrow of implication can be used to denote the relation between more than two elements. If, according to a certain agent, theory $C$ follows logically from the conjunction of $A$ and $B$, it can be portrayed as follows:

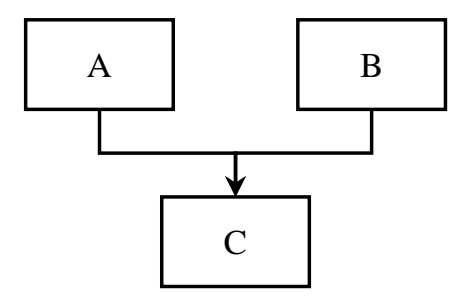

Note how, in this diagram, the two implication lines start from the two premises and then elbow to join and reach the conclusion box together. This depiction is not equivalent to the one when the two lines reach the conclusion box separately. In the latter case, it is understood that conclusion $C$ follows from premise $A$ and also follows independently - from premise $B$ :

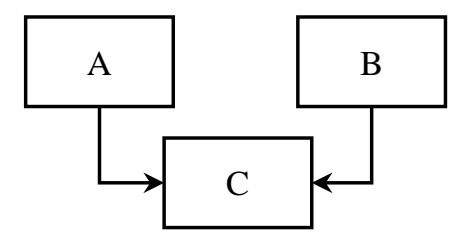

This type of diagram is useful for portraying a situation when a theory is believed to have several independent supporting reasons. Note that we do not currently distinguish between deductive and inductive implication.

When it is important to indicate that some of the premises of the argument are omitted from the diagram, an open-ended dashed connector can be used. In the following diagram, the dashed line indicates that there are other premises in play which have not been included in the diagram:

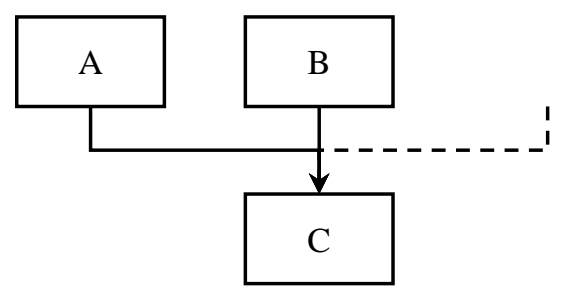


It is also noteworthy that, in the current scientonomic practice, the same connector is used to depict both consistency between two theories and compatibility between theories. However, we know that it is possible for an agent to consider a pair of theories as mutually inconsistent but compatible. One example of this is the current physics community's stance on general relativity and quantum mechanics. If we were to portray the current epistemic situation by means of the connectors that are currently in use, we would get the following picture:

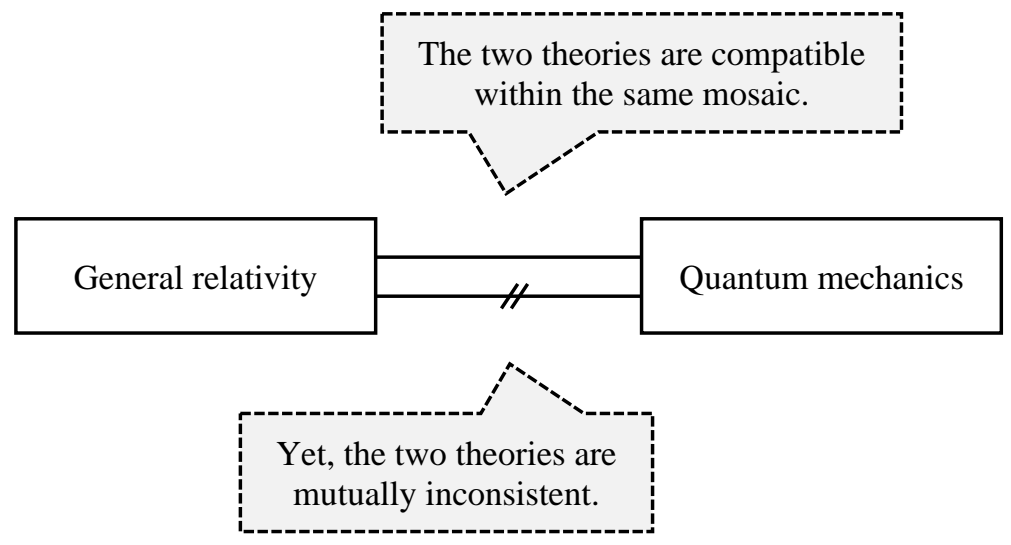

Clearly, such a depiction would be confusing.

Moving forward, we suggest using a dashed line for compatibility and a solid line for consistency. Correspondingly, the double-crossed dashed line will represent incompatibility, while the double-crossed solid line will be the connector for logical inconsistency.

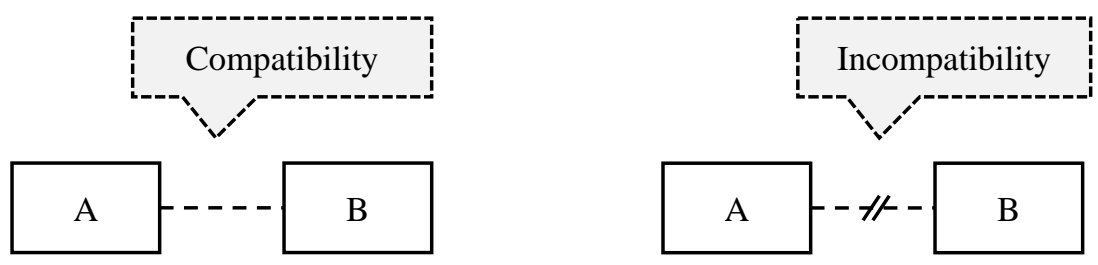

Thus, the above epistemic situation will be portrayed as follows:

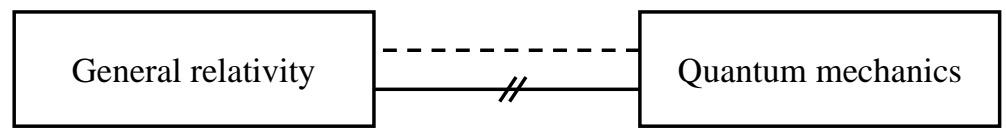

For the sake of consistency, we strongly suggest distinguishing between the connectors for compatibility/incompatibility and consistency/inconsistency at all times.

\section{Theory-Method Relations}

There are two types of relations between theories and methods currently accepted in theoretical scientonomy. First, by the third law, methods are deductive consequences of accepted theories (and other methods). This relation has been traditionally depicted in theory-method diagrams, like the one below (adapted from Fatigati, 2017, p. 26): 


\begin{tabular}{|c|c|}
\hline Theory & Method \\
\hline & $\begin{array}{l}\text { ! A proposition can be said to be accepted } \\
\text { in MASM if it is indicated as true in } \\
\text { authoritative texts taught in MASM. }\end{array}$ \\
\hline \multirow{4}{*}{$\begin{array}{l}\text { Licenses to teach [ 'ijāzāt] are reliable } \\
\text { indicators of which texts were } \\
\text { considered authoritative in MASM. }\end{array}$} & \\
\hline & $\downarrow$ \\
\hline & ! Teaching License Method \\
\hline & $\begin{array}{c}\text { A proposition can be said to be accepted } \\
\text { in MASM if the evidence of the licenses } \\
\left.\text { to teach [ }{ }^{\prime} i j \bar{a} z \bar{a} t\right] \text { indicates so. }\end{array}$ \\
\hline
\end{tabular}

In this diagram, a standard arrow is used to denote the deduction.

Second, a theory can be assessed by a method; as a result, it either satisfies the requirements of the method, fails to satisfy the requirements, or the assessment outcome is inconclusive (Patton, Overgaard, \& Barseghyan, 2017 , p. 31). Thus, sometimes it is necessary to show that a certain theory is in the mosaic because it satisfies the requirements of a certain method. It is important to realize that such a relationship is not one of logical implication. There is no way to deduce, say, general relativity from the HD method even when coupled with the respective observations (e.g., Eddington's 1919 observation of light bending). What can be deduced from the conjunction of a method and a respective observation is that the theory is acceptable. Here is an example:

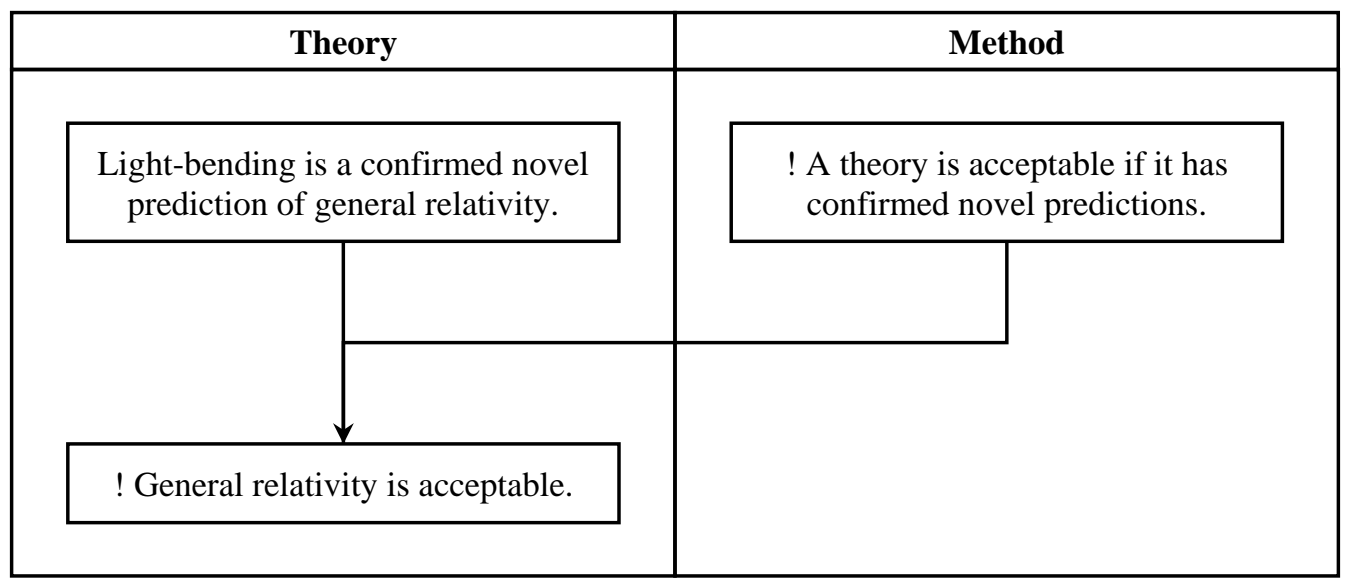

What is deduced here is a normative proposition of the acceptability of general relativity; what is not deducible is general relativity itself with all of its complex system of propositions. Yet, it is often necessary to show that a certain theory is in an agent's mosaic due to the fact that it satisfies the agent's method. We are dealing with a different relation here and the question is how exactly this relation is to be depicted.

Our suggestion is to depict this relation by using a dashed arrow with a corresponding label reading "satisfied". Here is an example:

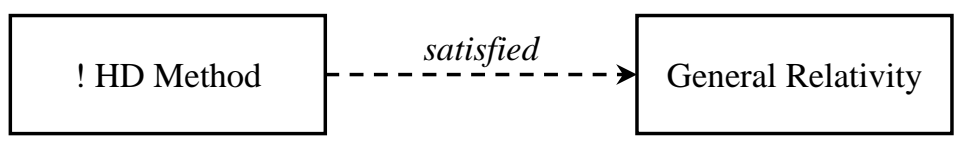

Note that the arrow goes from the method to the theory that was assessed by the method. If necessary, the results of respective observations/experiments can also be displayed: 


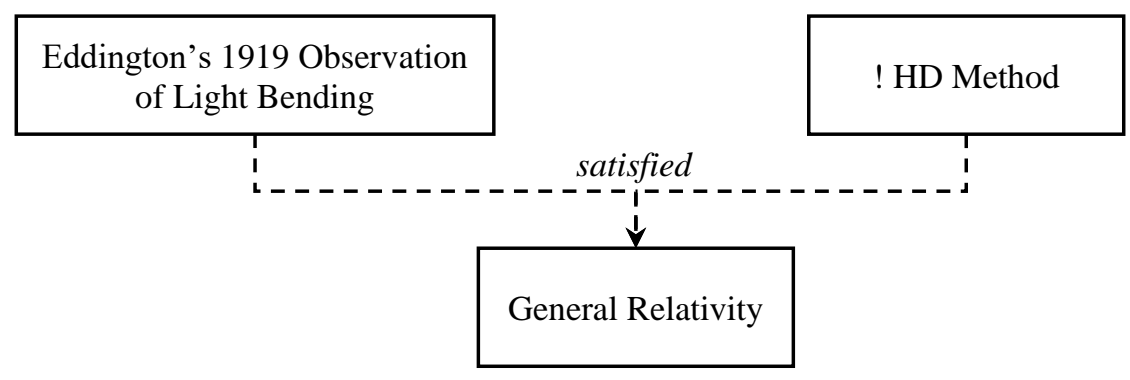

The relation of satisfaction may also be useful when simultaneously depicting changes in theories and methods on a timeline (for some examples, see section Diachronic Diagrams below).

\section{Method-Method Relations}

It is currently accepted in theoretical scientonomy that methods come in different degrees of concreteness/abstraction. Here is a diagram illustrating the point (adapted from Barseghyan, 2015, p. 8):

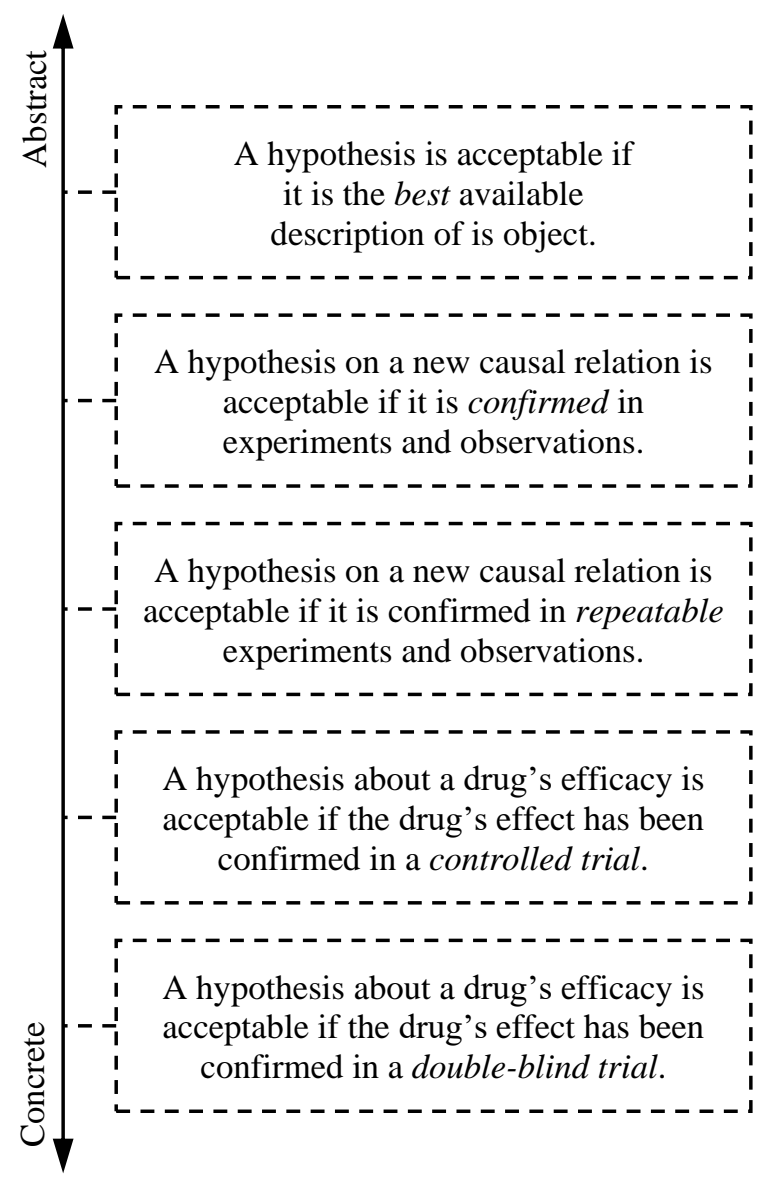

Note that, since all the propositions in the diagram are normative, the exclamation marks indicating normative propositions are omitted to reduce clutter.

It is also accepted that more concrete methods can be conceived as specific implementations of more abstract methods. This relation of implementation has been traditionally depicted by a dashed line with a solid white arrowhead, the standard UML symbol for implementation/realization. Here is a depiction of the blind trial method implementing an abstract requirement (adapted from Barseghyan, 2015, p. 138): 

! When assessing a drug's efficacy, the possible placebo effect must be taken into account.

\section{! Blind Trial method}

A hypothesis about a drug's efficacy is acceptable if the drug's effect has been confirmed in a blind trial.

\section{Definition-Definition Relations}

When depicting a taxonomy, it is often necessary to indicate that a certain concept is a subtype of (or a supertype of) a more general concept. To depict such relations of generalization/specification the standard UML arrow for generalization/inheritance is used. Here is an example (Barseghyan, 2018, p. 18):

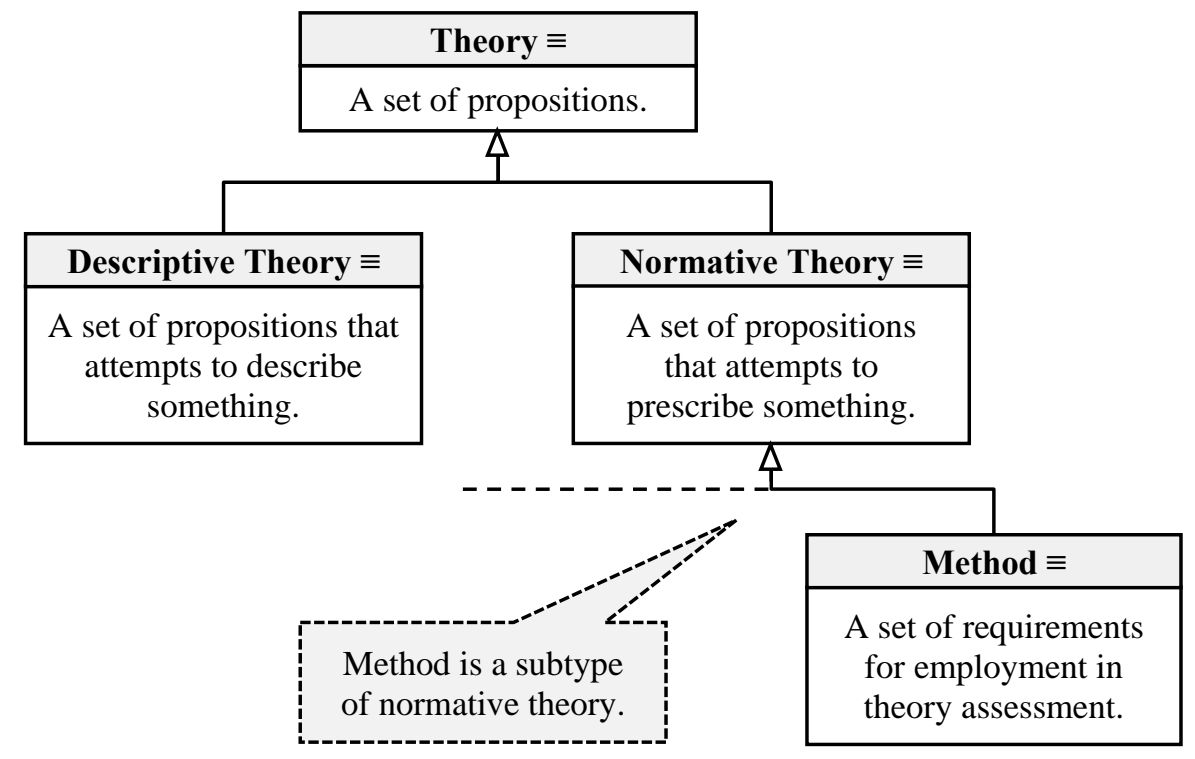

Also note the open-ended dotted line indicating that there are other subtypes of normative theories not depicted in the diagram. While always optional, such lines can highlight intentional omissions of some definitions from the diagram. In the above diagram, the dotted line highlights that other subtypes of normative theory, such as ethical and aesthetic norms, are intentionally omitted.

Every taxonomy will also have a plethora of cases when a certain concept uses another concept in its definition. For instance, the concept of authority delegation is defined by using a number of other scientonomic concepts, such as theory, community, topic/question, etc. Here is a depiction of these epistemic relations:

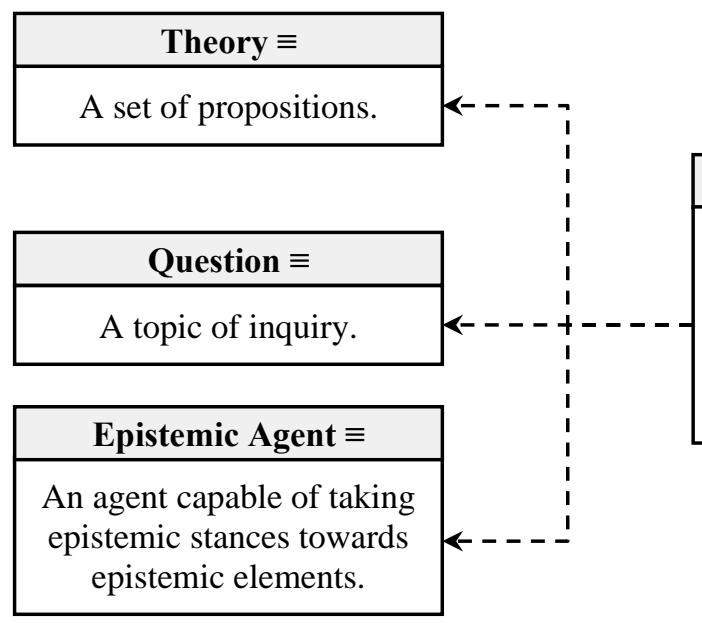

\section{Authority Delegation $\equiv$}

Epistemic agent $\mathrm{A}$ is said to be delegating authority over question $x$ to epistemic agent $\mathrm{B}$ iff (1) agent $\mathrm{A}$ accepts that agent $\mathrm{B}$ is an expert on question $x$ and (2) agent A will accept a theory answering question $x$ if agent B says so. 
If necessary, the subtype and use relations between different concepts can be depicted in the same diagram (provided that it does not introduce unnecessary clutter). Here is a diagram showing the subtypes of theory and also indicating that the concept of authority delegation uses the concept of theory in its definition:

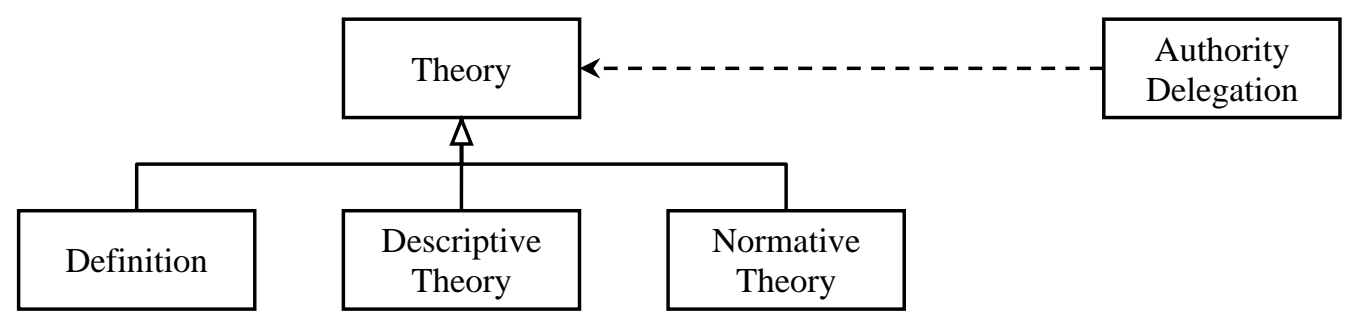

In addition to generalization/inheritance and use relations, two concepts can stand in the relation of aggregation. In the relation of aggregation, more objects are assembled or configured together to create a more complex object. For instance, the relationship between a team and its players is that of aggregation. Importantly, the individual components of the aggregate are capable of existing independently of the aggregate itself. Thus, players play for a team, but can move to other teams, or even have no teams. In UML, such relations are depicted by a connector with a hollow diamond (Seidl et al., 2012, p. 68):

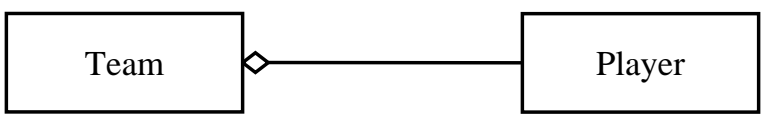

The relation in which the parts cannot exist without the whole is known in UML as composition. It is depicted in UML with a solid-diamond connector (Seidl et al., 2012, p. 68):

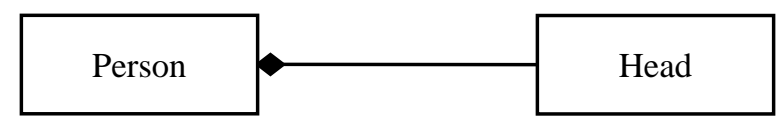

Compared to aggregation, composition is a stronger link between the whole and the part. In compositions, there is a strong lifecycle dependency between the whole and the part, where the latter cannot exist without the former.

Both aggregation and composition relations can be depicted with their respective multiplicities - one-to-one, one-to-many, many-to-many, etc. In UML, the multiplicities of relations are normally given as a minimum..maximum interval, where maximum and minimum can be any natural number or an asterisk $(*)$ when there is no restriction. When the values of maximum and minimum are the same, only one number is used to indicate the multiplicity. Also, keep in mind that $0 . *^{*}$ means the same as *, so $0 . *^{*}$ is never used, but $*$ is used instead (Seidl et al., 2012, p. 63). Here is an example:

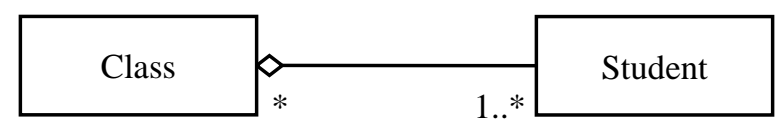

The diagram illustrates that any student can be enrolled in any number of courses, while each course can have one or many students.

The relations of both aggregation and composition are often found in agents' taxonomies. When a need arises to depict the relations of aggregation and composition, we suggest sticking to the standard UML graphical representation of these relations in our diagrams. Here is an example of a taxonomy that depicts such relations (adapted from Barseghyan, 2018, p. 34): 


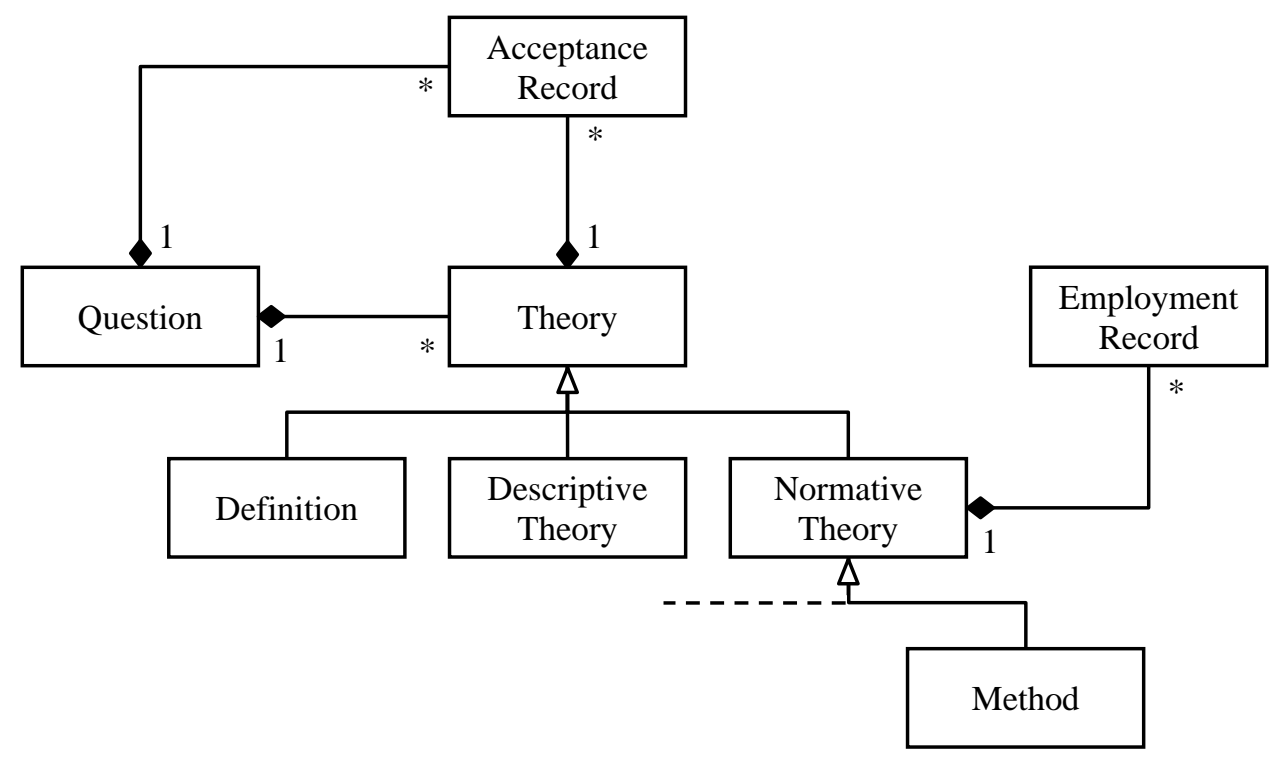

Among other things, the diagram illustrates that an employment record is always an employment record of some normative theory, while a normative theory can have any number of employment records. It also depicts similar relations between theory and question, between theory and acceptance record, as well as between question and acceptance record.

\section{Questions}

Like other elements, questions can be represented in three ways: with title and formulation, with title only, or with formulation only. To differentiate questions from other epistemic elements, we suggest using a question mark (?) at the end of the question's title, as follows:

\section{Kill ?}

Is it ever permissible to kill a human being?

The same applies to cases involving the title only:

Kill ?

For the cases where only the formulation of the question is depicted, in many languages no additional question mark will be necessary, since in these languages every formulation will naturally culminate with a question mark regardless:

\section{Is it ever permissible to} kill a human being?

In contrast, in those languages where interrogative sentences are not culminated with a question mark, it might be advisable to add an additional question mark - either after or before the formulation. Here is how a formulation of the same question would look like in Armenian:

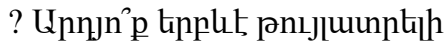

$$
\begin{aligned}
& \text { t5 ưnn uumuktil: }
\end{aligned}
$$


We have come to discover that such formulation-only depictions are most common on our diagrams; questions with tiles or title-only questions are relatively rare. However, in cases when such depictions are required, we recommend including a question mark in the title.

Now, the question is whether we need any special symbols to differentiate between different types of questions. Should we use something like (? $\equiv$ ) to indicate a definitional question, (?!) to indicate a normative question, etc.? Our suggestion is to refrain from using any additional symbols for differentiating between descriptive, normative, and definitional questions. The main consideration here is aesthetic, as adding any such symbols would contribute to cluttering diagrams. Thus, we suggest refraining from such usage and opting for the following depiction.

\section{Kill ?}

Is it ever permissible to kill a human being?

\section{Theory?}

What is theory? How should it be defined?

\section{Question-Theory Relations}

There are two types of relations between questions and theories. First, a theory is an attempt to answer a certain question. That relation has been depicted by using a standard UML symbol for composition (Barseghyan \& Levesley, 2021, p. 3):

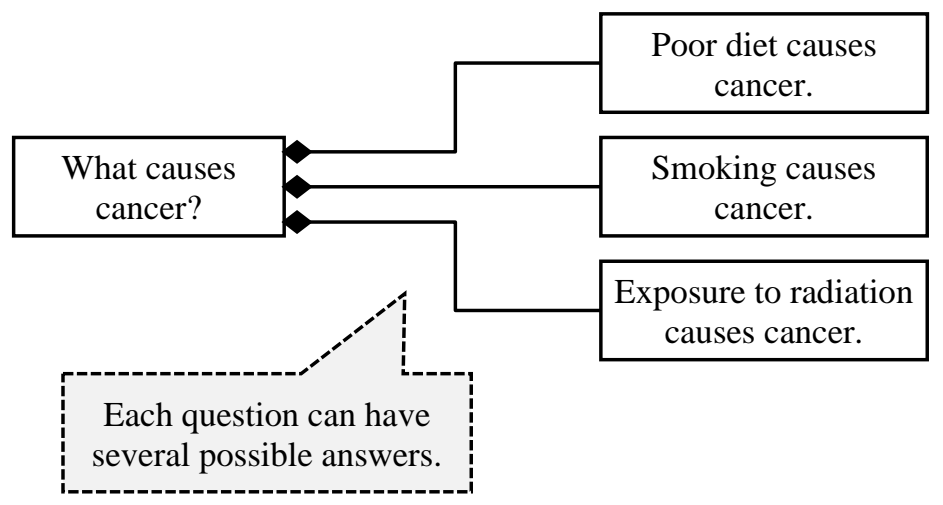

The fact that different answers to this question end at different diamonds indicates that these answers are not mutually exclusive: one can potentially accept both that poor diet causes cancer and that smoking causes cancer and that exposure to radiation causes cancer.

To indicate that an agent considered several answers to the same question as mutually exclusive, these answers should end at the same diamond, like in the example below:

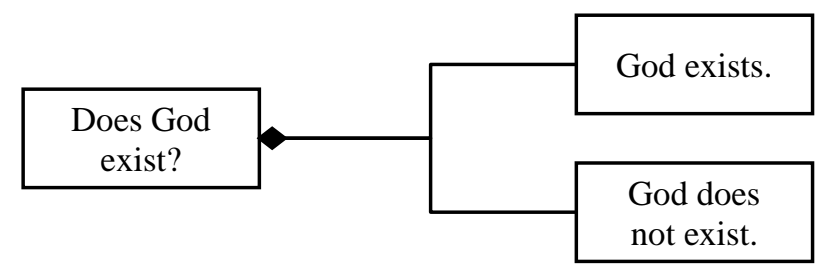

Second, a question can presuppose any number of other theories in order to be meaningful. Thus, in order for the question "what is the distance between the Earth hand the sphere of stars?" to even make sense, one has to accept the existence of the Earth and the existence of the sphere of stars. That relation has been depicted by means of a standard UML symbol for aggregation (Barseghyan \& Levesley, 2021, p. 4): 


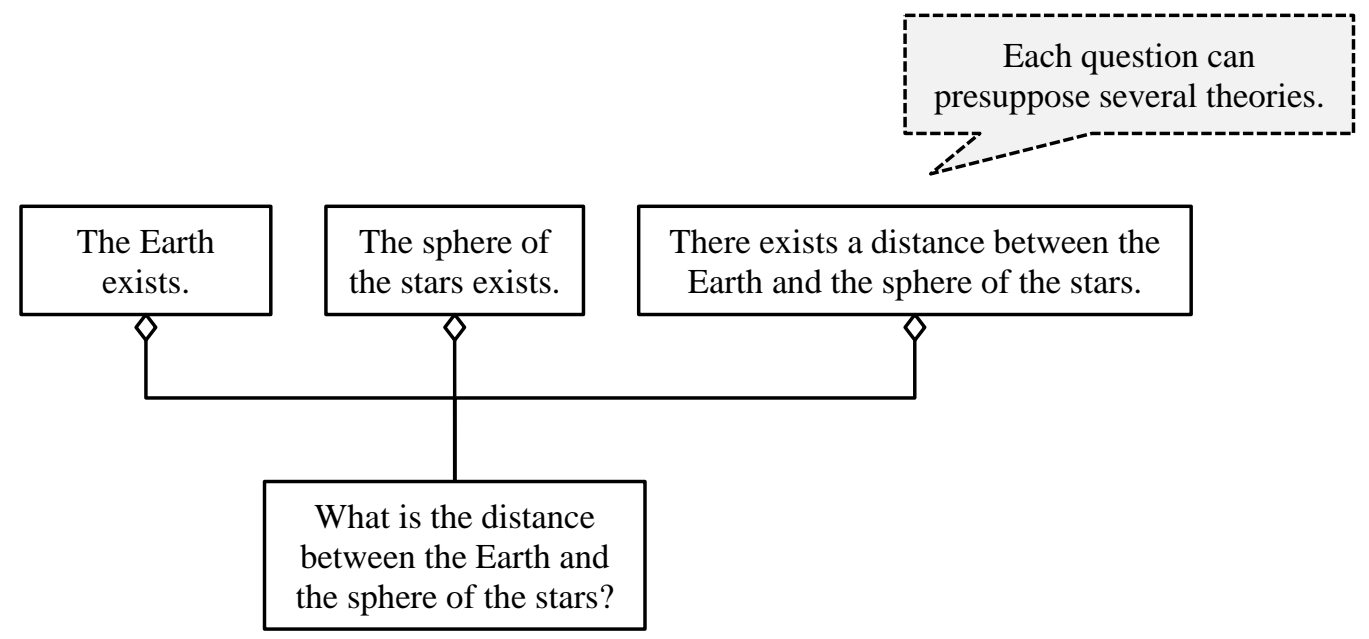

\section{Epistemic Stances}

As epistemic stances are temporal in nature, we have found that they are best depicted on timelines. We have experimented with other formats such as a traditional UML object diagram (see, for example, Barseghyan, 2018, p. 21), but have deemed this format to be cluttered and lacking in clarity, and so we don't recommend it. Here is an example of a simple timeline showing the acceptance and employment of the hypothetico-deductive (HD) method:

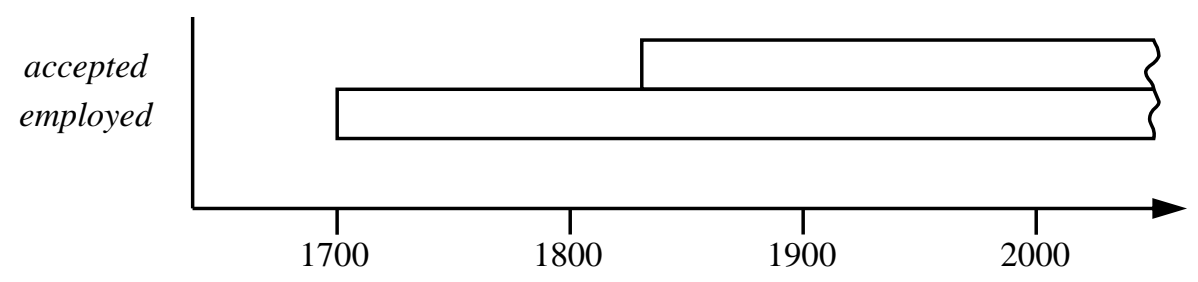

This depiction highlights the temporality of acceptance and employment, as this is typically the most salient aspect of historical epistemic stances. Note that wavy edges of interval bars indicate that the respective temporal intervals continue beyond the period captured on the timeline. In this diagram, the wavy edges indicate that the HD method is still accepted and employed. We suggest using wavy edges in all similar situations. In general, timelines allow for portraying both individual historical events and extended historical intervals. Thus, we can portray both the individual event of an agent changing their stance towards a certain element as well as the whole temporal interval when the stance was taken by the agent.

Borrowing from UML flow charts (Seidl et al., 2012, pp. 85-106), we suggest portraying all individual events using rounded rectangles. This is as opposed to the square-edged rectangles used to portray epistemic elements. Here is a simple flow chart of a history of some theory:

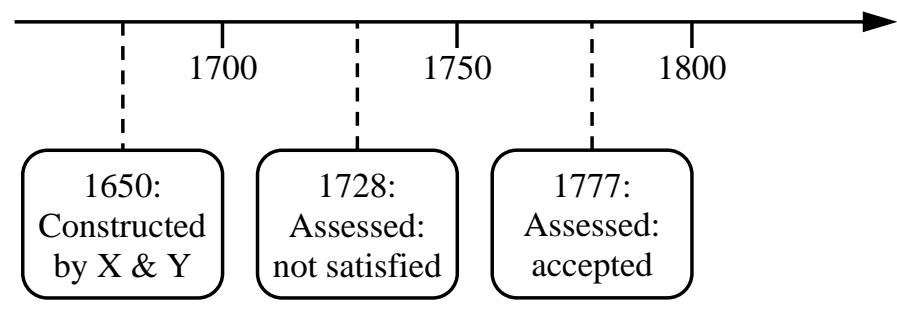

Whenever possible, we recommend showing only regular interval years on the timeline itself with the years of the respective events indicated in their event boxes.

For events that lasted for an extended period, we suggest using horizontal bars: 


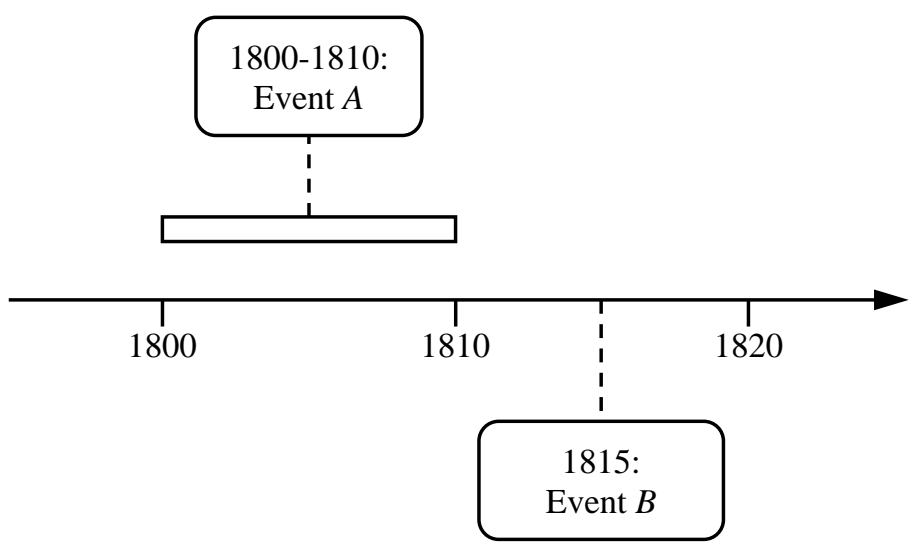

We have considered the idea of using horizontal brackets to indicate extended periods, but opted to go with rectangular period bars, as such a depiction would be inconsistent with our decision to depict all temporal intervals in horizontal period bars. Among other things, interval bars allow for more flexibility when indicating that the temporal interval in question continues beyond the period captured on the timeline. They also allow the depiction of imprecise or missing data (more on this below).

A historical timeline can depict the events that happened at individual time points and extended temporal intervals of acceptance, use, pursuit, etc.:

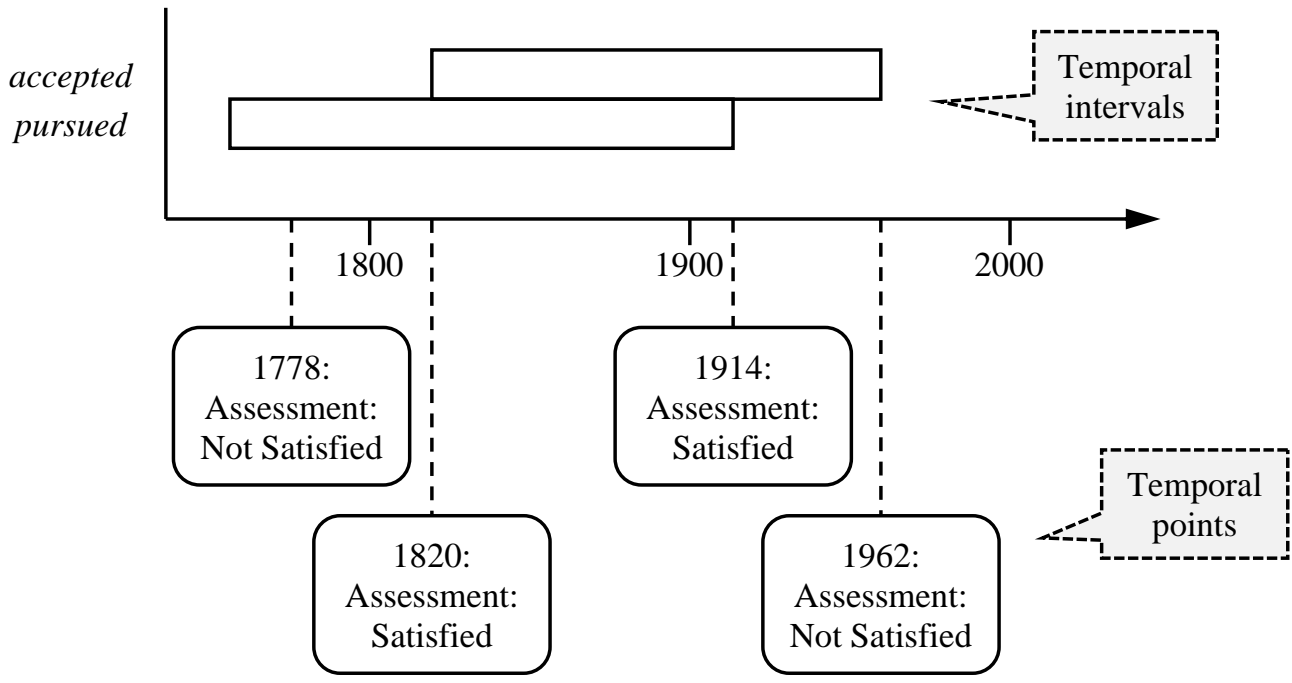

Note that all events that happened at individual temporal points are portrayed underneath the timeline; we suggest reserving the region above the timeline for interval bars for the clarity of depiction.

\section{Implicit vs. Explicit}

If we accept the suggestion of representing implicit elements with dashed bars, then the same approach can be applied to timelines. Here is what a timeline with implicit stances will look like:

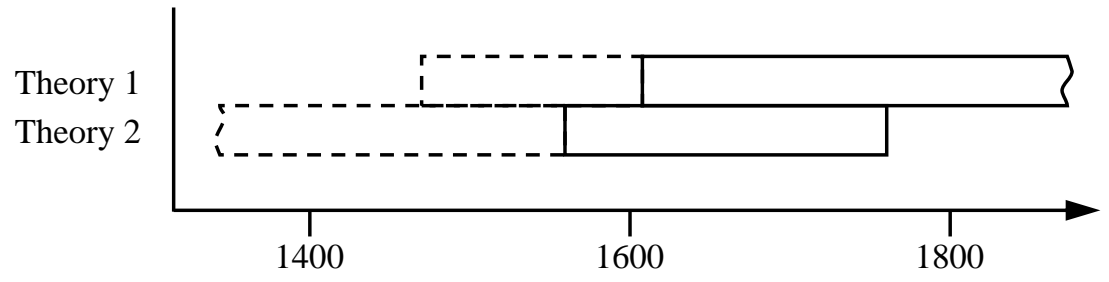


Often available historical records do not indicate a precise year of an event, but only narrow it down to a certain range. Here are some examples: "in the middle of the sixteenth century", "in the 1780s", "in the thirteenth century", "in the early 1700s", etc. To depict imprecision of the historical data on timelines, we suggest using whiskers with straight edges indicating the respective period when the acceptance or rejection took place (Barseghyan, 2021; for similar usages in other areas of knowledge visualization, see Cumming, Fidler, \& Vaux, 2007; Sarkar, 2015):

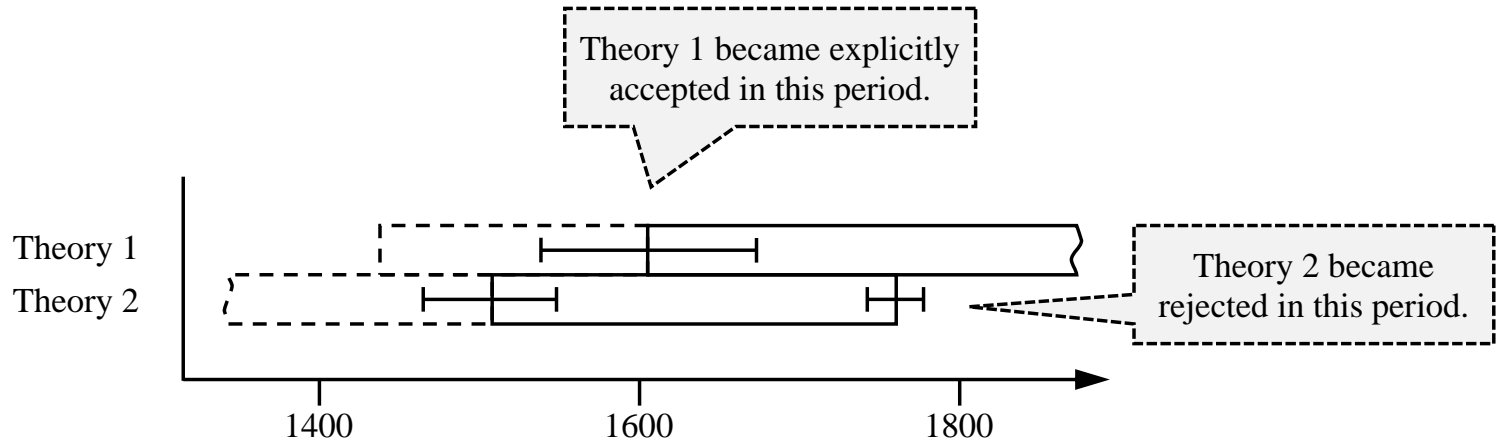

Since historical records on theory and questions acceptance and method employment are often missing or imprecise, we suspect that this visualization technique will be invaluable in highlighting such gaps and identifying possible areas of future research.

The same technique can be applied to visualizing events on timelines:

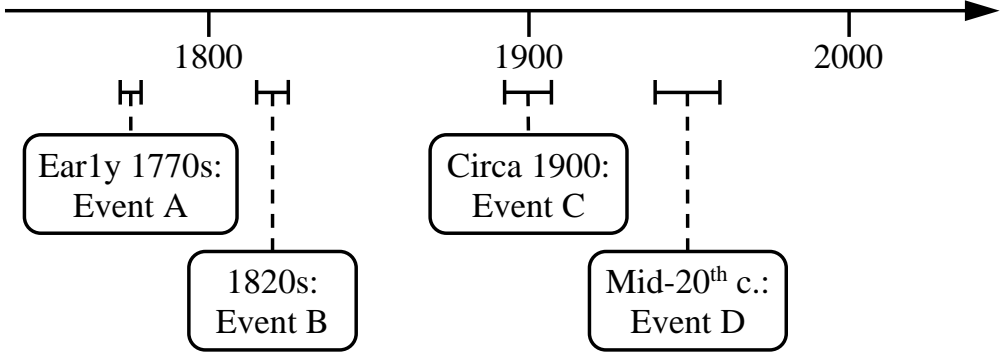

Many historical texts use the words "early", "middle", and "late" to refer to different periods within a century or a decade. There is currently no accepted demarcation when the early $20^{\text {th }}$ century ends and the mid- $20^{\text {th }}$ century starts, or when the mid- $20^{\text {th }}$ century ends and the late $20^{\text {th }}$ century starts, which means that in most cases the exact meanings of these phrases will have to be deciphered from the respective contexts. We suggest the following default meanings for these terms:

- Early: First 30\% of the period. E.g., early $19^{\text {th }}$ century $=1801-1830$, early 1920 s $=1920-1922$.

- Middle (Mid): Mid 40\% of the period. E.g., mid-19 $9^{\text {th }}$ century $=1831-1870$, mid-1920s $=1923-1926$.

- Late: Last 30\% of the period. E.g., late $19^{\text {th }}$ century $=1871-1900$, late $1920 \mathrm{~s}=1927-1929$.

We hope that with time these meanings will become standard.

At times, we know that a certain theory was accepted by an agent at a certain date, but we don't know when it became accepted or when it became rejected, i.e., we don't know the exact period of acceptance. Similarly, we often know that a theory was accepted for the entire duration of a period but have no data concerning when the acceptance or rejection took place. For instance, sometimes historical texts say that the theory was accepted by year $X$, or that it was accepted at least until year $Y$. What this tells us is that the actual acceptance period could have been longer, i.e., no claim is being made that the theory actually became accepted in year $X$, or that it became rejected in year $Y$. To visualize such situations, we suggest adding short horizontal lines at the respective ends of the acceptance bar: 


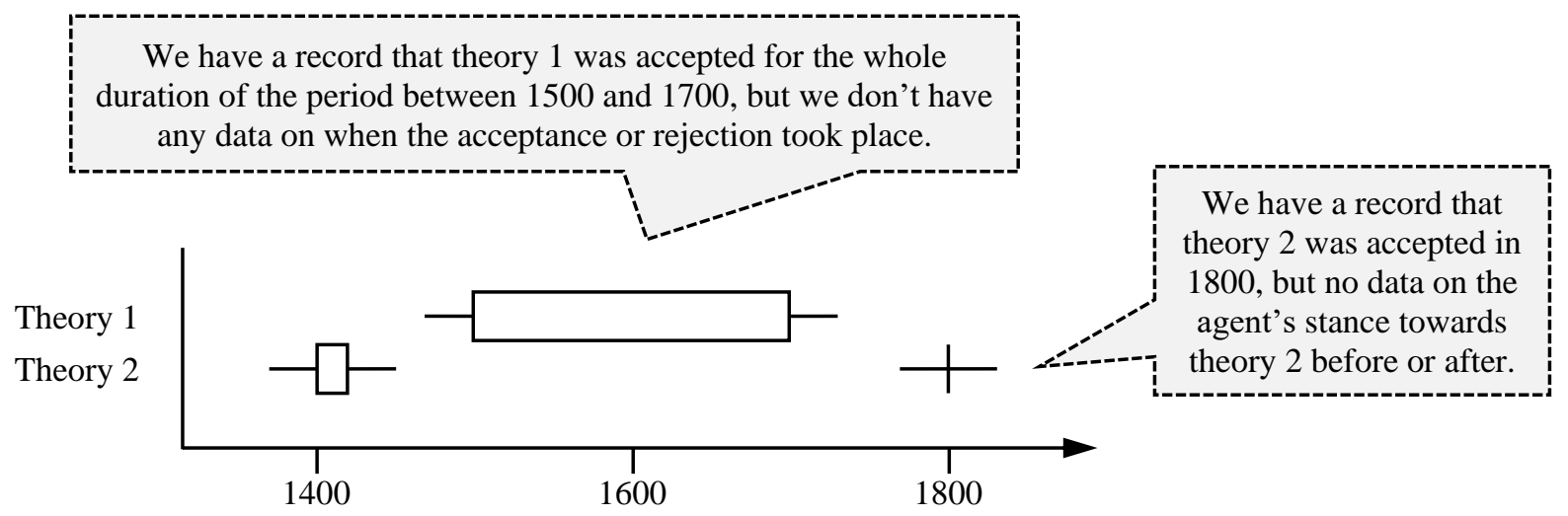

Note that the lines have a standard width equal to the height of the bar. Unlike range lines, these short lines are open ended which indicates our lack of knowledge concerning when the period in question began or/and when it ended. Once again, such a depiction highlights our lack of historical data and encourages future research.

It is also important not to confuse these horizontal lines with wavy edges: while the former indicate lack of knowledge beyond the indicated period, the latter shows that the respective period continues beyond the period captured on the timeline.

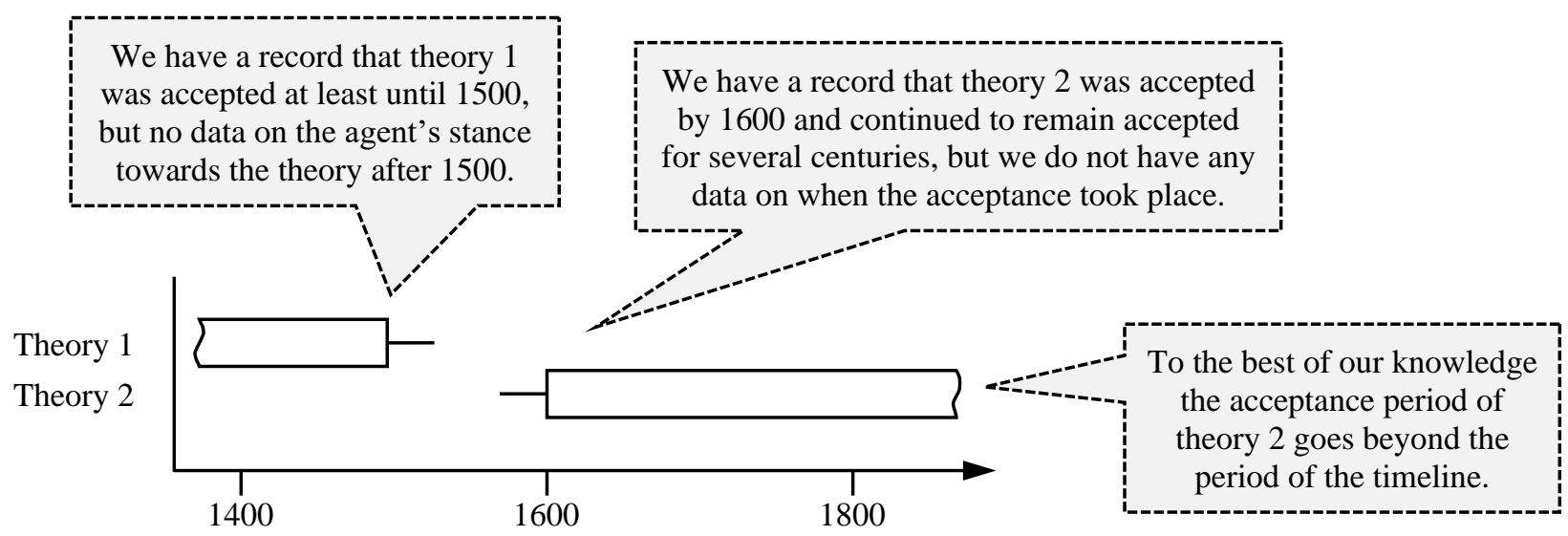

\section{Compressed Periods}

At times, it might be necessary to compress parts of a timeline, specifically, when a middle section of a timeline does not have much information to display, or when that information is not important in the given visualization context. Consider the following timeline:

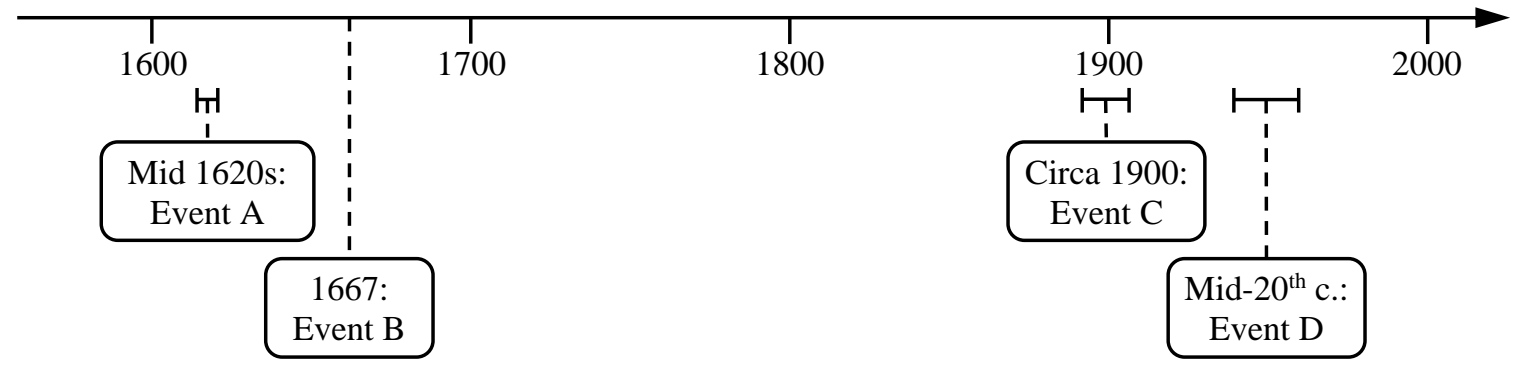

As it stands, the timeline has a huge gap in the middle section. The visual gap itself can be useful when the goal is to highlight such gaps in our historical knowledge. Yet, that is not always the goal of a timeline, in which case it might be useful to compress the middle section. To do so, we suggest jagging (zigzagging) compressed sections: 


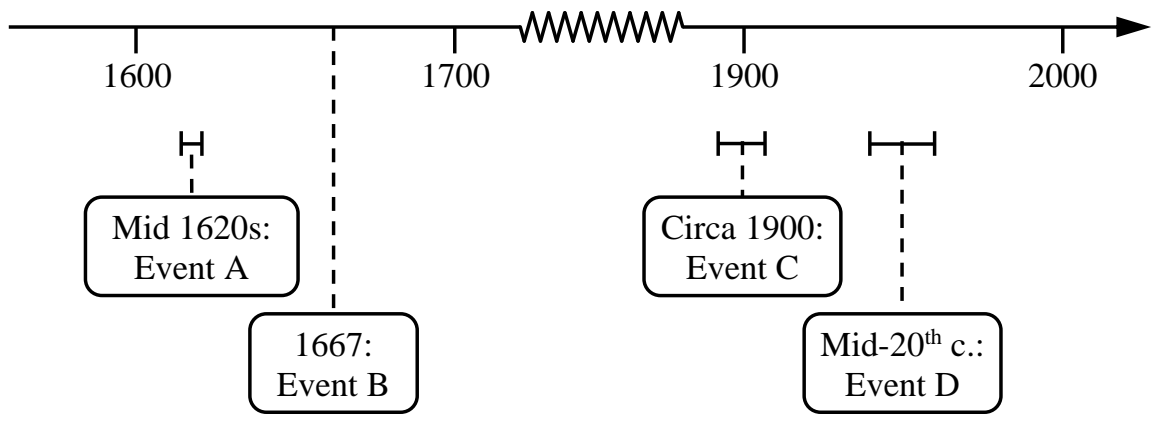

Compressed timelines might be also useful for illustrating the beginning and the end of a certain period of acceptance or pursuit while skipping the middle part. Here is an example:

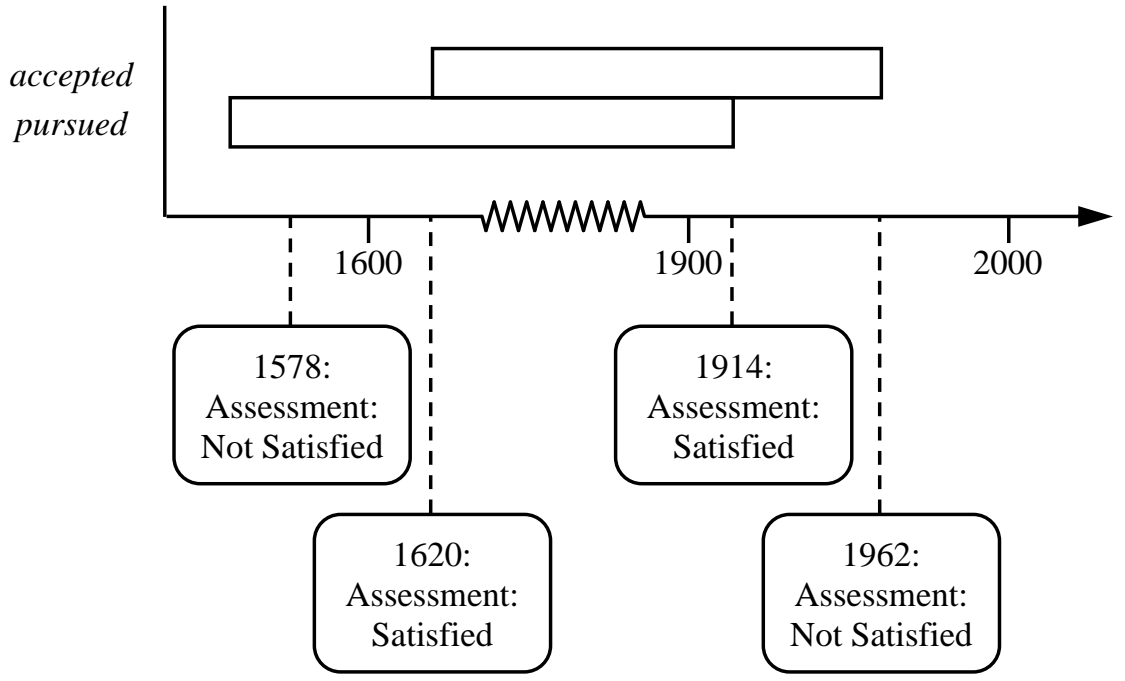

\section{Indicators}

Each historical record can have any number of historical indicators, i.e., evidence supporting that record. The list of potential indicators includes primary and secondary sources, encyclopedias, textbooks, etc. Thus, one question is whether we need any diagrammatic way of portraying a record's indicators, or whether indicators should be treated more like citations of relevant sources and remain purely textual. Our current consensus is that including historical indicators (such as quotes and citations) en masse would inevitably convolute our diagrams. Thus, we believe that, as a rule, they are best given in the text itself as well as in appendices and footnotes, if necessary. Occasionally, it may be appropriate to include, say, a fragment of the original source text in a callout to make it easier for the reader to compare the diagrammatic representation with the original text, but such instances should be kept to a reasonable minimum, for otherwise there is a serious risk of cluttering the diagrams. On web diagrams, a simple pop-out with the respective indicators would be sufficient.

\section{Epistemic Agents}

To depict an individual epistemic agent, we use the standard UML symbol for actors with an added label stating the name of the agent:

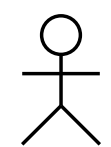

Galileo

Galilei

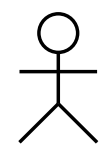

Albert

Einstein 
While it might be tempting to use a separate symbol for communal agents, we believe that no such specific symbol is necessary. Following the standard usage of a stick figure in UML, we suggest using the same symbol for all epistemic agents, individual or communal. The difference between the two should be obvious from the respective accompanying labels:

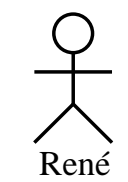

Descartes
Paris Natural

Philosophy Community

Communal agents can consist of other communities and individuals. One way of depicting the relations between communities, subcommunities, and individuals, is by linking the respective agent symbols by the standard UML connector for generalization:

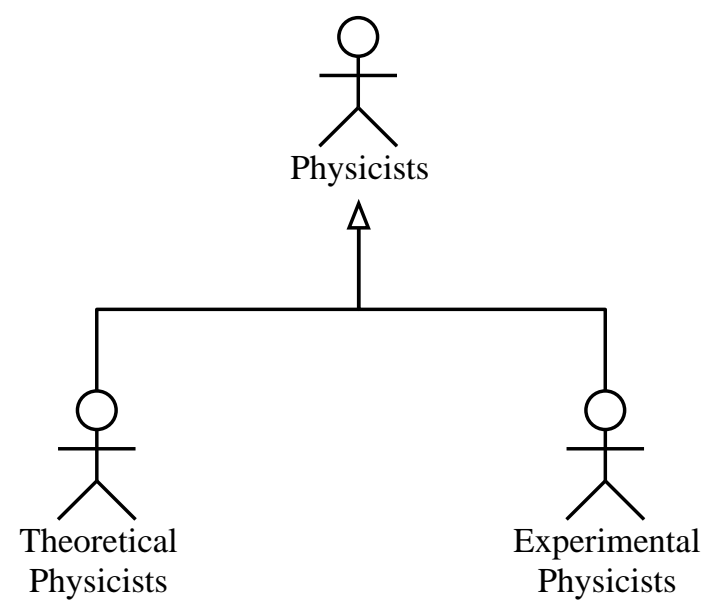

Since the agent symbol is compact, such depictions can come in handy on diagrams where many agents should be included simultaneously.

Another common relation that holds between agents is that of authority delegation, normally depicted by means of a solid line connecting the delegating agent with the expert agent, where the line culminates with a white box connected to the expert. While authority delegation relations are commonly visualized by connecting the respective mosaics (see section Error! Reference source not found. below), they can also be depicted by using the respective agent symbols. Here is, for instance, a depiction of the authority delegation between two agents on a certain topic:

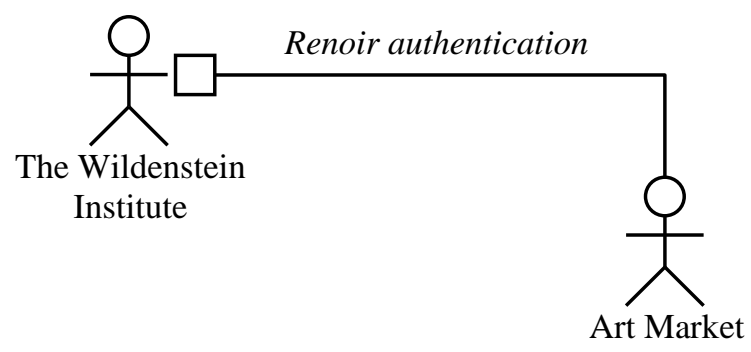

This option can become handy on complex authority delegation diagrams involving many epistemic agents. Another acceptable means of depicting relationships of authority delegation between agents is detailed below (see section Error! Reference source not found.). 


\section{Epistemic Collections}

We have experimented with various means of depicting mosaics (e.g., Barseghyan, 2015, p. 193). One natural suggestion is to use the standard UML symbol for package, which looks like a folder:

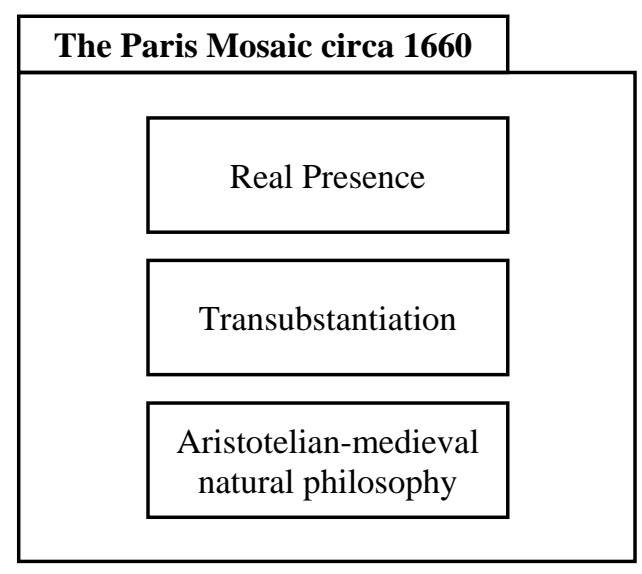

In this representation, the title of the folder is either the name of an epistemic agent that is the bearer of the mosaic in question or of the mosaic itself, with the agent that bears it specified textually. In this case, it is the community of natural philosophers in Paris, circa 1660. The boxes within the folder indicate salient epistemic elements that are constituent parts of the agent's mosaic, such as, in this case, the "Real Presence", the belief that Jesus Christ is really present in the bread and wine of the Christian sacrament of the Holy Eucharist (adapted from Barseghyan, 2015, p. 193).

When deemed necessary, epistemic elements of different types (e.g., question, theories, methods) can be grouped together as constituents of the same mosaic. In such cases, a dashed separator can be used to distinguish elements of different types. In the following example theories are depicted on the left, with a method depicted on the right:

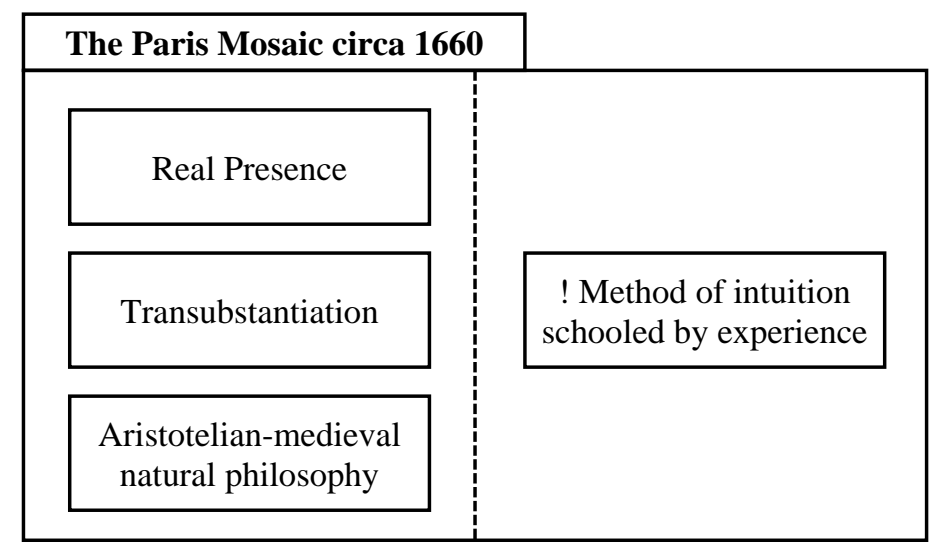

Color highlighting has been often used in scientonomic representations. Such highlighting is still acceptable, since it doesn't convey any semantic information not conveyed by other means (see section Highlighting and Callouts below). Thus, when desired, the title and the body of a mosaic folder can be colored accordingly for aesthetic purposes: 


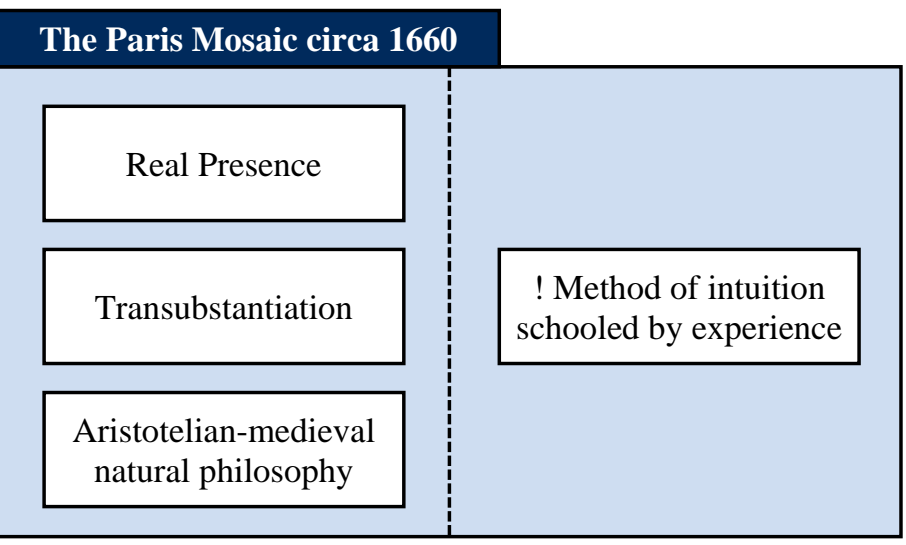

There are several types of relations between mosaics that are currently accepted in scientonomy. When comparing and contrasting the content of two or more mosaics, it might be useful to portray the two mosaics next to each other to make it clear where the respective agents agreed or disagreed with each other. The following diagram shows two key points of contention between Catholic theology and Aristotle's system:

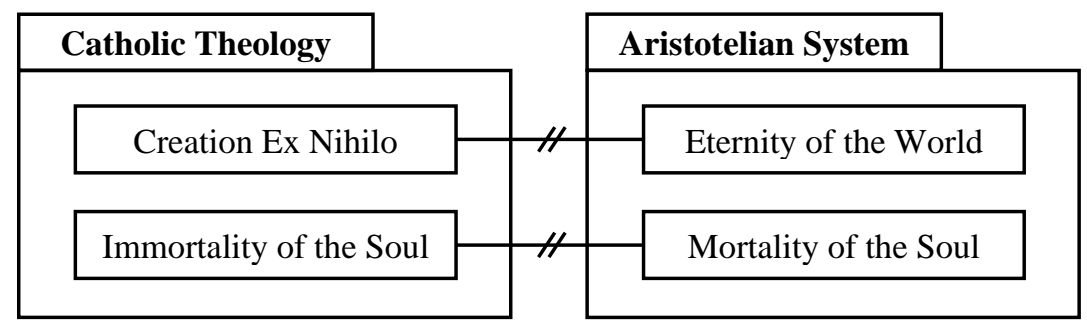

The conflict between two or more mosaics can be demonstrated also by omitting the details:

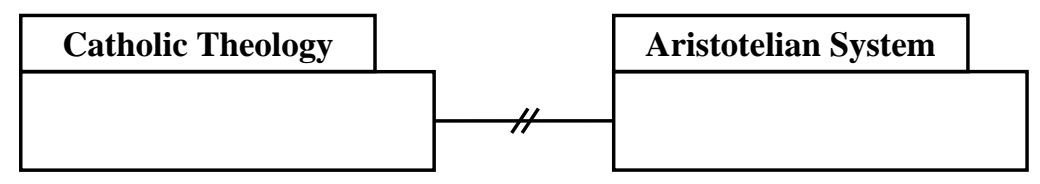

At times it might be necessary to indicate not only the disagreement between the two mosaics but also to visualize their overlaps. In such cases, we recommend using overlapping folders and positioning the shared elements in the overlapping area. Here is an example:

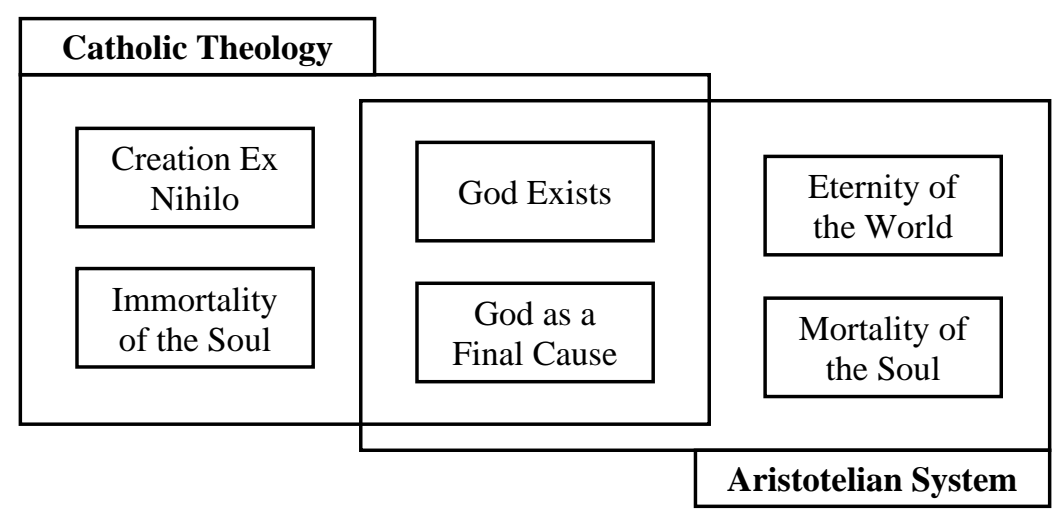


Here is the same diagram with a highlighted overlap:

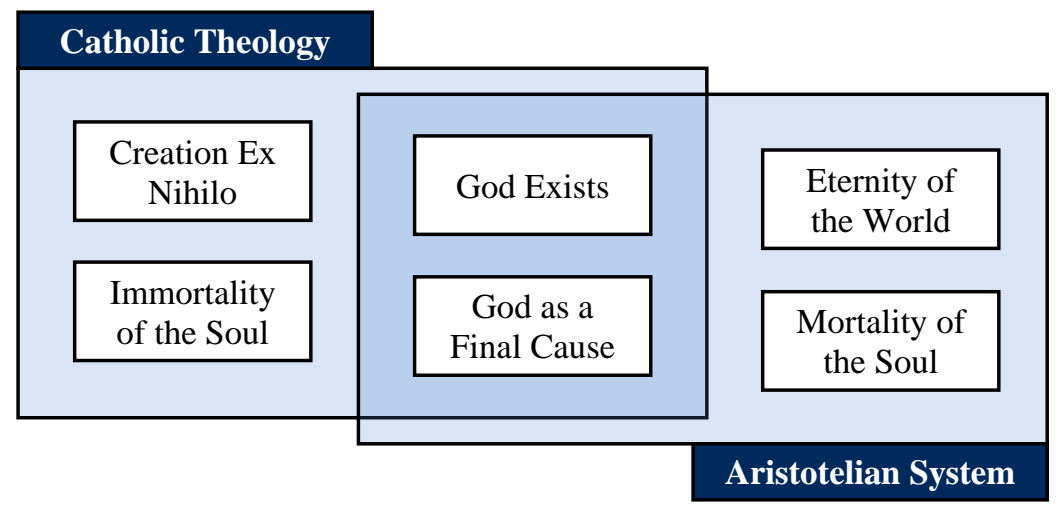

The same approach can be effectively used to portray the relations between three or even four mosaics. The following diagram demonstrates three mosaics with both shared and unshared elements:

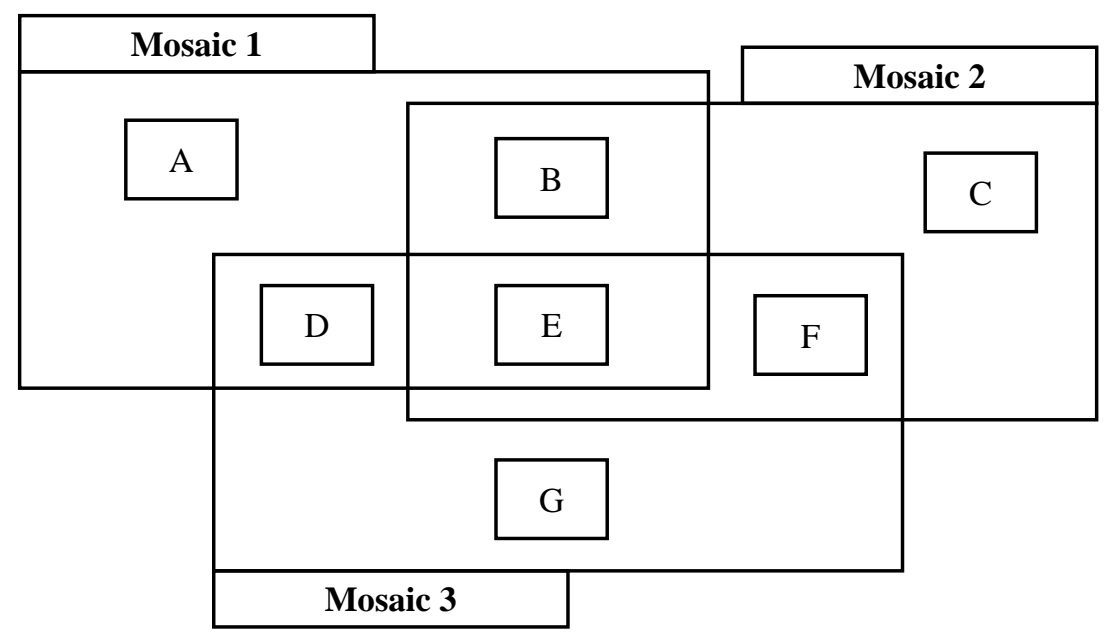

Above, we discussed the relationship of authority delegation, that can hold between agents. As noted above, it can be represented diagrammatically by a solid line with a white box at the end. The same connector can be used when linking the respective mosaics of the two agents. Here is an example of a diagram showing that the art market community delegates authority on authenticating the works of Claude Monet to the Wildenstein Institute (adapted from Loiselle, 2017, p. 48):

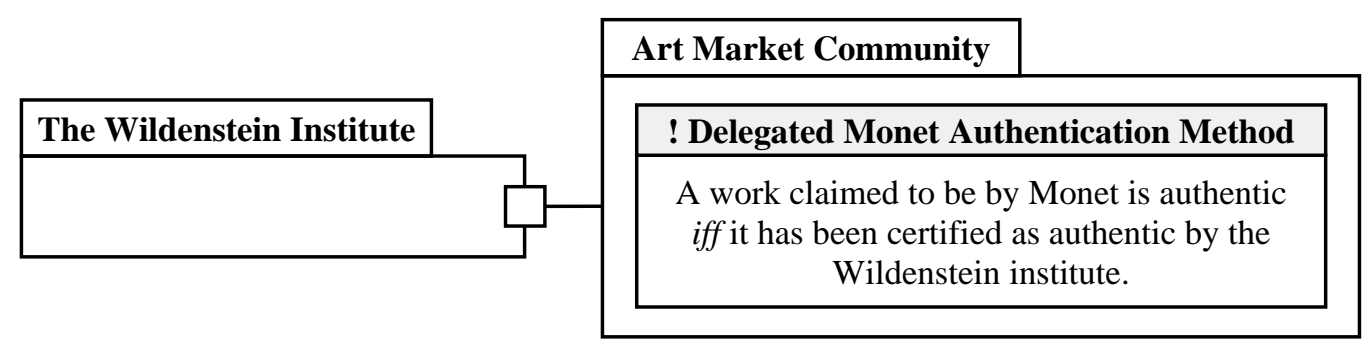

Rather than the stick figures discussed above, both agents involved in the authority delegation relationship are represented by their respective mosaics, which allows for the depiction of the boxes representing salient epistemic elements accepted within the agents' mosaics. In this case, the theory that forms the basis for the art market community's authority delegation to the Wildenstein Institute for authentication of the works of Monet is represented. 
Here is a highlighted version of the same diagram:

\section{Art Market Community}

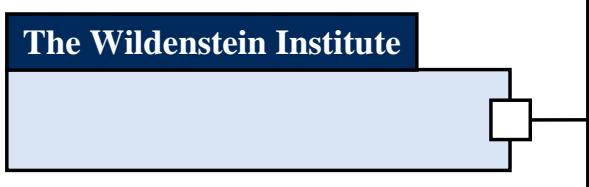

! Delegated Monet Authentication Method

A work claimed to be by Monet is authentic iff it has been certified as authentic by the Wildenstein institute.

When the formulation of the delegated method is omitted, it is recommended to indicate the topic of delegation on the connector itself, thus:

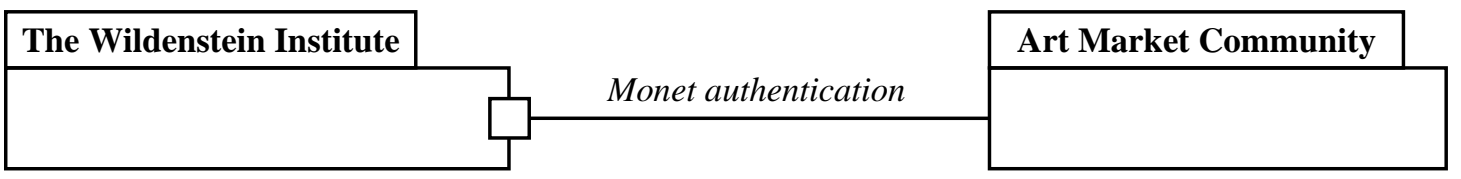

When authority on a certain topic is delegated to more than one agent, often such authority delegation is hierarchical, where different experts are delegated different degrees of authority over a certain topic (Loiselle, 2017). Traditionally, we have depicted hierarchical authority delegation by indicating the order of experts in the respective authority delegation boxes. Here is an example (adapted from Loiselle, 2017, p. 47):

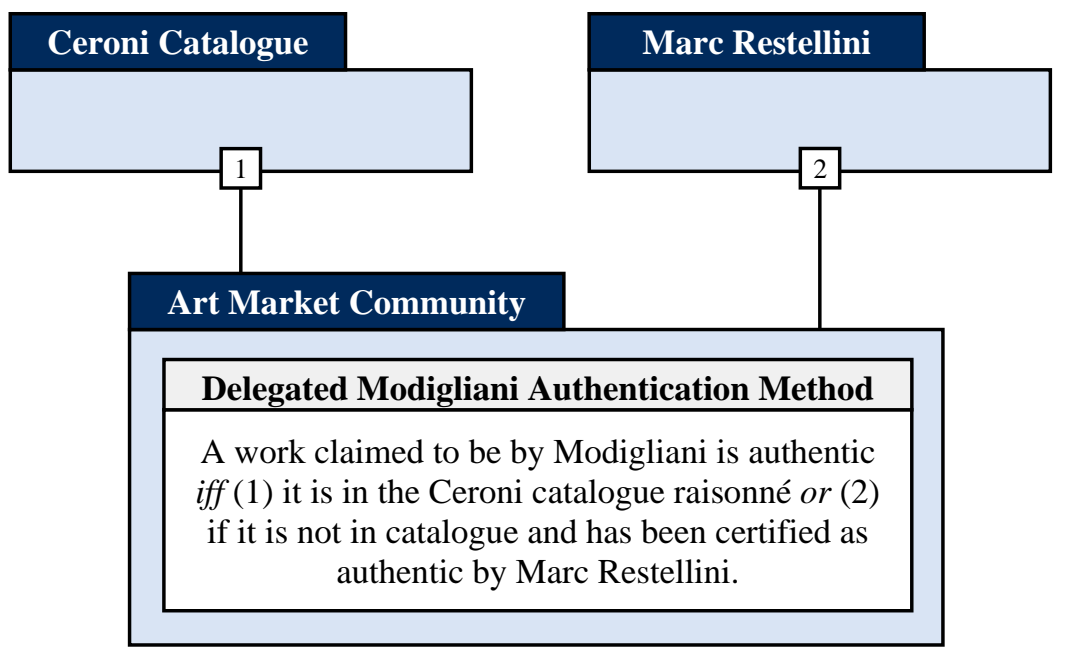

\section{Additional Visualization Techniques}

In addition to visualization tools for depicting the entities and relations found in the scientonomic ontology, we also use a few more general-purpose visualization techniques.

\section{Highlighting and Callouts}

Sometimes it might be necessary to highlight, emphasize, or explain certain important aspects of the diagram. One straightforward (and accessibility-friendly) way of doing this is by using callouts. As is usual in scientonomic practice, rectangular callouts with a dotted outline and slightly shaded background can be used. Many of the diagrams in this paper contain such callouts. Callouts can be pointing to one or more diagrammatic elements. Here is an example of a callout pointing to two elements: 


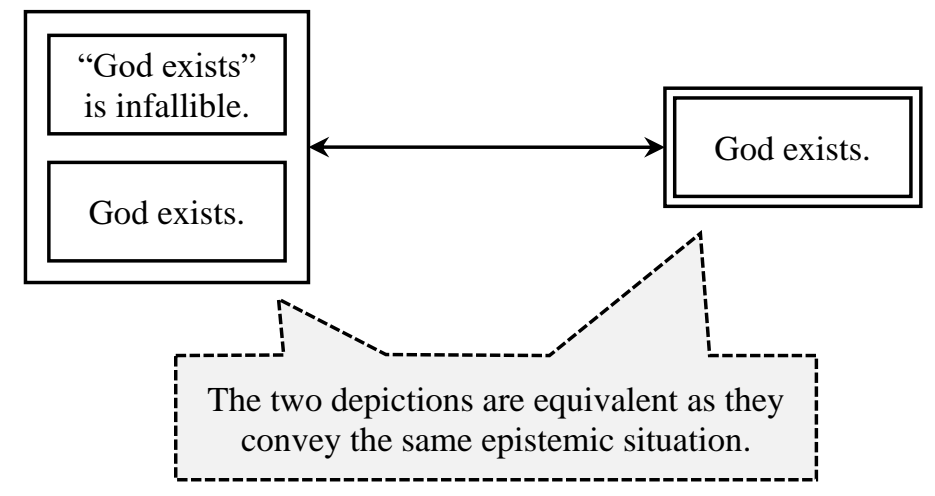

As with any diagrammatic element, callouts are to be introduced with moderation to keep the clutter at an aesthetically acceptable minimum and ensure that the readability of the diagram is not impaired.

Another accessibility-friendly way of highlighting the elements of a diagram is color inversion. For example, in some contexts, it might be necessary to visually distinguish between the axioms of a certain belief system and the theorems which follow from these axioms. In such cases, the title of an epistemic element can be inverted to highlight that element. This technique might be useful for highlighting the starting points of a belief system - a feature that can come in very handy in practice (e.g., when trying to identify the axiomatic premises of a certain political argument). In scientonomy, such inversions have been used to highlight the laws of scientific change (see for example, Barseghyan, 2015, pp. 123, 128, 168, 173; Barseghyan \& Levesley, 2021, p. 11). The following diagram highlights the fact that the law of theory demarcation is being suggested by the authors as an axiom (Fraser \& Sarwar, 2018, p. 76):

\section{The Law of Theory Demarcation}

If a theory satisfies the demarcation criteria of the method employed at the time, it becomes scientific; if it does not, it remains unscientific; if assessment is inconclusive, the theory's status can become scientific, unscientific, or uncertain.

\section{Theory Acceptance $\equiv$}

An accepted theory is a scientific theory that is taken as the best available description or prescription of its object.

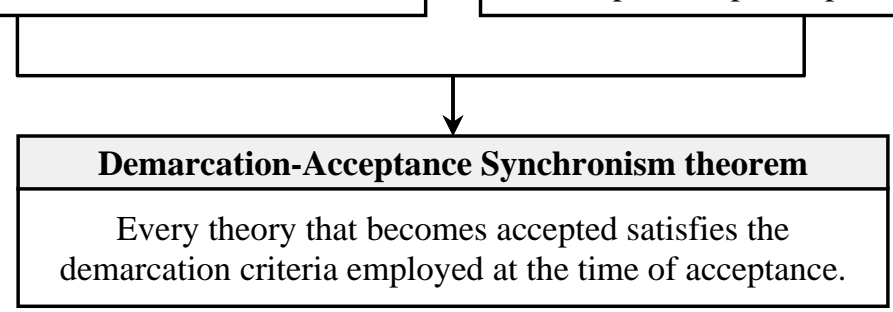

Note that there is currently no scientonomic distinction between axioms and theorems, and thus no basis for stipulating the corresponding visualization. Thus, such highlighting is advisable only when the epistemic agent in question clearly accepts a distinction between axioms and theorems, and there exists a special need to highlight the distinction. This means that the practice of highlighting scientonomic axioms can be maintained, but there is no mandatory requirement to highlight the axioms at all times. Thus, any highlighting of axioms is completely optional, i.e., the following depiction would be perfectly acceptable: 


\section{The Law of Theory Demarcation}

If a theory satisfies the demarcation criteria of the method employed at the time, it becomes scientific; if

it does not, it remains unscientific; if assessment is inconclusive, the theory's status can become scientific, unscientific, or uncertain.

\section{Theory Acceptance $\equiv$}

An accepted theory is a scientific theory that is taken as the best available description or prescription of its object.

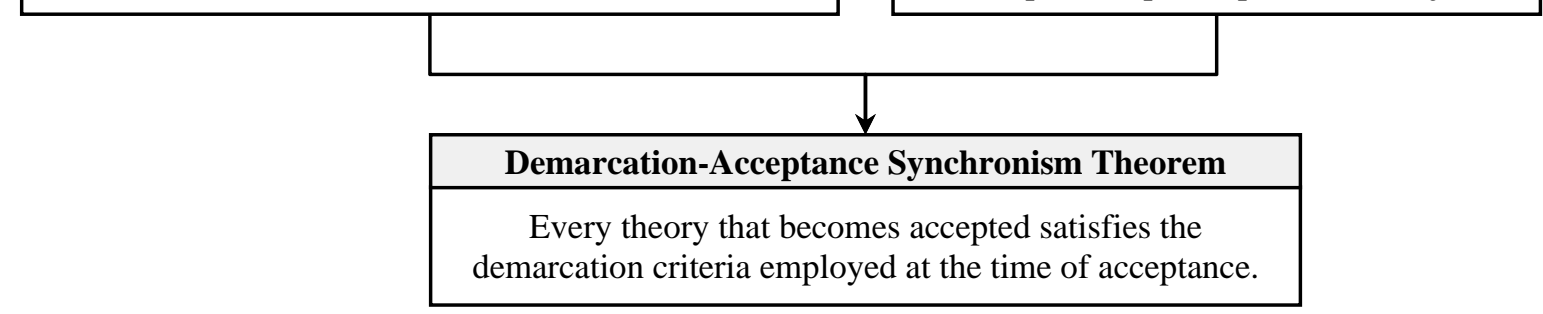

In addition, different time periods can be highlighted to sharpen the difference between different theories, different epistemic stances, etc. Once again, this is allowed only if such a highlighting does not exclusively convey any semantic information not conveyed by any other standard element:

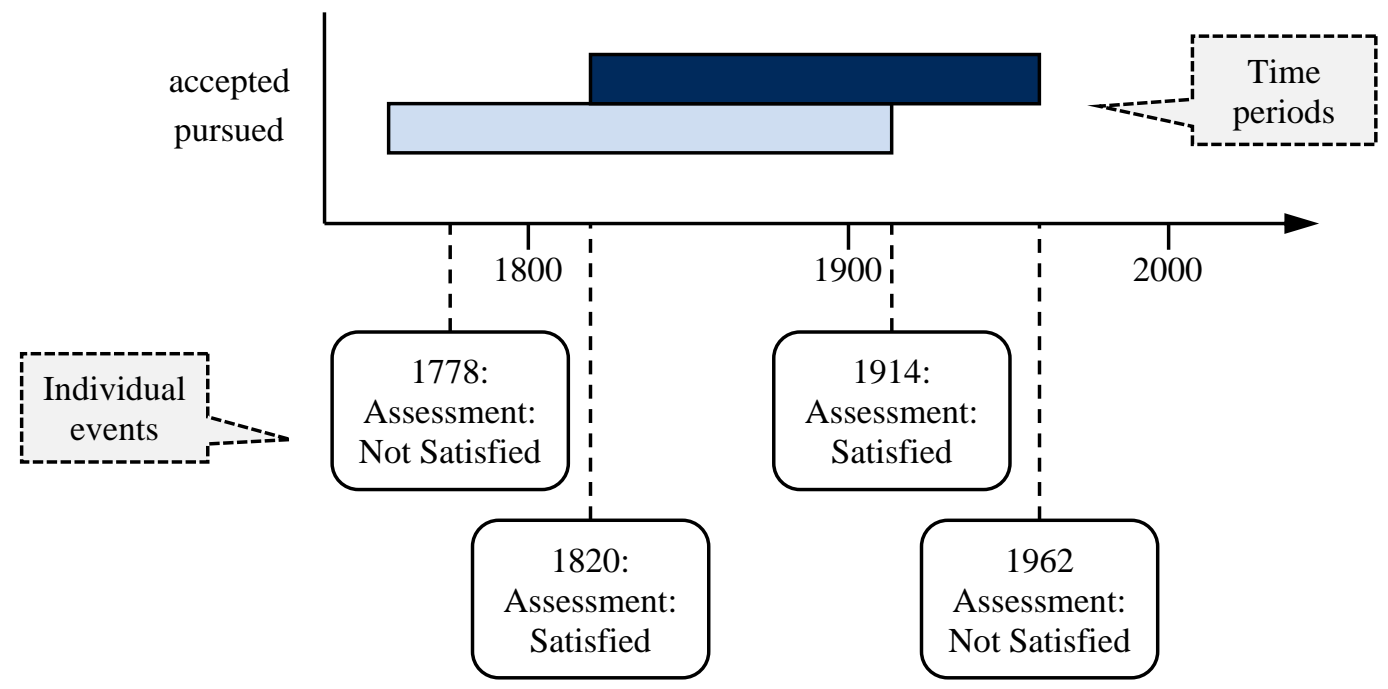

\section{Influences}

Any historical narrative inevitably speaks of the influences that agents exert on one another (and also possibly of influences between mosaics, or between elements). In scientonomic diagrams, temporal influences of this sort have been portrayed by lines with stealth/notched arrowheads. This is problematic, since this type of arrowhead is then used to visualize two distinct relations - the epistemic (atemporal) relation of implication (e.g.,

Microsoft and Google are using slightly different taxonomies of arrowheads. What is known as a stealth arrowhead in MS Word and MS Power Point is called notched in Google Docs and Google Slides.

$B$ follows from $A$, or $A$ is a reason for $B$ ) and the temporal relation of influence (e.g., $B$ causes $A$, or $B$ influences $A$ ). Of course, this may not necessarily be a problem, as the two usages rarely appear in the same diagram owing to the fact that epistemic relations are portrayed on synchronic diagrams, while causal relations are portrayed on diachronic diagrams. Yet, we can envisage situations where the two might be combined in the same diagram, e.g., when it is necessary to show both the content of the two mosaics (with some implication relations) and the fact that one mosaic influenced the other. To avoid confusion, therefore, we suggest distinguishing the two types of relations by means of the respective arrowheads. We will reserve the stealth/notched arrowhead only for epistemic relations and will use the open arrowhead only for influence relations. 

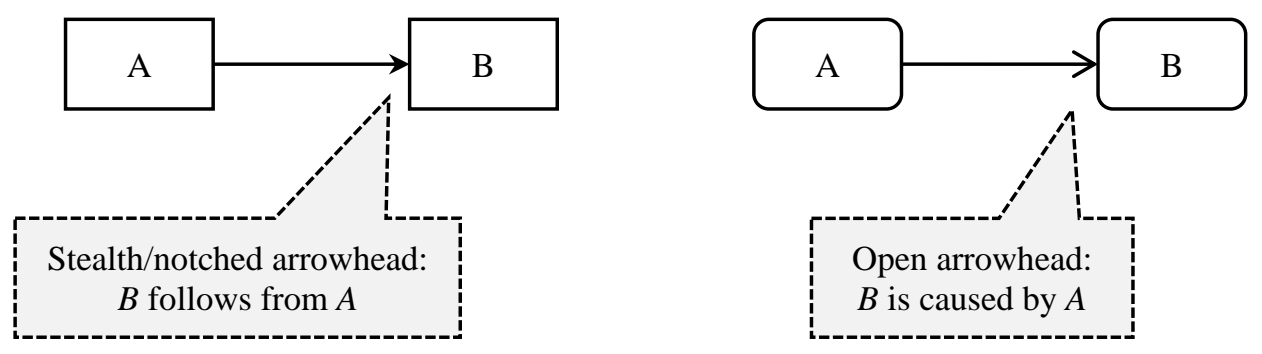

Deterministic causal influences will be depicted by means of a solid line with an open arrowhead. Probabilistic causal influences will be depicted by a branching dashed line with an open arrowhead. Finally, a dashed line with an open arrowhead will be used for all other generic types of influences (normally expressed in literature through such verbs as "influenced", "shaped", "contributed to", etc.):

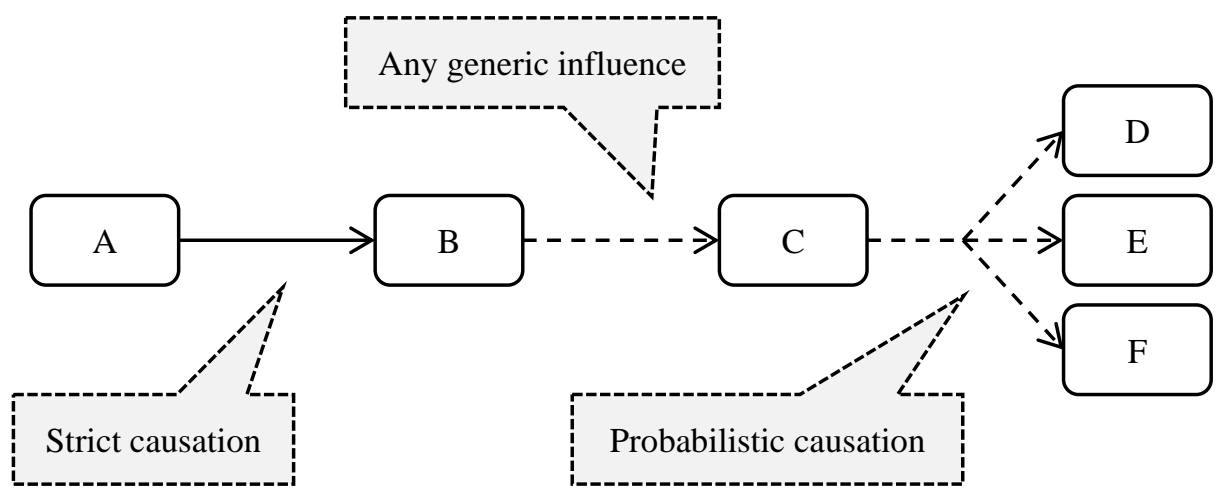

Unlike epistemic relations, we suggest not to elbow the influence connectors, but use straight or branching connector lines instead. Here is an example of a diagram involving both deterministic and probabilistic influences (adapted from Fraser \& Sarwar, 2018, p. 73):

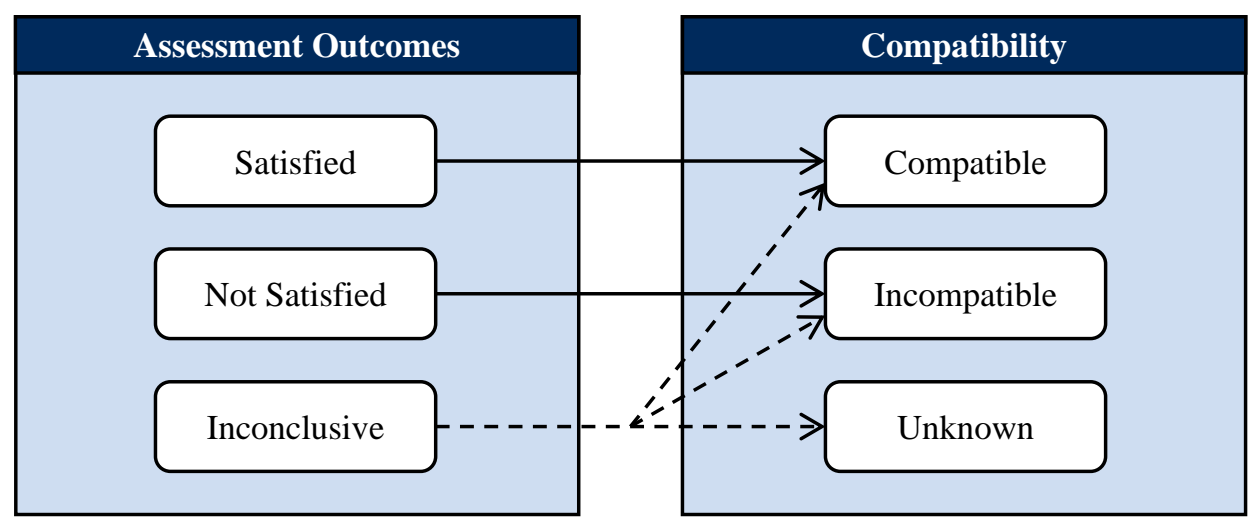

The diagram visualizes the content of the law of compatibility suggested by Fraser and Sarwar. It shows that if a pair of elements is judged to be satisfying the respective compatibility criteria employed, the pair becomes to be considered compatible within the mosaic; if it is not, it is deemed incompatible; and if assessment is inconclusive, the pair can become compatible, incompatible, or its status may be unknown. Note that instead of spelling out the comprehensive descriptions of the events, the diagram only uses the titles of the events and the respective influence connectors for succinctness.

It is also possible to indicate that an event does not influence another event, or that a certain state of affairs is impossible given some other state of affairs. 


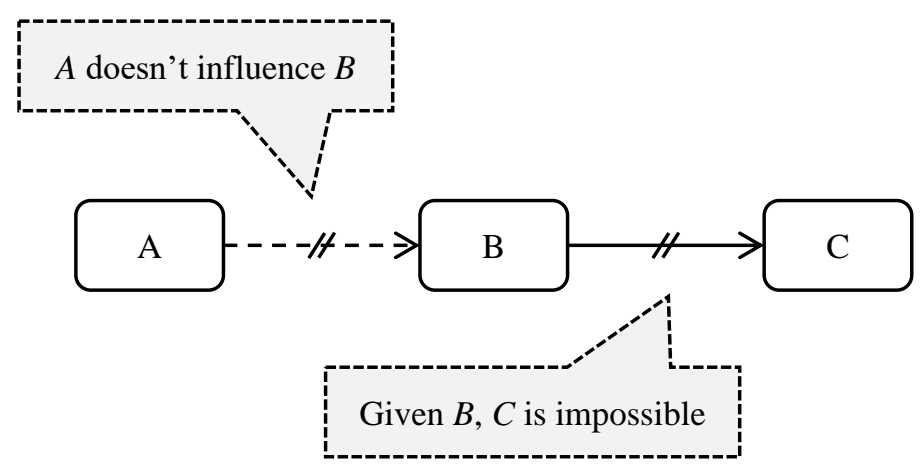

Here is an example of the latter scenario (adapted from Patton, Overgaard, \& Barseghyan, 2017, p. 34):

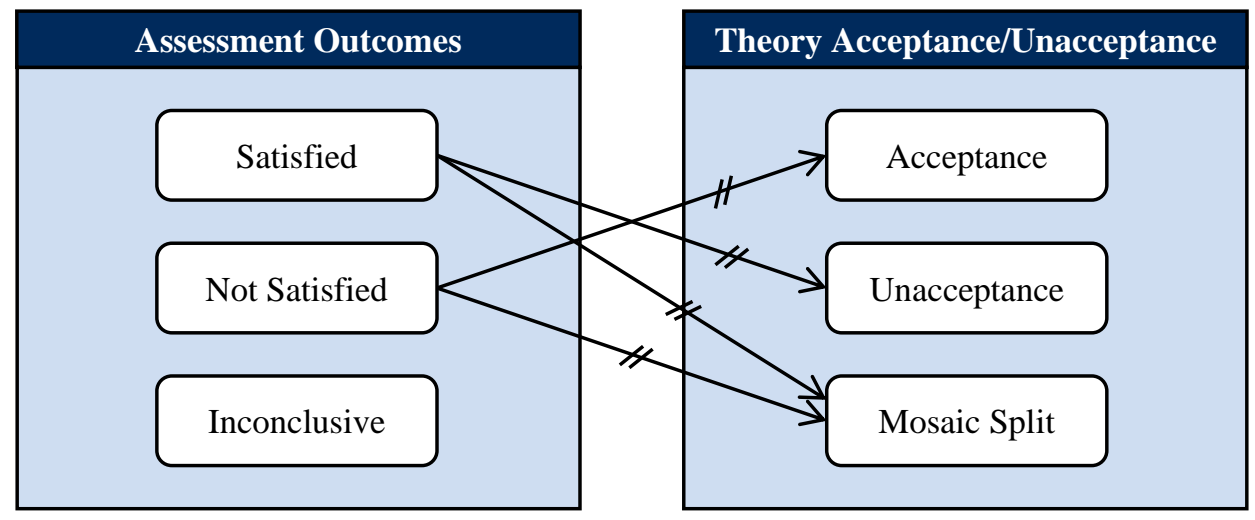

The influence connectors can be also used to depict the influence of epistemic agents on each other:

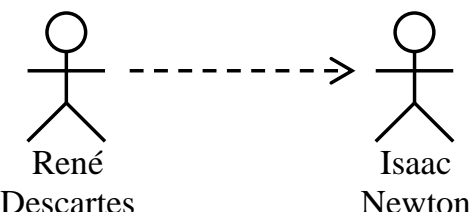

In principle, the influence connectors can be used to depict any temporal influences of agents on other agents. In most cases, the influences between agents will be of some non-deterministic kind and, thus, they will be depicted by dashed arrows. This can give the impression that all influences between agents carry equal weight, which might be deceiving. Ultimately, it may be useful to depict different types of influence by different lines/arrows, but this is a task for future theoretical research. Meanwhile, the nature of a specific non-deterministic influence can be indicated by means of an accompanying label, like in the diagram below:

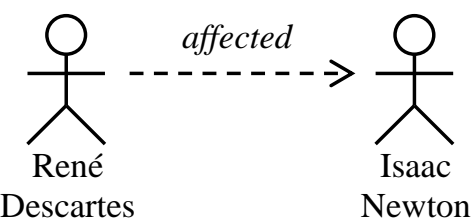

\section{Grouping}

In might sometimes be important to group various elements of a diagram. A regular rectangle with a title box can be used for this purpose. Here is an example that we have previously discussed showing the grouping of the variety of assessment outcomes (adapted from Patton, Overgaard, \& Barseghyan, 2017, p. 31): 
Satisfied

Not Satisfied

Inconclusive

Note that such groupings are different from epistemic collections (such as, mosaics) depicted as folders. Groupings are more generic in nature, as they do not necessarily concern epistemic elements or stances taken towards them by epistemic agents. In principle, there are no limitations on how such groupings can be used, other than those concerning readability and aesthetic appeal of the diagrams.

\section{Diagram Captions}

When a diagram is incorporated in a text, it can be accompanied by a caption. How exactly the caption is formatted depends on the respective style (APA, MLA, Chicago, etc.). Thus, our diagrammatic notation cannot impose any specific requirements here.

\section{Other Attributes}

It might be tempting to populate the diagrams with additional information such as the theory's year of construction, the theory's authors, bibliographic information etc. However, we strongly recommend refraining from such practice as it would make the diagrams too convoluted. When absolutely necessary, such background information can be provided in a callout; otherwise, it's best provided I the accompanying text.

\section{A Taxonomy of Diagrams}

The visualization tools presented above can, in principle, be combined in a variety of creative ways producing many specific types of diagrams. Since diagraming can have various aims, many interrelated considerations go

Among other things, it is essential for a diagrammatic visualization to maintain a balance between informativeness and aesthetic appeal. While it might be tempting to make the diagram more informative by including more diagrammatic elements, it is important not to overcrowd diagrams. Although this may not be an issue in an electronic format where large complex diagrams can be easily handled by means of zooming and scrolling, in printed media it is advisable to refrain from depicting more than twenty titleonly epistemic elements or more than a dozen title-and-body epistemic elements in the same diagram. Obviously, this is not a hard rule; each medium will impose its specific limitations.

important to visualize. Here, we will present several types of diagrams that have proven useful for depicting the content and evolution of various belief systems.

Diagrams dealing with belief systems can be arranged into two broad categories:

- Synchronic diagrams: diagrams depicting a certain epistemic situation at a particular point in time.

- Diachronic diagrams: diagrams depicting the temporal evolution of belief systems.

In this section, we outline a variety of synchronic and diachronic diagrams by highlighting their advantages and limitations. into choosing the diagram type appropriate to a specific visualization. We do not believe that there is any one canonical visual representation that is best for visualizing a particular belief system. In fact, as our own diagramming practice confirms, any belief system can be visualized in a potentially endless variety of ways. Thus, the users of this notation can use these diagrammatic tools to construct diagrams of various types, as well as come up with their own diagram types, depending on which aspects of the belief system in question they find 


\section{Synchronic Diagrams}

Synchronic diagrams depict an epistemic situation at a particular point in time. While these diagrams can be used to visualize an agent's belief system over a long period of time (provided that the belief system was relatively stable over that period), they are not equipped to show the evolution of the agent's views. The main purpose of synchronic diagrams is to give a snapshot a certain epistemic situation. Among the most useful types of synchronic diagrams are taxonomy diagrams, theory relation diagrams, question-theory diagrams, mosaic comparison diagrams, and agent (or mosaic) relation diagrams. We will discuss these in turn.

\section{Taxonomy Diagram}

When analyzing an agent's belief system, it is often advisable to start by extracting the agent's vocabulary, i.e., their taxonomy of concepts and the relations between them. Taxonomy diagrams are a powerful tool for depicting the intricate relationships between different terms in an agent's taxonomy. These diagrams normally visualize the relations of generalization/inheritance, composition, aggregation, and/or use. In a nutshell, a taxonomy diagram is a simplified version of a standard UML class diagram; it depicts the same basic relations but omits the details that are irrelevant within the domain of belief visualization.

A minimalistic taxonomy diagram can focus exclusively on generalization/inheritance relations and show a concept with its sub-concepts, as in the following diagram depicting the three subtypes of theory:

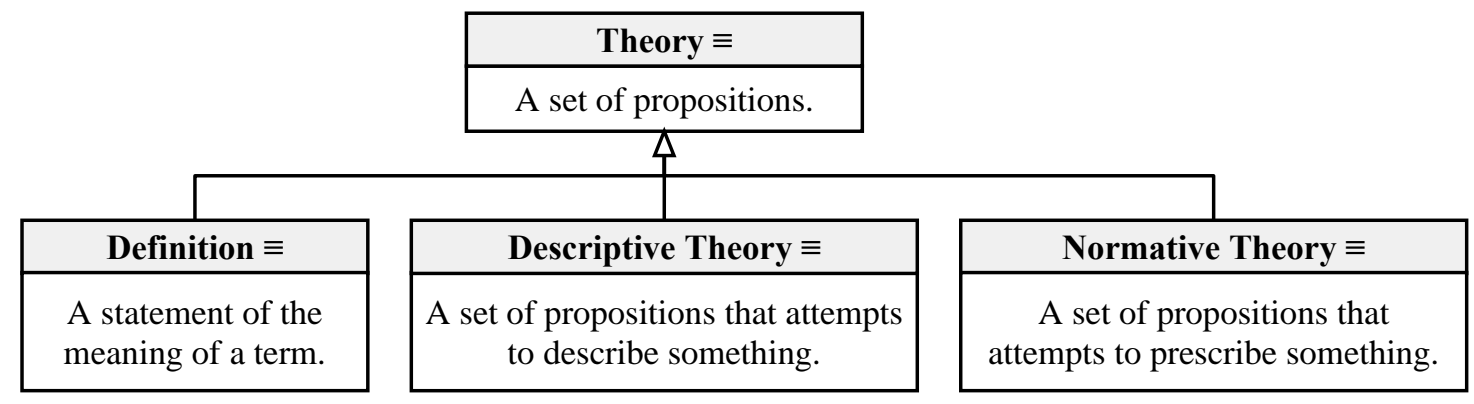

If necessary, the respective definitions of the concepts can be omitted to make space for more concepts. The following diagram portrays a fragment of our taxonomy of diagrams by skipping the respective diagram definitions:

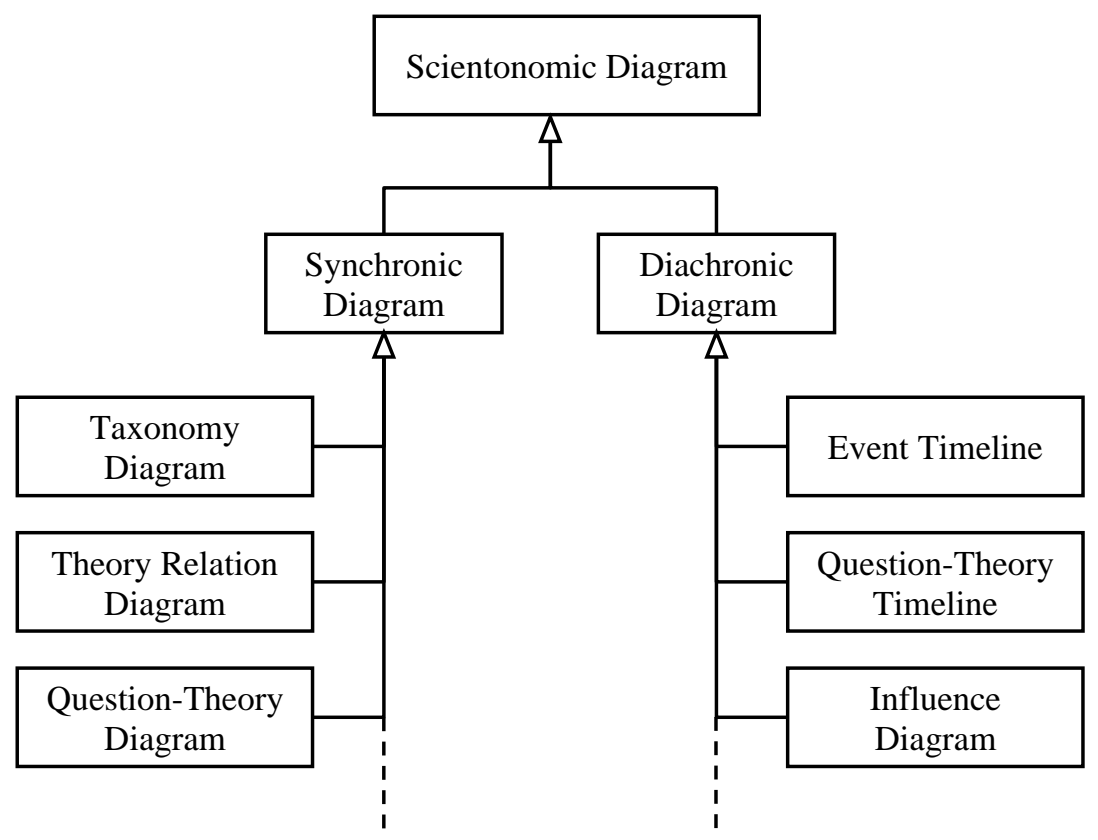


Note that since this diagram includes only concepts and since these concepts are depicted in the title-only mode, we have omitted the triple bar symbol of definition $(\equiv)$ to avoid unnecessary clutter.

Importantly, taxonomy diagrams can include not only the elements of the respective agent's ontology, but also definitions of those concepts which the agents did not believe to refer to anything existing. This is because agents can have concepts even for those entities and relations the existence of which they do not accept. For example, we have a concept of centaur, a creature that is half human and half horse, but we do not think there are actual centaurs out there. Thus, the concept of centaur is not part of our ontology, but it is still part of our vocabulary. The following diagram shows a fragment of the vocabulary shared by many French chemists in the 1780 s, including those who did not accept the existence of phlogiston or caloric (Levesley \& Barseghyan, forthcoming):

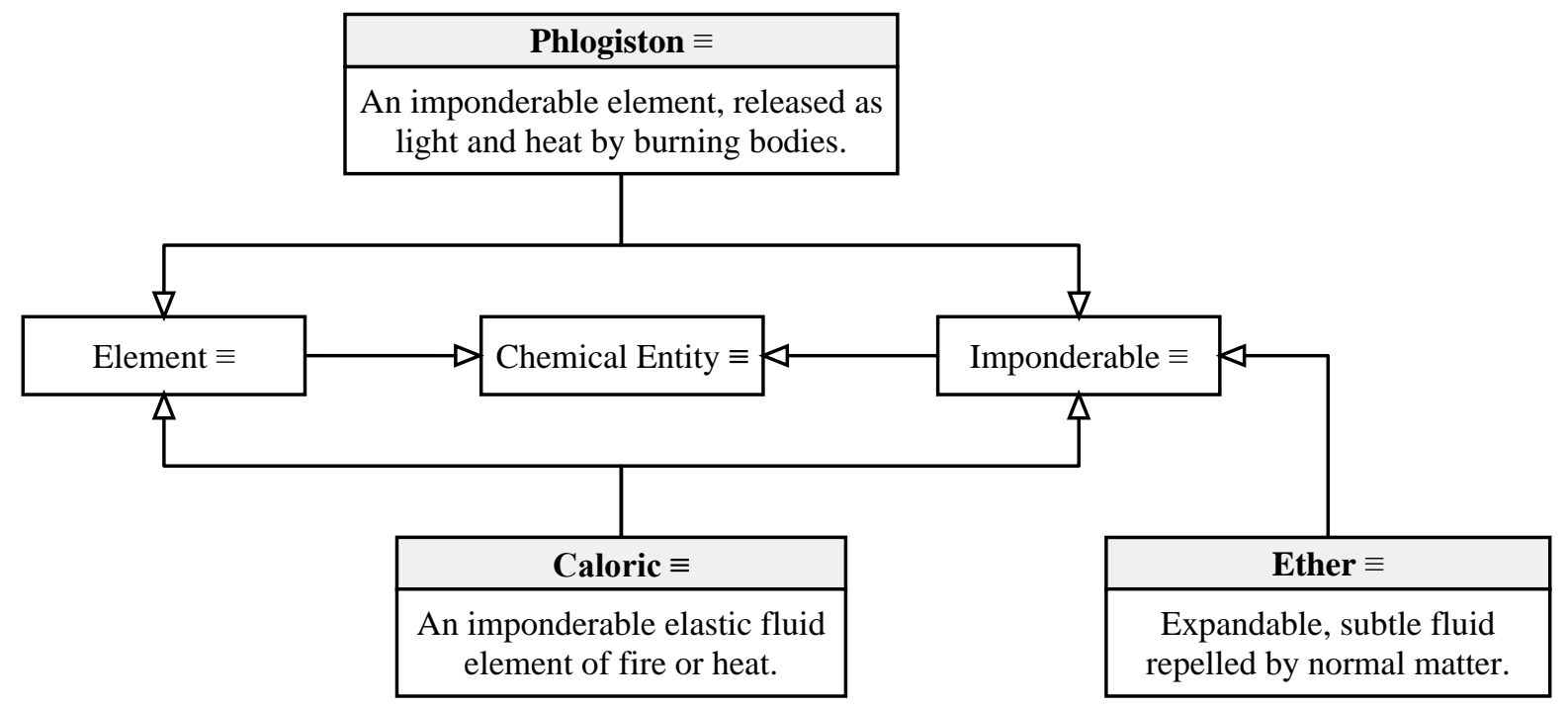

Note that since, in this diagram, some of the definitions are presented in the title-and-body mode, we had to ensure that all the definitions come with their respective triple bar symbols (三), for consistency.

Taxonomy diagrams can also visualize the relations of use between concepts, when one concept is used to define another concept. The following diagram depicts a fragment of the Newtonian taxonomy by showing the relations of use:

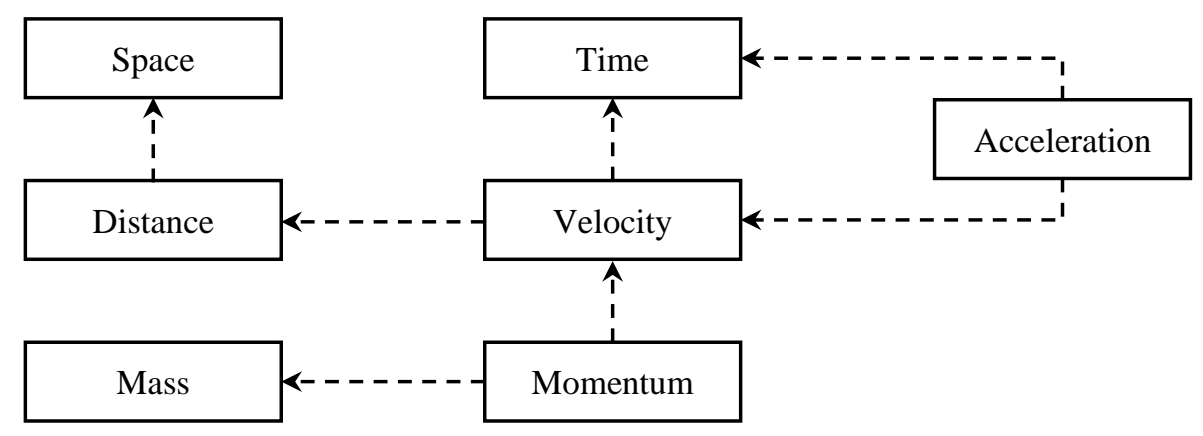

The diagram shows that acceleration is defined by means of the concepts of time and velocity, while velocity is defined by means of the concepts of time and distance, etc. Among other things, the diagram also highlights the more basic concepts of space, time, and mass, since the use arrows go only to them, not from them.

Naturally, taxonomy diagrams can be incorporated into larger diagrams, such as those depicting the key differences between taxonomies of two or more belief systems (see section Mosaic Comparison Diagram below). 


\section{Theory Relation Diagram}

Extracting the set of accepted theories and relations between them is often the single most fundamental task in the analysis of any belief system. Such extractions are best depicted in theory relation diagrams - arguably the most basic type of diagram. Diagrams of this type allow for the depiction of a variety of theory-theory relations, including implication, nonimplication, biconditional (equivalence), exclusive disjunction, consistency, inconsistency, compatibility, and incompatibility. The most common usage of theory relation diagrams is to show an agent's line of reasoning by means of implication arrows. The following diagram depicts reasoning related to the use of various surgical tools in medieval Islamic medicine (Zulfiqar \& Patton, forthcoming):

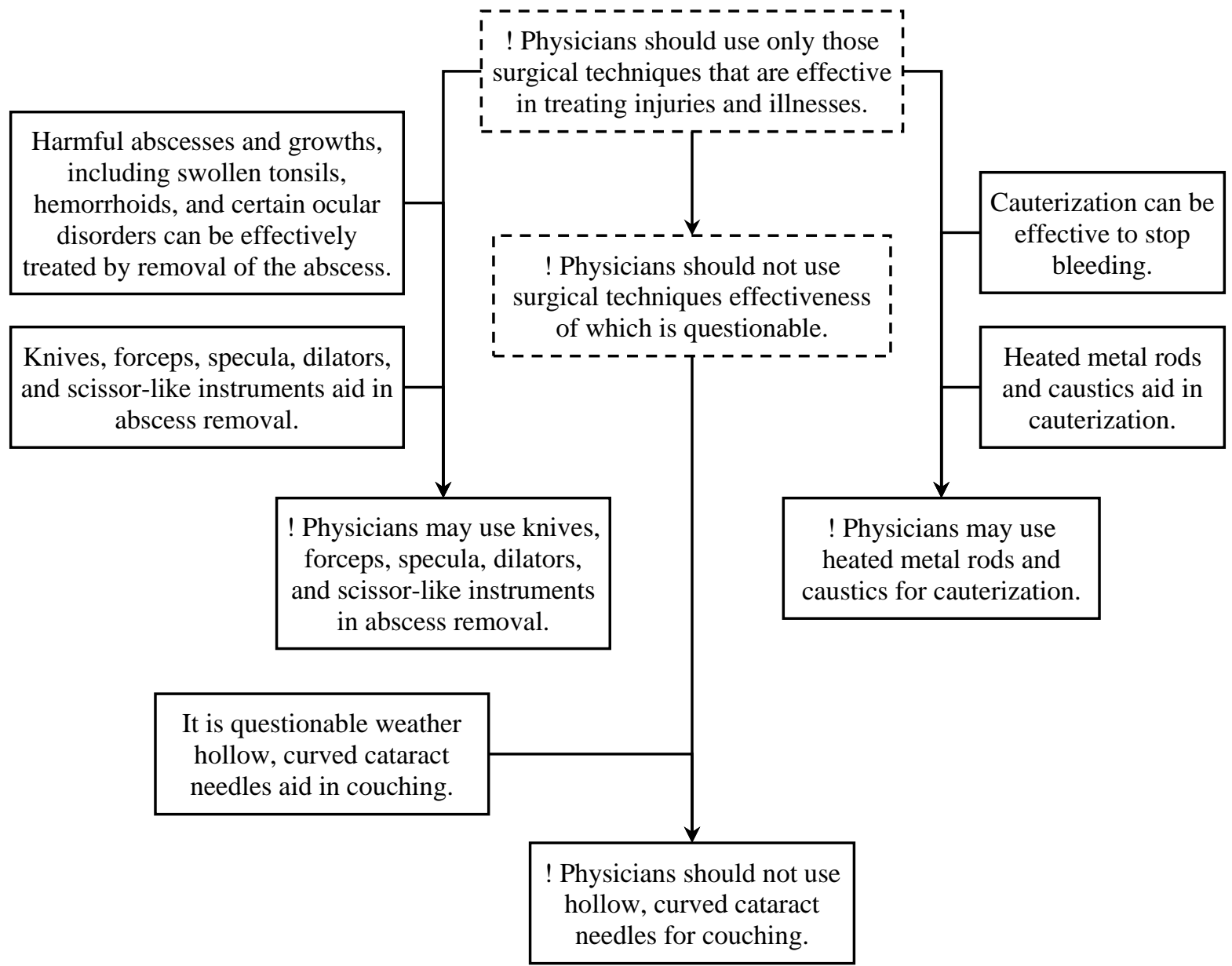

Among other things, diagrams of this type help identify tacit normative premises of arguments (depicted in a dashed rectangles).

A specific subtype of theory relation diagrams are diagrams visualizing relations between theories and methods. As theories and methods of their evaluation play central roles in any belief system, it is often important to highlight the relations between them. Specifically, it is often important to highlight which underlying theories a certain set of norms followed from. For example, the following diagram (adapted from Barseghyan, 2015, p. 149) shows how the requirements of the hypothetico-deductive method (HD method) follow deductively from the idea that the world as it appears in observations is a product of complex inner processes (i.e., there is more to the world than meets the eye) and the idea that any phenomenon can be given many different post-hoc explanations (i.e., it's easy to be smart after the fact): 


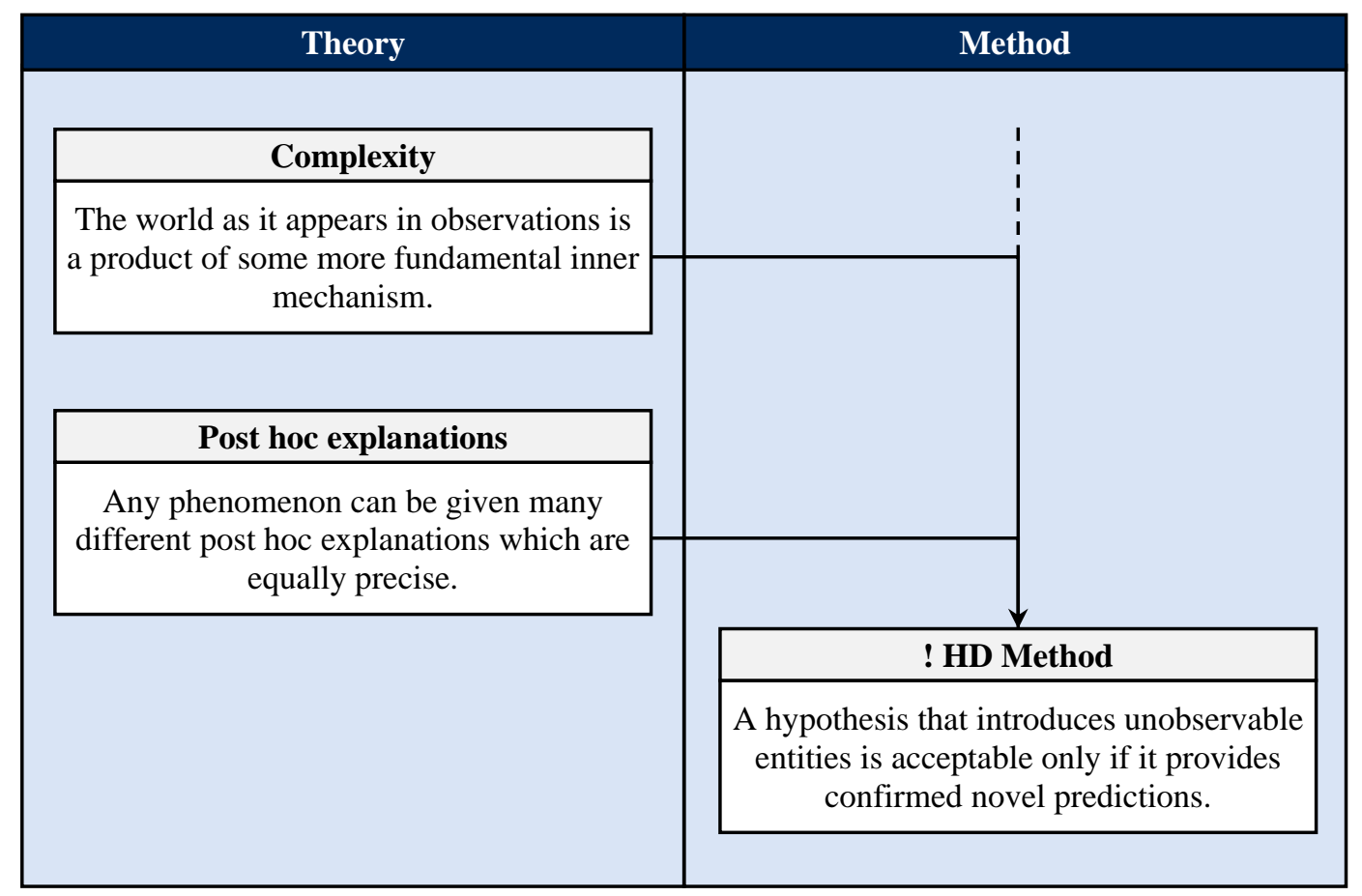

Note the dashed line indicating that we have skipped some of the other premises. Here is a similar diagram from the scientonomic literature (adapted from Fatigati, 2017, p. 26) showing the deduction of the contemporary method for identifying the accepted theories in the Medieval Arabic Scientific Mosaic (MASM):

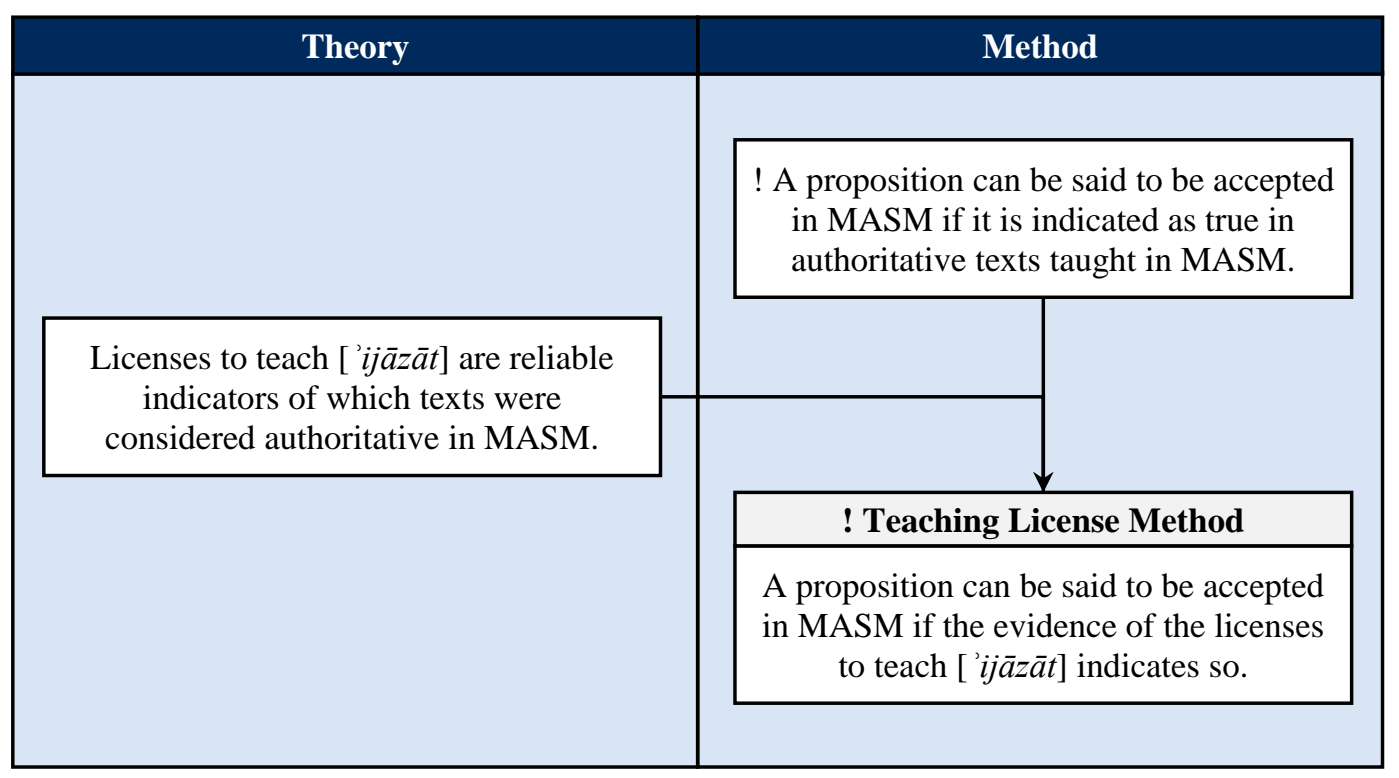

Such diagrams can also be handy when depicting the relations of satisfaction between a theory and the method which it satisfied: 


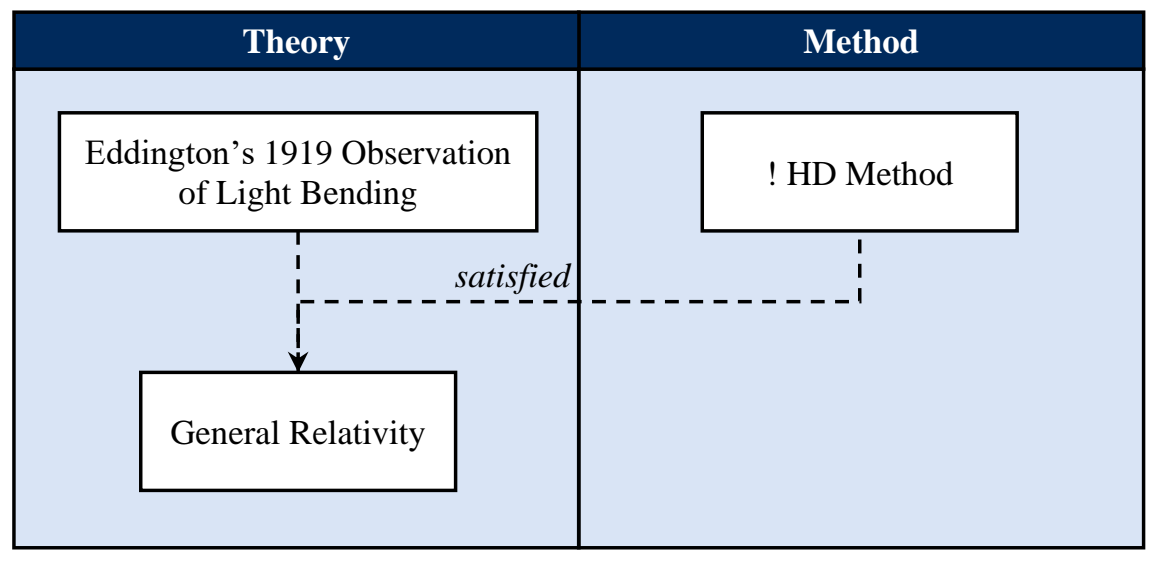

In addition, theory relation diagrams can also depict the relation of implementation, where a more specific method implements the requirements of a more abstract method. Here is an example of such a diagram (adapted from Barseghyan, 2015, p. 152):

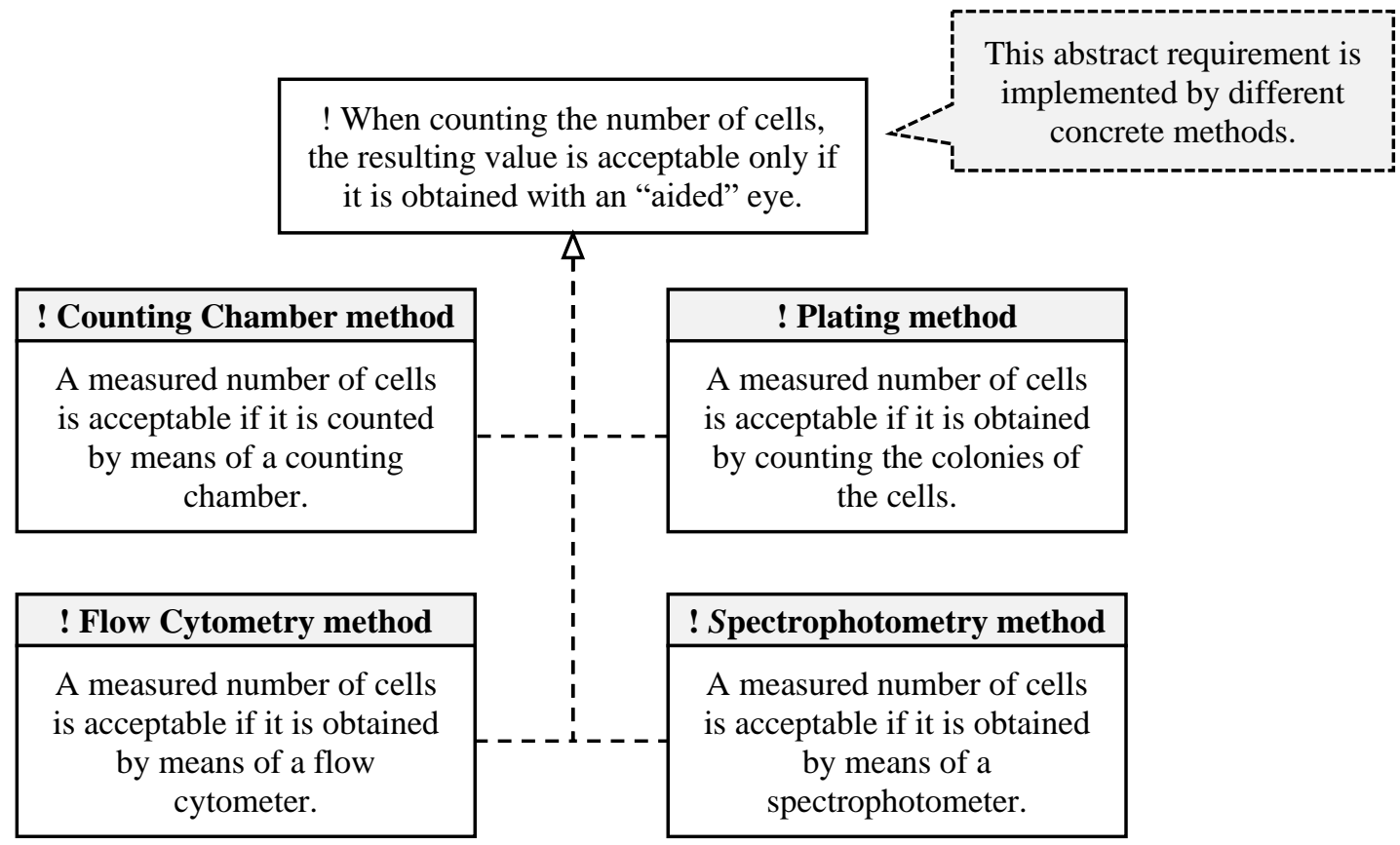

In principle, with the use of (labelled) arrows, theory relation diagrams can depict any of the relations that obtain between theories.

\section{Question-Theory Diagram}

A systematic study of a belief system implies a detailed analysis of the respective epistemic agent's questions and answers (i.e., theories). As questions can presuppose any number of theories and as theories are answers to questions, question-theory diagrams are particularly useful for visualizing the intricate tapestry of relations between different theories and questions. While question-theory diagrams are a useful visualization tool in any media, they are of particular use in electronic media that do not impose strict spatial limitations. Such diagrams can include a great number of elements and can be very useful for navigating through the agent's belief system. The following question-theory diagram depicts a fragment of Medieval and early modern cosmological beliefs, from the question about the distance between the Earth and the sphere of the stars and all the way up to the respective definitional questions (adapted from Barseghyan \& Levesley, 2021, p. 9): 


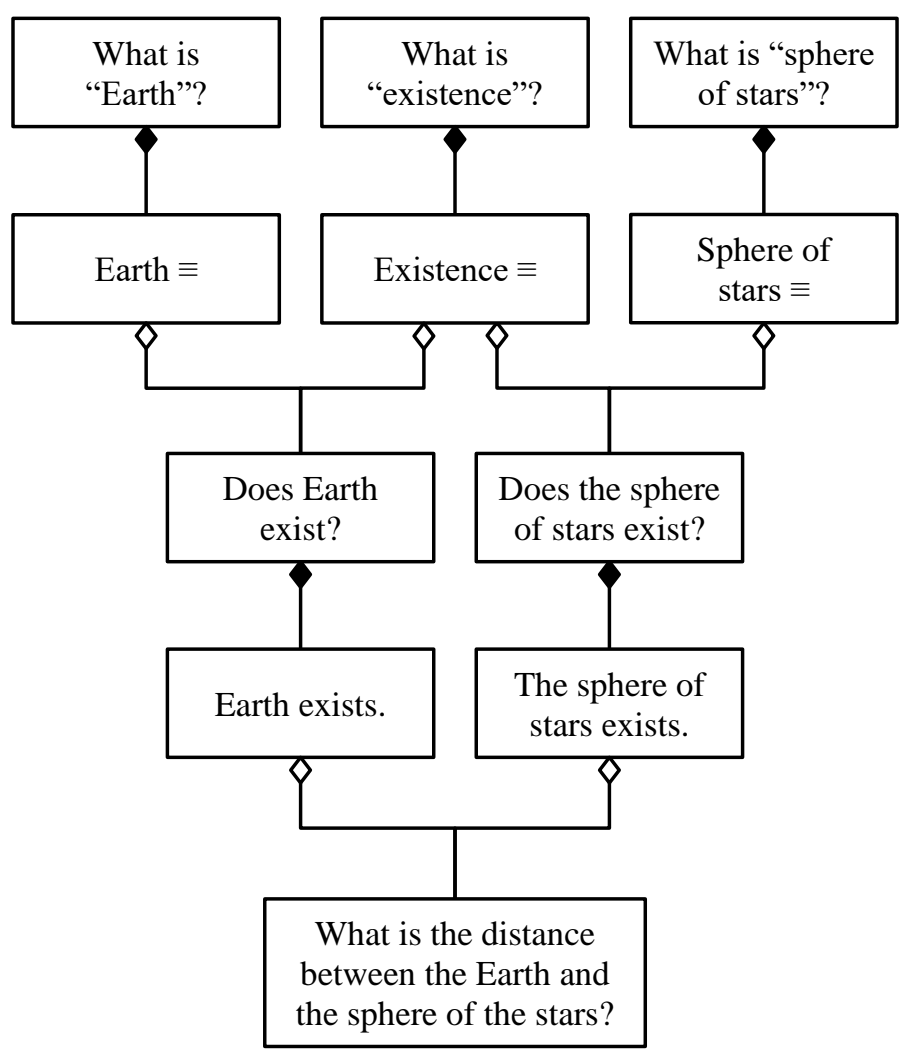

As discussed in section Question-Theory Relations, the standard UML symbol for aggregation (white diamond) indicates that a question presupposes a theory, while the standard UML symbol for composition (black diamond) indicates that a theory is an answer to a question.

\section{Mosaic Comparison Diagram}

The primary task of mosaic comparison diagrams is to show the overlap and differences between two or more belief systems. Here is a diagram comparing three belief systems concerning the question of the immortality of the soul:

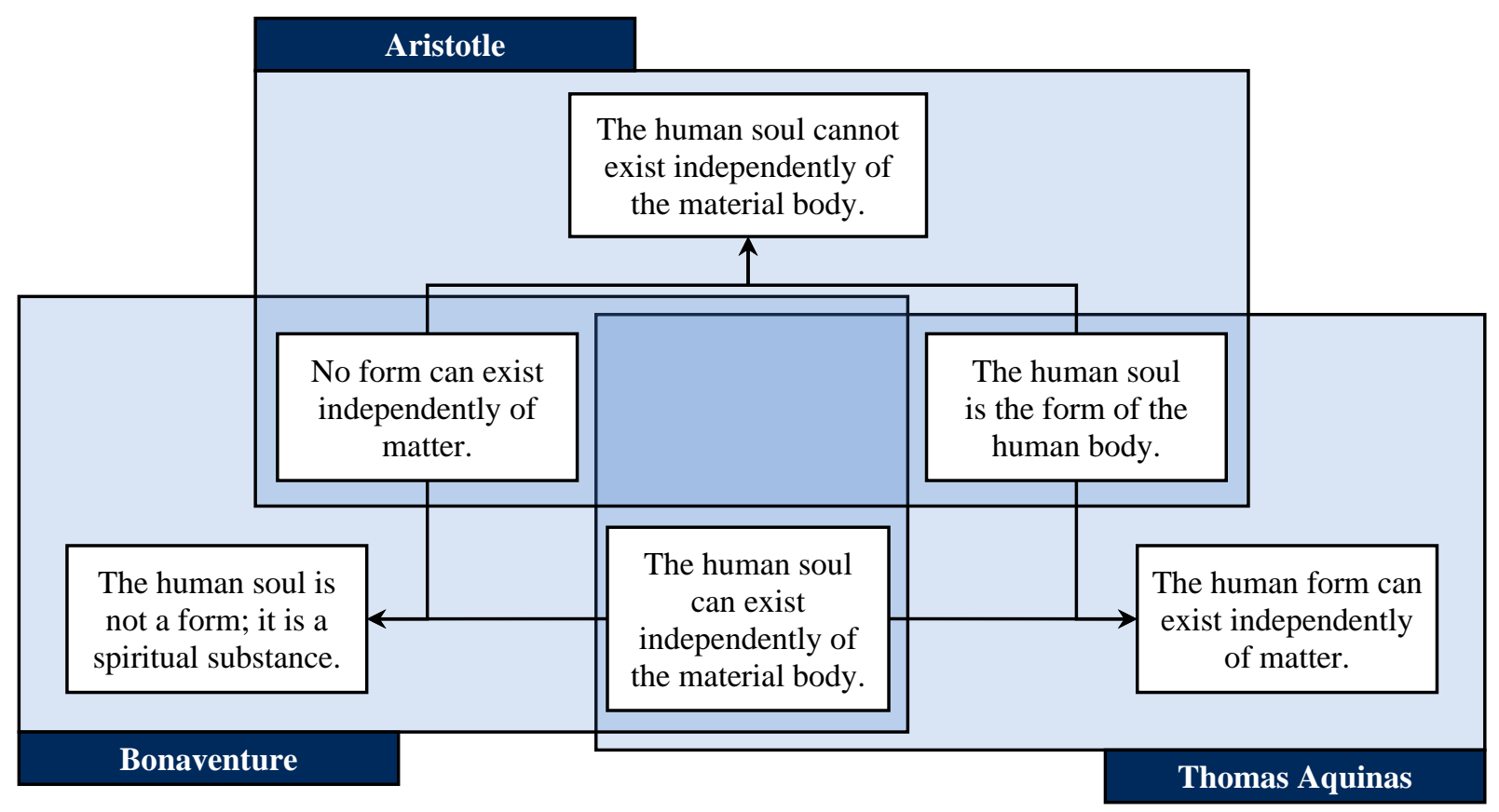


The diagram illustrates both the overlap and differences between the three positions and highlights the respective lines of reasoning.

Mosaic comparison diagrams are essentially elaborate versions of Venn diagrams (Venn, 1880), where overlapping regions indicate shared epistemic elements. One major advantage of depicting mosaics as rectangles rather than circles is that rectangles are much better suited for housing the rectangular boxes of epistemic elements, such as theories, question, and methods. Anyone who has attempted to position rectangular text boxes into circles of a Venn diagram will certainly appreciate this.

\section{Agent (Mosaic) Relation Diagram}

Diagrams of this type illustrate synchronic relations between different agents or mosaics, such as the relations of authority delegation or the relation of super-community/sub-community. We have already presented a variety of different diagrams depicting the relation of authority delegation (see section Epistemic Collections). The following diagram depicts yet another case of authority delegation (Loiselle, 2017):

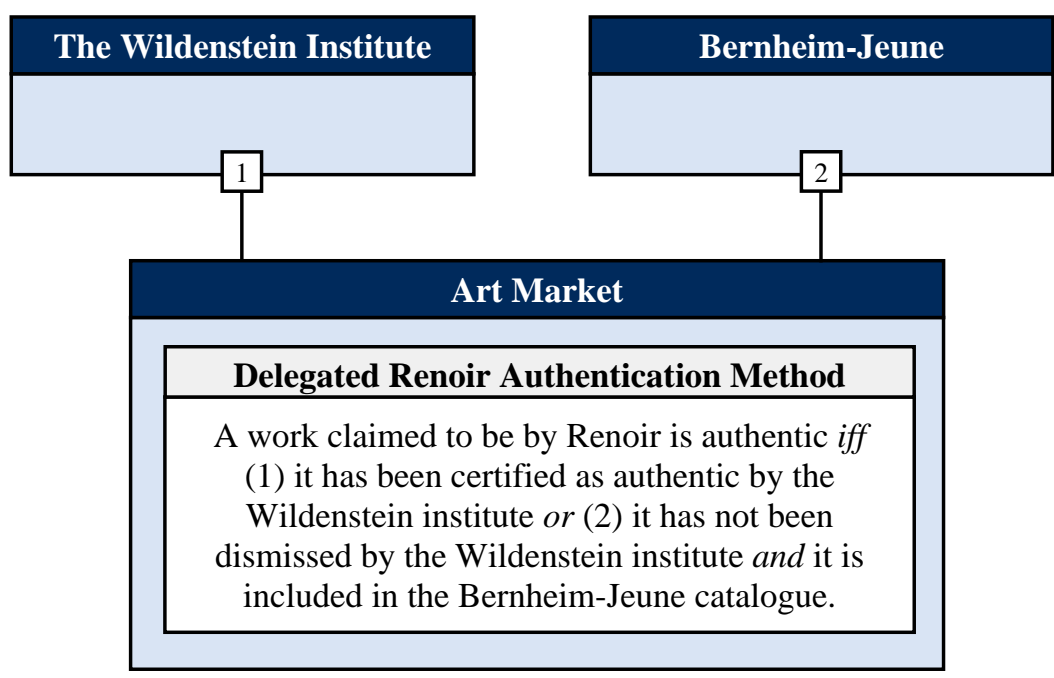

Note that the numbers within the authority delegation's boxed-connectors indicate the hierarchy of delegation: for the art market community, the Wildenstein Institute is the first expert on Renoir, while Bernheim-Jeune is consulted only when the Wildenstein Institute has not expressed its position on the respective painting. Naturally, the same relationship can be depicted by replacing the mosaic folders with agent stick-figures:

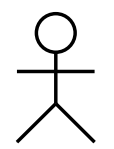

The Wildenstein Institute

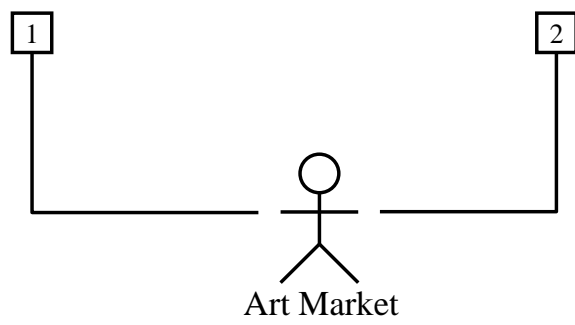

Diagrams of this type may also be useful for visualizing the structure of agents' membership in different communities. The diagram below visualizes how Aryan physicist viewed the membership of various subcommunities of physicists in the broader community of German physicists (adapted from Rapson \& Shaw, forthcoming): 


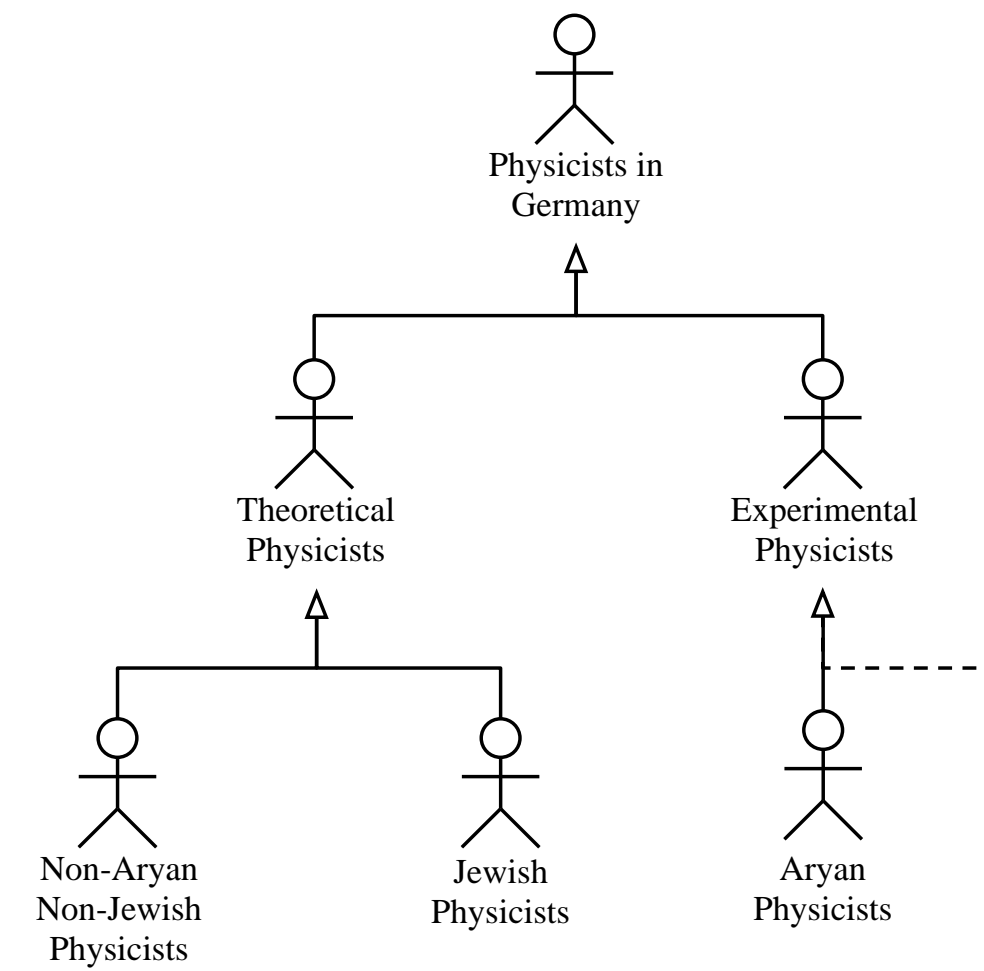

In principle, it is possible to depict community-subcommunity relations and authority delegation relations in the same diagram. However, to avoid unnecessary clutter, we recommend staying away from combining the two relations in the same diagram. While there are no conceptual counterindications here, such hybrid diagrams simply do not look good (which is not to say that they should be avoided at all costs).

\section{Diachronic Diagrams}

The purpose of diachronic diagrams is to depict processes, i.e., temporal sequences of events. With our diagrammatic notation at hand, we can now construct a variety of diachronic diagram types for depicting epistemic evolution across time. Generally, timelines are an obvious choice for visualizing the temporal evolution of any system; belief systems are no exception from this rule. Timelines can be very useful when displaying the evolution of agents' stances toward different epistemic elements over time as well as showing causal influences between agents. Here we will consider event timelines, element-stance timelines, agent-question-theory timelines, splitmerge timelines, and influence diagrams.

\section{Event Timeline}

The most basic type of diachronic diagram is the event timeline, the main purpose of which is to succinctly visualize a sequence of events. Event timelines are not specific to belief systems, since any temporal sequence can be portrayed using an event timeline. So, our task here is to focus on those aspects of event timelines that are of particular import when dealing with the histories of belief systems. Specifically, event timelines can include not only events with precise historical data, but also events for which precise historical data is lacking, i.e., events which we have been able to narrow down to an ambiguous time range. In the latter case, the imprecision of the historical data can be shown by means of range lines, as explained in the section Imprecise or Missing Data, above. Event timelines can also include range bars to depict events (or series of events) that took place over an extended period. Here is an event timeline with two period bars, a range line that indicates lack of historical precision, and two events whose precise dates are known (adapted from Levesley \& Barseghyan, forthcoming): 


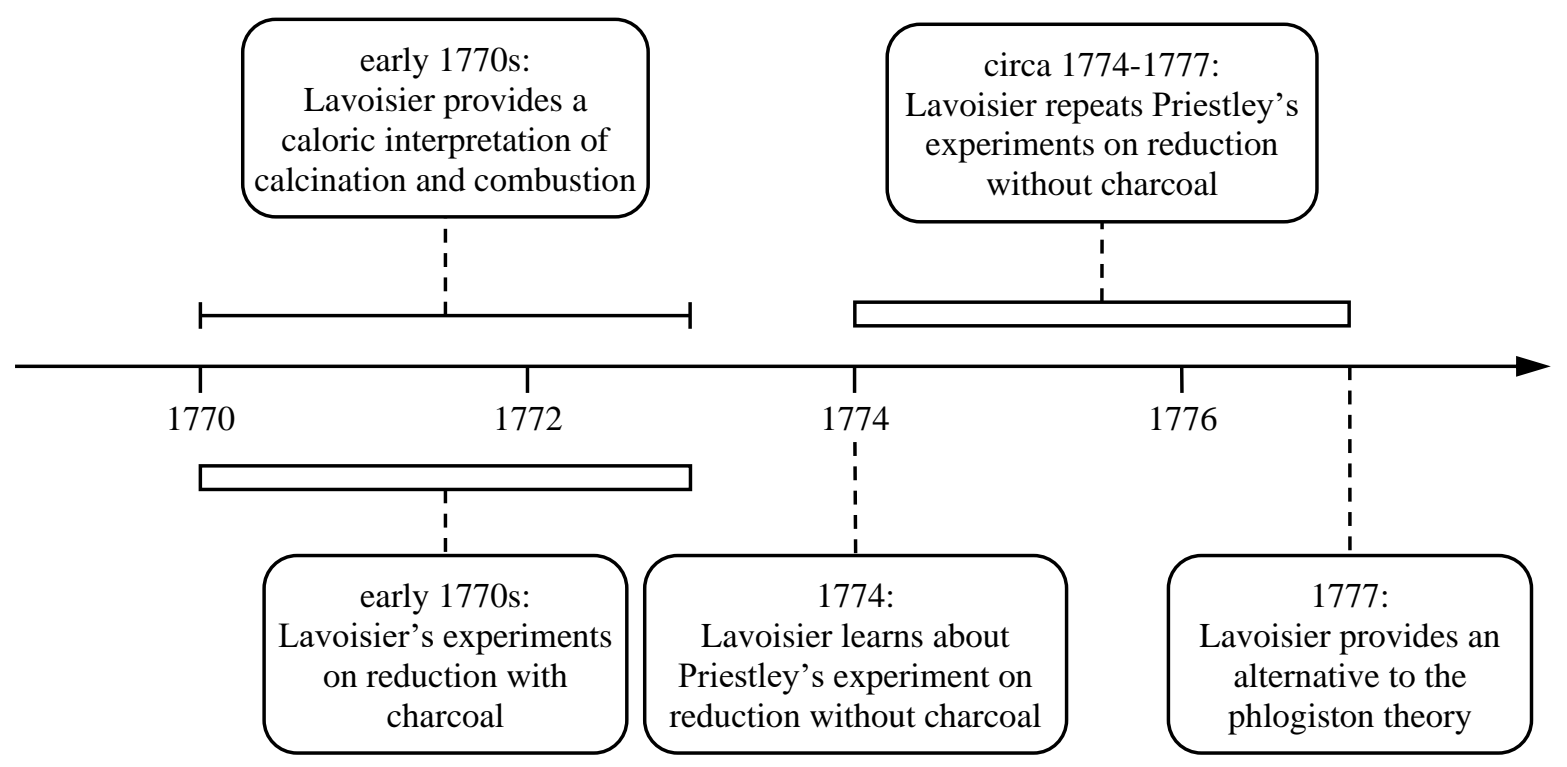

\section{Element-Stance Timeline}

The purpose of element-stance timelines is to visualize the evolution of different stances of one agent towards one or more epistemic element in the same diagram. Here is an example depicting the evolution of stances of the $19^{\text {th }}$ century brain research community towards cortical (non-)localization (adapted from Sarwar \& Patton, forthcoming):

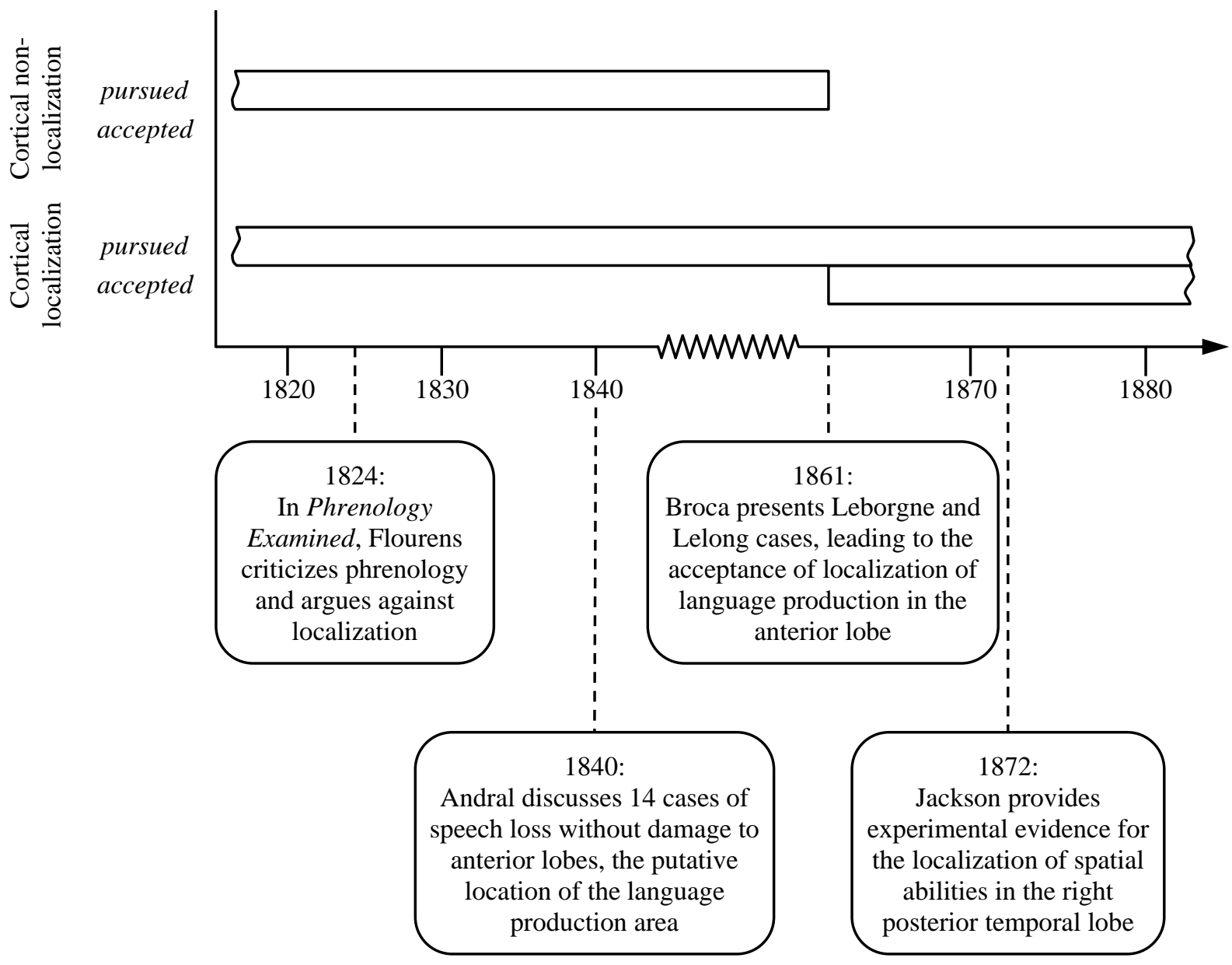


The potential advantages of element-stance timelines are clear. For one, they show the co-evolution of an agent's stances towards several epistemic elements. They also highlight any missing or imprecise historical data and thus indicate potential areas for historical research. Moreover, one may additionally create a sort of hybrid diagram, as depicted above, where notable events are highlighted underneath the timeline.

\section{Agent-Question-Theory Timeline}

Timelines of this kind can be used to illustrate an evolution of beliefs of one or many agents on one or many questions. First, a timeline might be used to display the evolution of a mosaic of a single epistemic agent. In such a case, each horizontal line can represent the history of the agent's answers to one specific question:

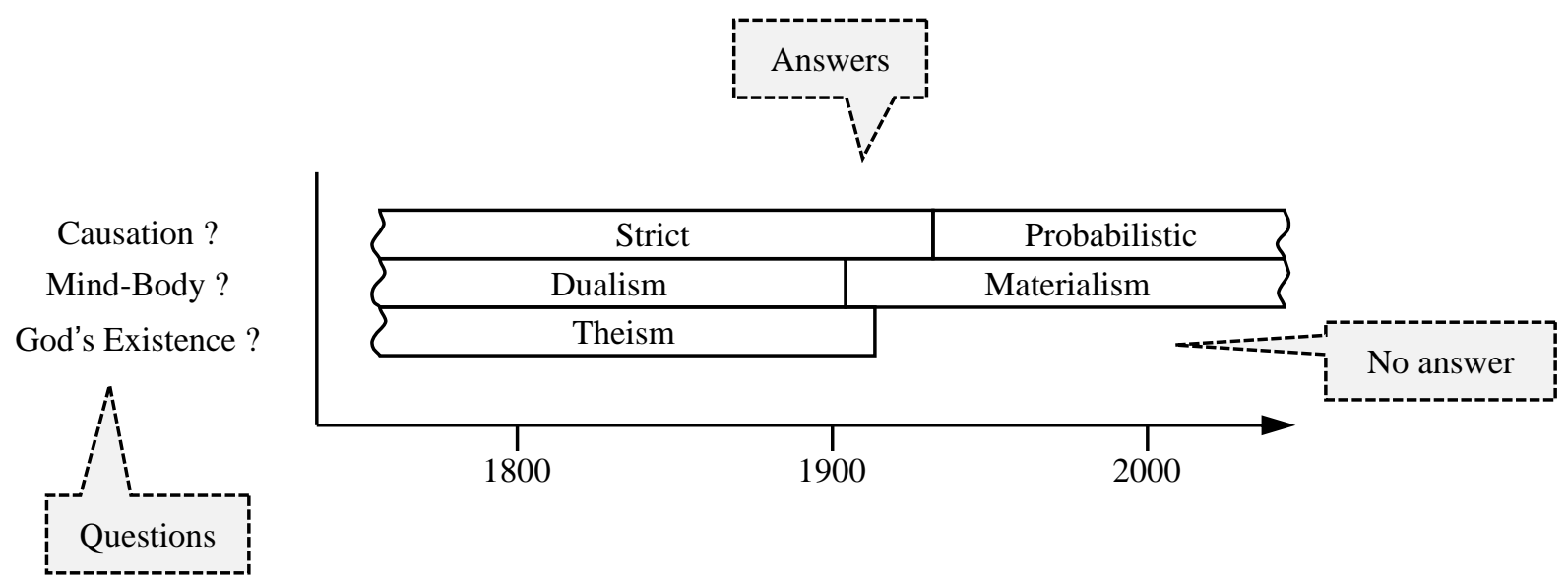

Diagrams of this type are useful when it is important to display the co-evolution of an agent's accepted answers to different questions.

Another variation of this diagram type is a timeline displaying the co-evolution of the accepted answers of several agents to a select question. In such a case, the question itself is omitted from the diagram and the evolution of each agent's answers to the question is represented by one horizontal line. The following timeline illustrates the co-evolution of accepted natural philosophies for three different communal agents:

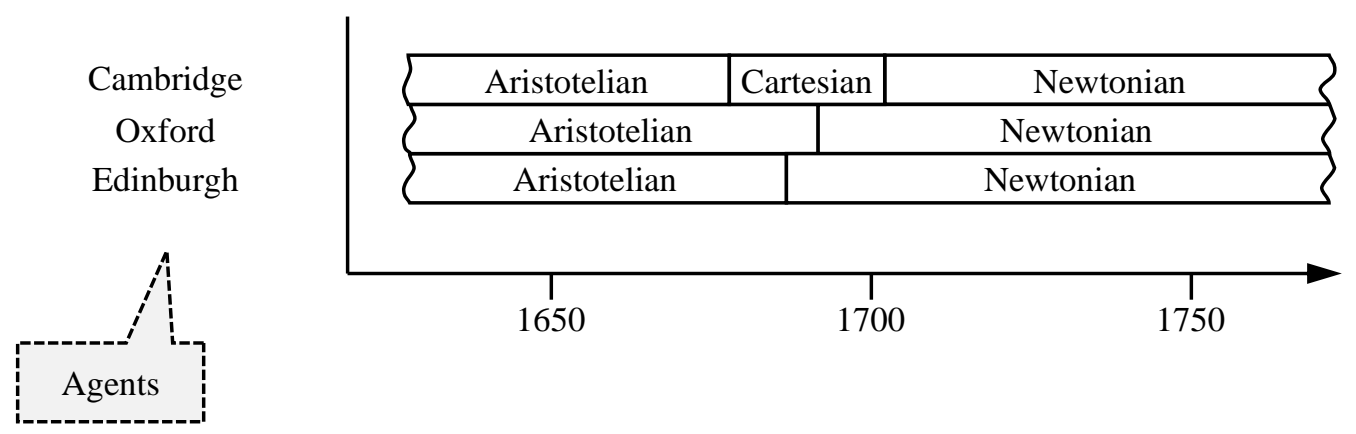

Thus, a timeline can display the accepted answers of a single agent to many questions, or those of many agents to one question.

It is also often necessary to portray the co-evolution of the accepted answers of several agents to several questions. Here is an example comparing the accepted answers of the French chemical community, Lavoisier, and Priestley to three central questions concerning the existence of phlogiston and caloric as well as the entities involved in reduction reactions (adapted from Levesley \& Barseghyan, forthcoming): 


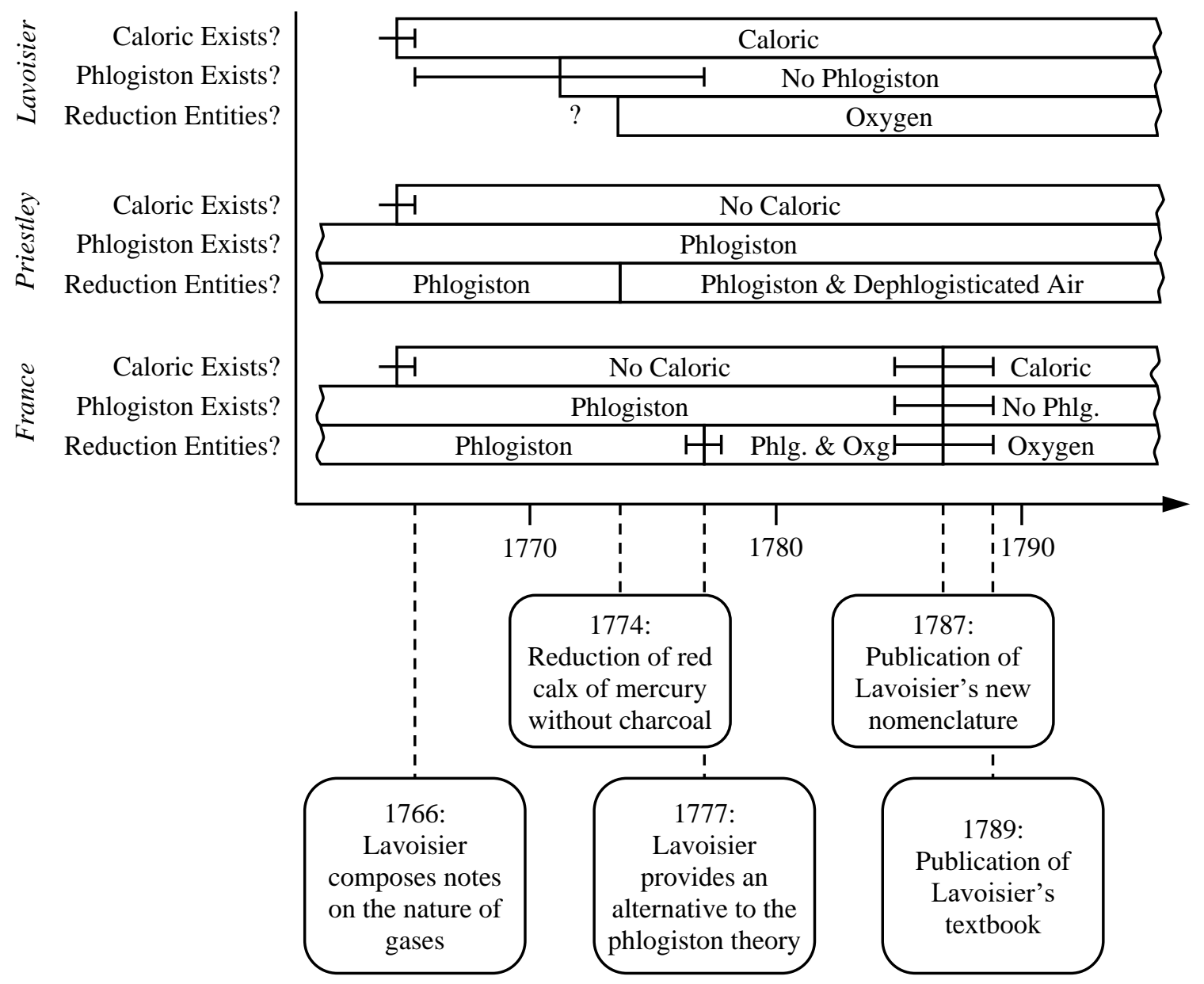

For consistency, we suggest reserving the horizontal axis for time in all timelines. In addition, we suggest using stacked/clustered bars to easily compare the stances of the various agents on the various questions. It is permissible to use highlighting to sharpen the difference between different theories (see section Highlighting and Callouts below). Depending on the ratio of questions and agents, as well as the specific needs of the visualization, it might be necessary to stack the answers of different agents on the same question together to highlight the differences and similarities between them as is done above. Furthermore, it is possible to hybridize the diagram by adding events on the same timeline, as depicted above.

\section{Split-Merge Timeline}

In addition, communal epistemic agents can split and merge. One way of portraying mosaic splits and merges is by using agent-question-theory timelines, like the one below:

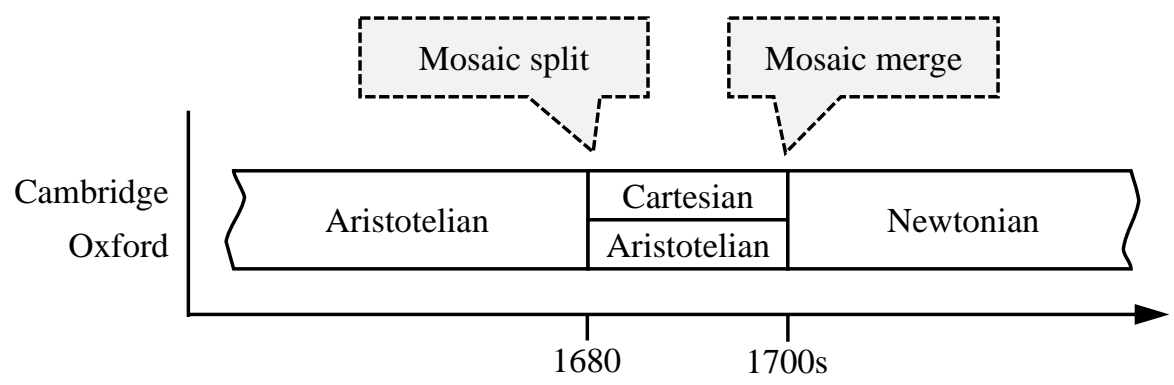

Note that, in this timeline, the respective question is omitted. 
Naturally, such diagrams assume that there is a way of identifying the splitting and merging of communities by some persistent identifier, e.g., geographic, national, institutional, disciplinary, etc. If the respective theories can be omitted, the same process can be portrayed by using agent symbols and forking influence arrows:

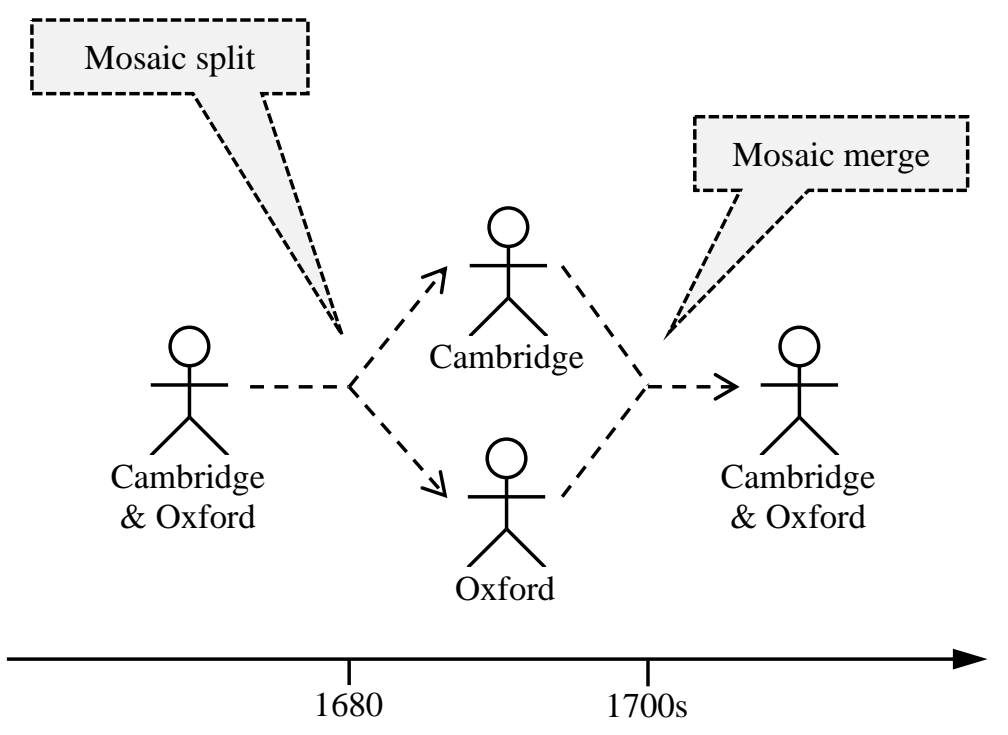

To indicate that a mosaic split was necessary rather than possible, solid line arrows are to be used (see section Influences for details):

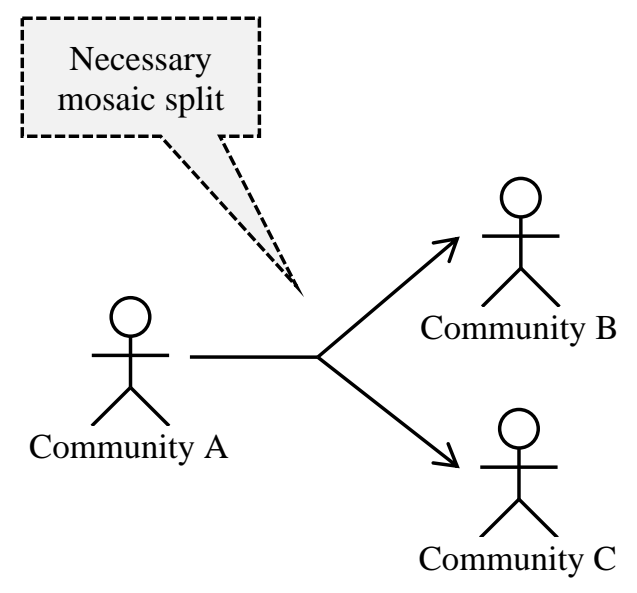

\section{Influence Diagram}

Agents and events can influence other agents and events. Depicting such influences is the primary function of influence diagrams. As influences are, by definition, temporal in nature, the influence relations are to be depicted by means of temporal influence arrows (see section Influences). For instance, in the following diagram, generic influence arrows are used to portray influences between diverse epistemic agents: 


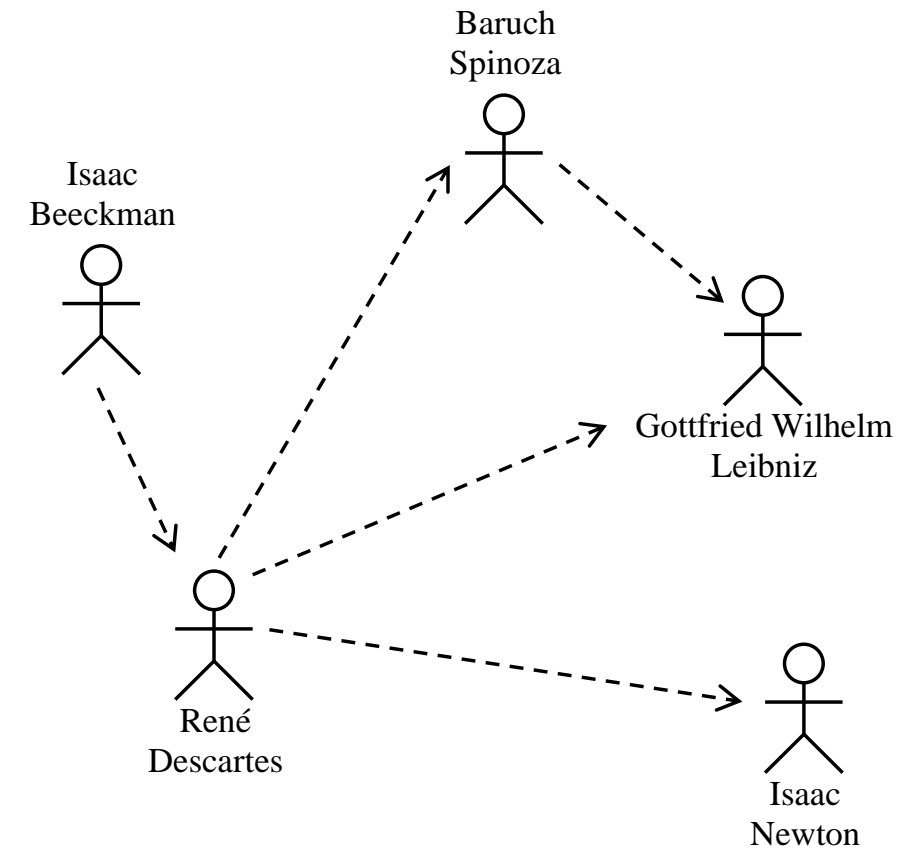

Influence diagrams may also be combined with a timeline, if necessary. Here is the same agent-influence diagram positioned on a timeline, that also indicates the lifespans of the agents with horizontal bars:

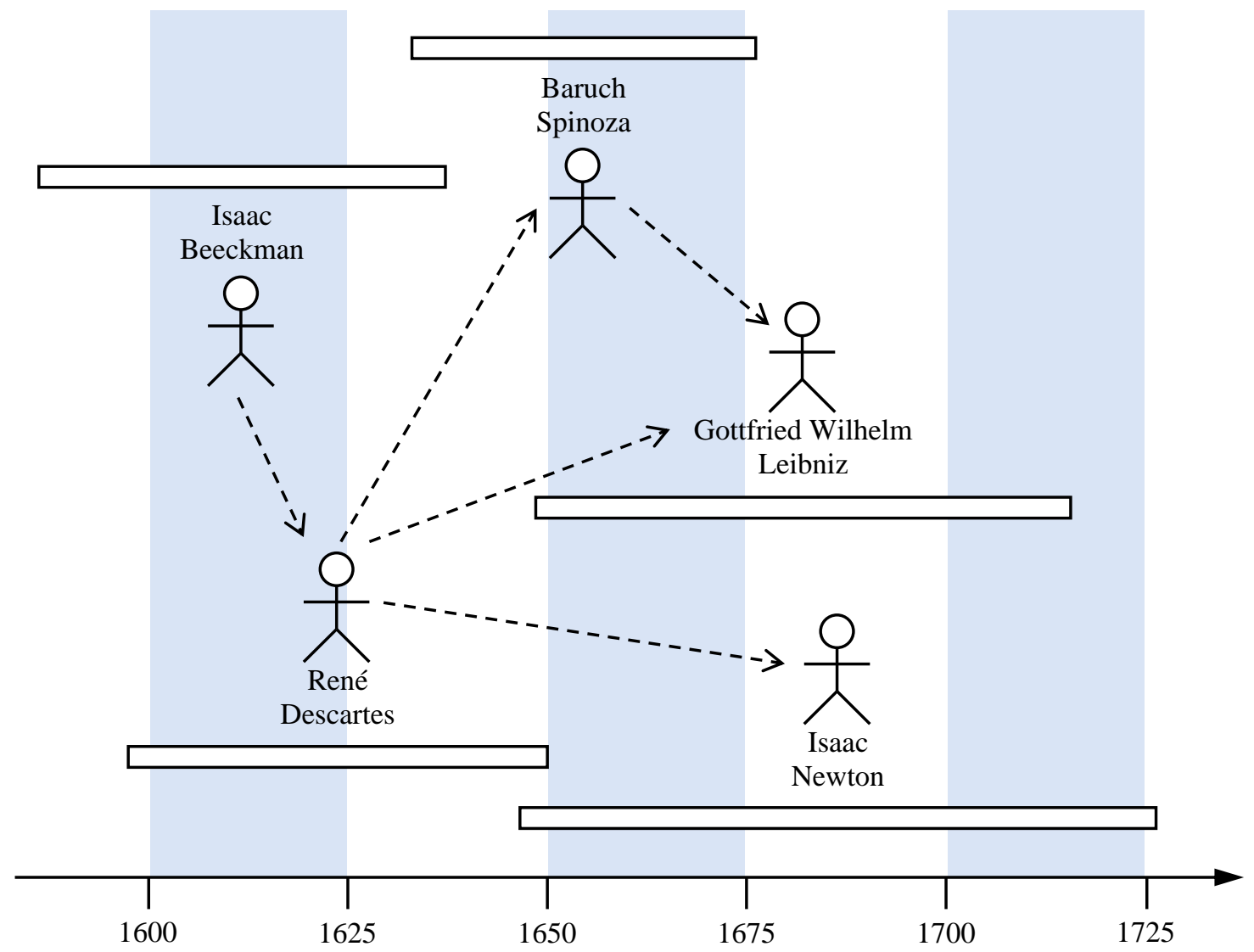

Also note the optional highlighting.

Influence diagrams are also ideal for showing how some events led to other events. The following diagram illustrates a complex tapestry of the major developments in the late- $18^{\text {th }}$ century chemistry (adapted from Levesley \& Barseghyan, forthcoming): 


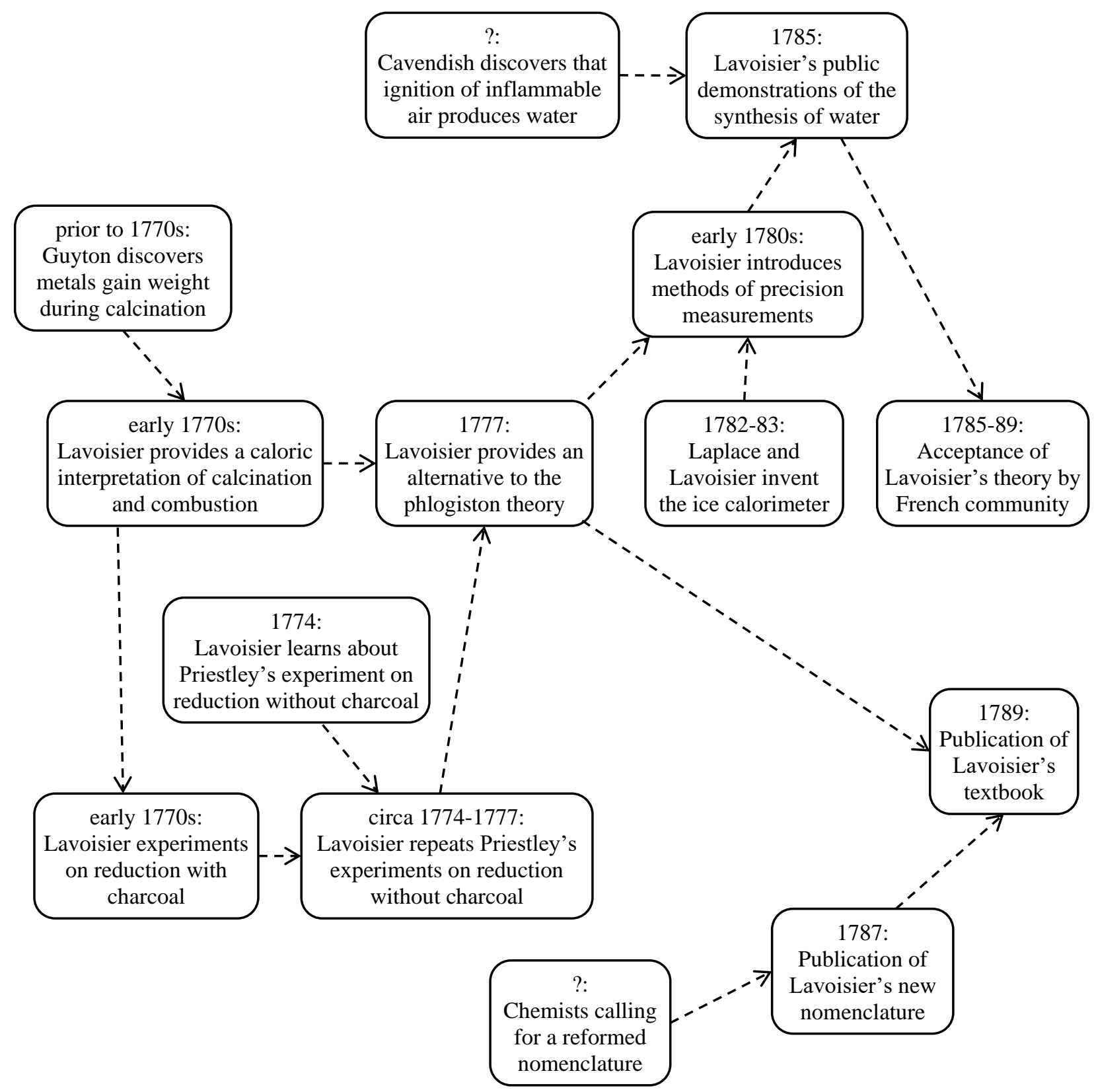

Such influence diagrams will be of particular use to historians trying to make sense of complex historical developments. They can help historians to identify missing links between different events and highlight areas for future research.

\section{Areas for Further Research}

We would like to conclude this paper by identifying several areas for future elaboration of this notation.

\section{Compound Elements}

The nature of compound elements is not well understood in scientonomy. For instance, it is not quite clear what it means scientonomically to say that Newton's second law was part of Newton's mechanics. Similarly, the relation between a method and its constituent criteria has not yet been properly conceptualized (Mercuri \& Barseghyan, 2019). Thus, we are not yet in a position to suggest visualization tools for depicting that a certain element was part of a compound element. 


\section{Epistemic Practice}

As any visualization presupposes a solid theoretical foundation, and as no such foundation exists for epistemic practice, we do not offer any means of visualizing the activities of day-to-day knowledge production processes. The ontology of epistemic practice is one obvious area of future research in scientonomy (Allen \& Barseghyan, forthcoming). Once we have a clear picture of entities, relations, and actions involved in epistemic practice, we can then think of ways to visualize them.

\section{Individuals and Communities}

More work needs to be done in theoretical scientonomy on the relations between communal and individual agents. When the nature of these relations becomes clearer, we might need to develop diagrammatic tools to visualize these relations. At this stage it is unclear if it is scientonomically important to depict a community's network.

\section{Non-deductive Implication Arrows}

Solid line connectors with stealth/notched arrowheads are currently used generically in theory relation diagrams to depict logical implications. This leaves unresolved the issue of whether it is important to symbolically distinguish classical logic deductions from other forms of inference, such as inductive inference. This is seen, for instance, in the following diagram where empirical evidence is used in support of general hypotheses (adapted from Gilboa, Slawnych, \& Palider, forthcoming):

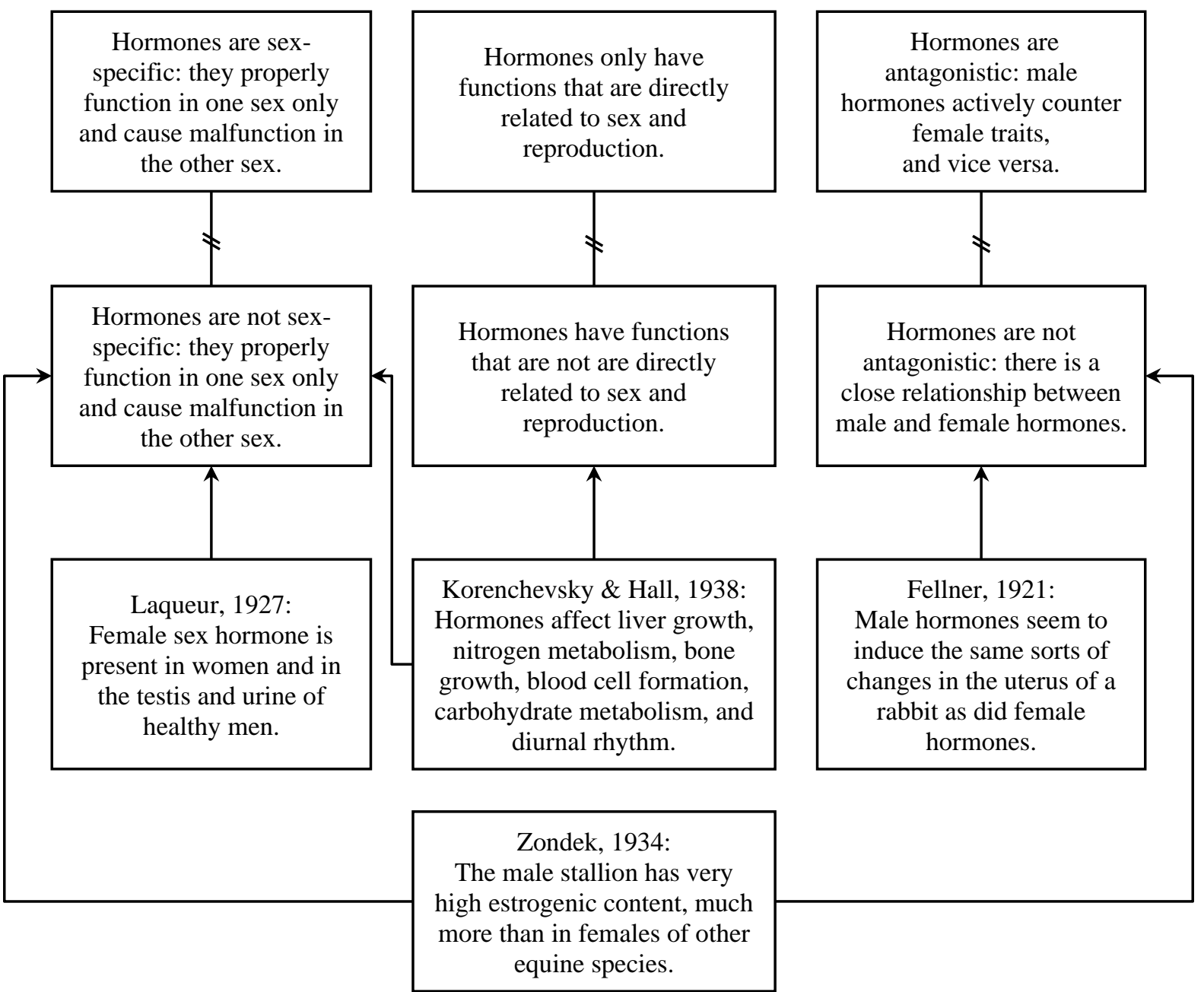

This kind of problem exists for all forms of non-deductive inference. One potential solution is to label all of the arrows as inductive, however this needs to be discussed further by the community. It may be the case, for instance, 
that all inductive inferences are better diagrammed with a normative principle of induction as a form of theorymethod diagram.

Furthermore, although no diagramming efforts have called for it yet, there may be instances where different agents employ different rules of inference in their reasoning. This could make the agents qualify the other's reasoning as non-deductive, while claiming their own reasoning as deductive. With the current diagrammatic notation, there is no simple way of depicting this (other than by labeling the respective implication arrows or using callouts). The question of what an arrow ought to depict may also be raised. Are they meant to depict any instances of logical implication relation between different elements (including those of which the respective historical agent was not aware), or only the agent's own reasons for accepting the conclusion (see Palider, 2019)? As reasons are agent-relative, and as agents need not necessarily be aware of or accept all of the logical implication relations among their epistemic elements, the two approaches may produce very different depictions. A diagram visualizing the agent's reasons may be very different from a diagram that visualizes implication relations in the agent's mosaic. More research is needed here.

\section{Epistemic Tools}

Epistemic agents are typically aided in their work of creating, disseminating, and preserving knowledge by a plethora of physical objects and systems - such things as a ruler, quill pen, book, astrolabe, particle accelerator, robotic Mars rover, or the Internet. To encompass the role of such tools and instruments in scientonomy, Patton (2019) has proposed the concepts of epistemic tool:

\begin{tabular}{|c|}
\hline Epistemic Tool $\equiv$ \\
\hline A physical object or system is an epistemic tool \\
for an epistemic agent iff there is a procedure by \\
which the tool can provide an acceptable source \\
of knowledge for answering some question under \\
the employed method of that agent. \\
\hline
\end{tabular}

The relationship between an epistemic agent and a tool is thus a consequence of that agent's employed method. In the proposed addition to the scientonomic ontology, this relationship is understood as one of tool reliance:

\section{Tool Reliance $\equiv$}

An epistemic agent is said to rely on an epistemic tool iff there is a procedure through which the tool can provide an acceptable source of knowledge for answering some question under the employed method of that agent.

The relationships between communal and individual epistemic agents and their tools are central to the creation of knowledge and require diagrammatic depiction. The following diagram uses the symbols suggested by Patton to depict an agent, an epistemic tool, and the relation of tool reliance between them:

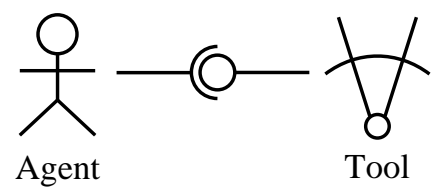

To illustrate the usefulness of these concepts and diagrammatic symbols, Patton (2019) analyzed the American National Aeronautics and Space Administration's (NASA) Hubble Space Telescope (HST) project, identifying the epistemic agents and epistemic tools involved, and their relationships of authority delegation and tool reliance. The resulting diagram illustrates the proposed usage of symbols to depict epistemic tools and the relationship of 
tool reliance. The diagram depicts the relationships between the $H_{0}$ key group, a team of 15 astronomers tasked with determining the Hubble constant $H_{0}$ (a parameter that is a measure of the rate of the expansion of the universe) and several communities and epistemic tools within NASA.

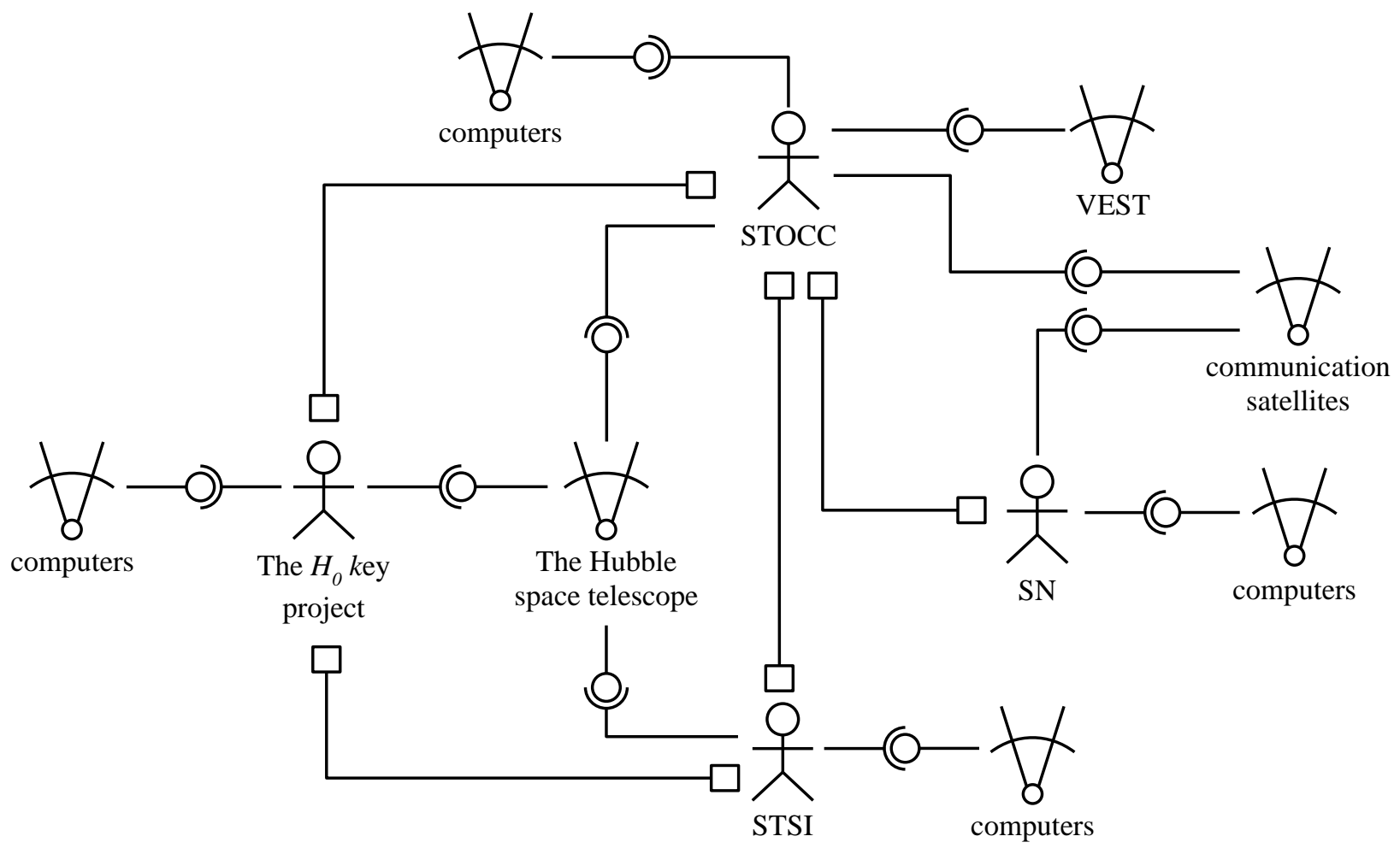

These proposed new additions to the scientonomic ontology and their diagrammatic representations are still being reviewed for acceptance by scientonomy community as of this writing.

\section{Suggested Modifications}

As the paper presents a notation de facto accepted by the community, no new suggested modifications are required here. The respective pages of the encyclopedia will be updated upon the publication of this paper to ensure that the conventions of the diagrammatic notation are properly documented.

\section{References}

Allen, J. \& Barseghyan H. (forthcoming). Scientific Practice in the Scientonomic Framework. Scientonomy.

Barseghyan, H. (2015). The Laws of Scientific Change. Springer.

Barseghyan, H. (2018). Redrafting the Ontology of Scientific Change. Scientonomy 2, pp. 13-38. Retrieved from https://scientojournal.com/index.php/scientonomy/article/view/31032.

Barseghyan, H. (2021). Diagramming Imprecise and Incomplete Temporal Information. In Basu et al. (Eds.) (2021).

Barseghyan, H. \& Levesley, N. (2021). Question Dynamics. Scientonomy 4, pp. 1-19. Retrieved from https://scientojournal.com/index.php/scientonomy/article/view/37120.

Barseghyan, H.; Patton, P.; \& Shaw, J. (Eds.) (forthcoming). Visualizing Worldviews: A Diagrammatic Notation for Belief Systems.

Basu, A.; Stapleton, G.; Linker, S.; Legg, C.; Manalo, E.; \& Viana, P. (Eds.) (2021). Diagrammatic Representation and Inference. 12th International Conference, Diagrams 2021, Virtual, September 28-30, 2021, Proceedings. Springer.

Cumming, G.; Fidler, F.; \& Vaux, D.L. (2007). Error Bars in Experimental Biology. The Journal of Cell Biology 177(1), pp. 7-11.

Fatigati, M. (2017). A Method for Reconstructing the Medieval Arabic Scientific Mosaic. Scientonomy 1, pp. 19-28.

Retrieved from https://www.scientojournal.com/index.php/scientonomy/article/view/27761. 
Fine, A. (2017). The Einstein-Podolsky-Rosen Argument in Quantum Theory. In Zalta, E. N. (Ed.) (2017). The Stanford Encyclopedia of Philosophy (Winter 2017 Edition). Retrieved from https://plato.stanford.edu/archives/win2017/entries/qt-epr/.

Fraser, P. \& Sarwar, A. (2018). A Compatibility Law and the Classification of Theory Change. Scientonomy 2, 67-82. Retrieved from https://scientojournal.com/index.php/scientonomy/article/view/31278.

Gabbay, D.; Horty. J; Parent, X.; van der Meyden, R.; \& van der Torre, L. (Eds.) (2013). Handbook of Deontic Logic and Normative Systems. College Publications.

Gilboa, O.; Slawnych, N.; \& Palider, K. (forthcoming). Gender Differences and Biology. In Barseghyan, Patton, \& Shaw (Eds.) (forthcoming).

Hilpinen, R. \& McNamara, P. (2013). Deontic Logic: A Historical Survey and Introduction. In Gabbay et al. (Eds.) (2013), pp. 3-136.

Kaiser, D. (2005). Drawing Theories Apart: The Dispersion of Feynman Diagrams in Postwar Physics. University of Chicago Press.

Lambek, J. \& Scott, P.J. (1986). Introduction to Higher Order Categorical Logic. Cambridge University Press.

Le Novère, N.; Hucka, M.; Mi, H.; Moodie, S.; Schreiber, F.; Sorokin, A.; Demir, E.; Wegner, K.; Aladjem, M. I.; Wimalaratne, S. M.; Bergman, FT.; Gauges, R.; Ghazal, P.; Kawaji, H.; Li, L.; Matsuoka, Y.; Villéger, A.; Boyd, S. E.; Calzone, L.; Courtot, M.; Dogrusoz, U.; Freeman, T. C.; Funahashi, A.; Ghosh, S.; Jouraku, A.; Kim, S.; Kolpakov, F.; Luna, A.; Sahle, S.; Schmidt, E.; Watterson, S.; Wu, G.; Goryanin, I.; Kell, D. B.; Sander, C.; Sauro, H.; Snoep, J. L.; Kohn, K.; \& Kitano, H. (2009). The Systems Biology Graphical Notation. Nature Biotechnology 27 (8), pp. $735-741$.

Levesley, N. \& Barseghyan, H. (forthcoming). Diagramming Late-18th Century Chemistry. In Barseghyan, Patton, \& Shaw (Eds.) (forthcoming).

Loiselle, M. (2017). Multiple Authority Delegation in Art Authentication. Scientonomy 1, pp. 41-53. Retrieved from https://www.scientojournal.com/index.php/scientonomy/article/view/28233.

Mercuri, M. \& Barseghyan, H. (2019). Method Hierarchies in Clinical Epidemiology. Scientonomy 3, pp. 45-61. Retrieved from https://scientojournal.com/index.php/scientonomy/article/view/33559.

Overgaard, N. \& Loiselle, M. (2016). Authority Delegation. Scientonomy 1, pp. 11-18. Retrieved from https://scientojournal.com/index.php/scientonomy/article/view/27065.

Overgaard, N. (2017). A Taxonomy for the Social Agents of Scientific Change. Scientonomy 1, pp. 55-62. Retrieved from https://scientojournal.com/index.php/scientonomy/article/view/28234.

Palider, K. (2019). Reasons in the Scientonomic Ontology. Scientonomy 3, pp. 15-31. Retrieved from https://scientojournal.com/index.php/scientonomy/article/view/33557.

Patton, P.; Overgaard, N.; \& Barseghyan, H. (2017). Reformulating the Second Law. Scientonomy 1, pp. 29-39. Retrieved from https://www.scientojournal.com/index.php/scientonomy/article/view/27158.

Patton, P. (2019). Epistemic Tools and Epistemic Agents in Scientonomy. Scientonomy 3, pp. 63-89. Retrieved from https://scientojournal.com/index.php/scientonomy/article/view/33621.

Rapson, J. \& Shaw, J. (forthcoming). Diagramming 20th Century Aryan Physics. In Barseghyan, Patton, \& Shaw (Eds.) (forthcoming).

Rawleigh, W. (2018). The Status of Questions in the Ontology of Scientific Change. Scientonomy 2, pp. 1-12. Retrieved from https://scientojournal.com/index.php/scientonomy/article/view/29651.

Sarkar, A.; Blackwell, A. F.; Jamnik, M.; \& Spott, M. (2015). Interaction with Uncertainty in Visualisations. Eurographics Conference on Visualization (EuroVis) 2015, 133-137.

Sarwar, A. \& Patton, P. (forthcoming). Diagramming 19th Century Studies of Localization of Function in the Cerebral Cortex. In Barseghyan, Patton, \& Shaw (Eds.) (forthcoming).

Seidl, M.; Brandsteidl, M.; Huemer, C.; \& Kappel. G. (2012). UML @ Classroom. Springer.

Venn, J. (1880). On the Diagrammatic and Mechanical Representation of Propositions and Reasonings. The London, Edinburgh, and Dublin Philosophical Magazine and Journal of Science 9, pp. 1-18.

Zulfiqar, A. \& Patton, P. (forthcoming). Medieval Islamic Medicine. In Barseghyan, Patton, \& Shaw (Eds.) (forthcoming). 